Pure and Applied Mathematics Quarterly

Volume 8, Number 2

(Special Issue: In honor of

F. Thomas Farrell and Lowell E. Jones, Part 2 of 2)

$329-421,2012$

\title{
Controlled K-theory I: Basic Theory
}

\author{
Frank Quinn
}

\begin{abstract}
This paper provides a full controlled version of algebraic $K$-theory. There is a rich array of assembly maps; the controlled assembly isomorphism theorem identifying the controlled group with homology; and a statement of the stability theorem describing the behavior of the inverse limit as the control parameter goes to 0 . There is also a careful treatment of spectral cosheaf homology and related tools, including an "iterated homology identity" giving a spectrumlevel version of the Leray-Serre spectral sequence.
\end{abstract}

Keywords: algebraic K-theory, controlled algebra, controlled topology.

\section{INTRODUCTION}

Controlled algebra was originally developed to encode obstructions in controlled topology [Quinn 1, 2]. At that time attempts to develop controlled algebraic $K$-theory were unsuccessful, and the topological obstructions were instead formulated using pseudoisotopy. For many applications this is satisfactory, cf. [Quinn 2, 5, 7], [Hughes] but there are drawbacks: genuine $K$-theory is needed for a full controlled version of surgery; and computations using the assembly map structure are greatly enhanced by access to the full range of $K$-theory tools. For

Received January 17, 2007.

Partially supported by the National Science Foundation. 
example the remarkable work of Farrell and Jones and their coworkers [FarrellJones 1, 2] [Berklove-Farrell-Juan-Pineda-Pearson], [Davis-Lück], [Roushon], [Aravinda-Farrell-Roushon] is done in the context of pseudoisotopies in order to use the controlled pseudoisotopy theory developed in [Quinn 2]. From this they deduce information about Whitehead and lower $K$ groups of integral group rings. Controlled algebraic $K$-theory is needed to extend these results to higher $K$ groups and other coefficient rings. Recently [Bartels-Reich] have also made a start in this direction using continuously controlled $K$-theory. The continuous theory describes the inverse limit of finite- $\epsilon$ sets, but it is missing the "stability" theorem that describes how they approach this limit. The stability theorem is a crucial ingredient of many applications, c.f. [Quinn 2, 8].

This paper provides a version of controlled $K$-theory with most of the features that made the pseudoisotopy version successful so it should substitute for pseudoisotopy in many of the proofs of Farrell-Jones et. al. It also makes purely algebraic tools available. For example this theory and Dress induction [Dress] are used in [Quinn 8] to show that virtually abelian groups satisfy a refinement of the Farrell-Jones fibered isomorphism conjecture.

Most of the paper is detailed and self-contained, including even a description of sign conventions in chain complexes. The reason is that some of the proofs are quite delicate and don't work with the standard versions of $K$-theory. It is not a priori clear that modifications needed to get one thing to work won't wreck something else. For instance we use simplicial complexes of chain complexes. Vertices are complexes, edges are chain maps, and higher simplices are diagrams of chain maps. However requiring these diagrams to chain homotopy commute makes the theory too rigid. A weaker requirement on diagonals gives a functional theory but then it is not clear that it still gives $K$-theory. This is not a robust argument: a sign error or partial-order mistake could wreck the whole thing. It may be worth mentioning that it took the author 25 years to get it all to mesh properly. In any case a detailed and explicit treatment is appropriate.

There are significant overlaps with other material, particularly the work of [Igusa-Klein 1] and [Igusa 1] on higher Reidemeister torsion. Unfortunately there are enough differences in detail and in setting up $\epsilon$ control that it is quicker and safer to do it all from scratch. 
Section 2 contains statements of results, with sufficient explanation to make them usable without extensive reference to the body of the paper. The primary result in Part I is the Controlled assembly isomorphism theorem 2.2.1. A version of stability is included as Statement 2.3.1.

The body of the paper begins in Section 3 with a version of uncontrolled $K$ theory using chain complexes. Most of the section is occupied by the proof that this agrees with the Quillen definition. Section 4 adds control to the definitions of $\S 3$ and develops the elementary properties of the controlled $K$-space. In Section 5 deeper properties (those requiring spacial localization techniques) are developed. In particular the homology axioms needed in the following section are verified. Section 6 gives a development and axiomatic characterization of spectral cosheaf homology and assembly maps. The Controlled Assembly Isomorphism theorem follows from this because controlled $K$-theory satisfies the axioms.

\section{Results}

The central construction is a simplicial set of $\epsilon$-controlled chain complexes over a metric space, briefly described in 2.1. The first main result is the controlled assembly isomorphism theorem of 2.2. This describes inverse limits of controlled $K$-spaces as homology with spectral cosheaf coefficients. The stability theorem of 2.3 asserts that the inverse system approaches the limit in a nice way.

\subsection{Controlled $K$-theory spaces.}

Our central object is a simplicial set $K_{1}^{l f}(X ; p, R, \epsilon)$ defined given

(1) a locally compact metric space $X$,

(2) a map $p: E \rightarrow X$,

(3) a ring $R$, and

(4) a real number $\epsilon>0$.

This space is defined in $\S 4$ using controlled geometric chain complexes. Very roughly these are 2 -complexes decorated with $R$ data, mapping into $E$. The vertices of the 2-complex correspond to $R$-modules, the edges are homomorphisms between the modules, and 2-cells encode relations among the morphisms. These are "finitely generated" in the sense that the map of the 2-complexes into $X$ are proper (thus locally finite), and "controlled" in the sense that each cell has size 
less than $\epsilon$ in $X$. Straightforward properties needed in formulating the results are given in 2.1.1-2.1.3. Inverse limits are described in 2.1.4-2.1.5.

2.1.1 Naturality. A morphism between maps $(E \stackrel{p}{\rightarrow} X) \rightarrow(F \stackrel{q}{\rightarrow} Y)$ is a commutative diagram

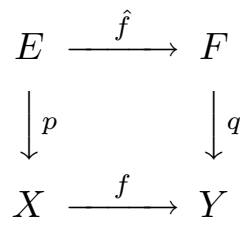

When $d(x, y)<\epsilon_{1}$ implies $d(f(x), f(y))<\epsilon_{2}$ this induces a map

$$
K_{1}^{l f}\left(X ; p, R, \epsilon_{1}\right) \rightarrow K_{1}^{l f}\left(Y ; q, R, \epsilon_{2}\right)
$$

defined simply by applying $\hat{f}$ to decorated 2-complexes mapping into $E$. These maps compose nicely, so define a functor on an appropriate catgory.

2.1.2 Restriction. If $U \subset X$ is open then there is a restriction map

$$
K_{1}^{l f}(X ; p, R, \epsilon) \rightarrow K_{1}^{l f}(U ; p \mid U, R, \epsilon) .
$$

In terms of decorated 2-complexes in $E$ this is given by restricting to the largest subcomplex mapping into $p^{-1}(U)$. Getting this to make sense near the edge of $U$ requires a wrinkle in the definition that will be appreciated by experts, see $\S 4.1 .7$.

2.1.3 Other properties. We mention several other basic aspects that will be explained in more detail when they are needed.

The first such aspect is that the path components of the space are essentially the $\epsilon-K_{1}$ obstructions arising from controlled h-cobordisms in [Quinn 1, 2]. This gives the connection to topology and also motivates the notation for the space.

Another basic topic is the use of control functions $\epsilon: X \rightarrow(0, \infty)$ rather than just constant $\epsilon>0$. This only makes a difference when $X$ is noncompact, but is almost always required in topological applications with noncompact $X$. The reasons we stick with constants here are: (1) details are simpler; (2) the function case follows from a relative form of the compact constant case by patching; and 
(3) the constant- $\epsilon$ conclusions for noncompact $X$ are stronger than the function- $\epsilon$ version, and the difference should be useful in geometric applications.

Finally, the role of the space $E$ is to specify "local fundamental groups" over $X$. If $E \rightarrow E^{\prime}$ is a map of spaces over $X$ that (roughly) is an isomorphism of fundamental groups of inverse images of points in $X$, then the induced map of $K_{1}$ spaces is (roughly) an equivalence, see 4.3.4. This is a consequence of the use of 2-complexes in the definition. This invariance property is very useful in applications but plays a minor role here.

2.1.4 The inverse limit. Define

$$
K_{1}^{l f}(X ; p, R)=\operatorname{holim}_{\epsilon \rightarrow 0} K_{1}^{l f}(X ; p, R, \epsilon) .
$$

The homotopy inverse limit is the space of paths to $\infty$ in the system. More precisely we define an inverse system indexed on positive integers, with the $n^{\text {th }}$ space the inverval $[n, \infty)$ and maps the inclusions. Then the homotopy inverse limit is the space of cofinal maps of inverse systems from this into the $K_{1}^{l f}(X ; p, R, *)$ system.

The on-the-nose (i.e. not homotopy) inverse limit is the intersection

$$
\cap_{\epsilon} K_{1}^{l f}(X ; p, R, \epsilon)=K_{1}^{l f}(X ; p, R, 0)
$$

and is uninteresting. The homotopy version loosens this up by allowing elements at different scales to be "homotopic" rather than equal. Behavior of the inverse system as it approaches the limit is desribed in $\S 2.4$.

2.2 Controlled assembly. This theorem uses the "spectral cosheaf homology" described in $\S 8$ of [Quinn 2] and in more detail in $\S 6$. The basic idea is that a continuous spectrum-valued functor of spaces can be applied to point inverses of a map $E \rightarrow X$ to get a "spectral cosheaf" over $X$. Homology with coefficients in such a thing can be defined and satisfies appropriate versions of the usual properties of homology. We think of homology as accessible, at least more so than $K$-theory, so a homological description is good news. A version for compactlysupported homology is given in 2.2.4.

2.2.1 Controlled assembly isomorphism theorem. Suppose $X$ is a locally compact metric space, $p: E \rightarrow X$ a map. Then

(1) (metric independence) up to homotopy $K_{1}^{l f}(X, p, R)$ is independent of the metric on $X$. 
(2) (spectrum structure) $K_{1}^{l f}(X, p, R)$ has a natural $\Omega$-spectrum structure. Denote the first deloop of this structure by $\mathbb{K}^{l f}(X ; p, R)$.

(3) (coefficients) If $E$ is a connected space with the homotopy type of a $C W$ complex then choice of basepoint $* \in E$ gives an equivalence of spectra with the (nonconnective) algebraic K-theory spectrum

$$
\mathbb{K}(\mathrm{pt} ; E \rightarrow \mathrm{pt}, R) \stackrel{\simeq}{\longrightarrow} \mathbb{K}\left(R\left[\pi_{1}(E, *)\right]\right) .
$$

(4) (assembly isomorphism) If $p$ is a stratified system of fibrations and $X$ is an ANR then the assembly map associated to the spectrum structure is an equivalence of spectra,

$$
\mathbb{H}^{l f}(X ; \mathbb{K}(p, R)) \stackrel{\simeq}{\longrightarrow} \mathbb{K}^{l f}(X ; p, R) .
$$

\subsubsection{Notes.}

(1) Stratified systems of fibrations are defined in [Quinn 2] and described in 6.2.1. The homotopy stratified group actions developed in [Quinn 5] naturally give rise to these in profusion.

(2) In (4) $\mathbb{K}(p, R)$ denotes the spectral cosheaf over $X$ obtained by applying $\mathbb{K}$ fiberwise to $p: E \rightarrow X$. This process is described in detail in $\S 6.7-6.8$.

(3) Statement (3) identifies the fibers in this cosheaf with standard $K$-theory of the fundamental group of the point inverses of $p$. The proof is unexpectedly delicate, and is given in $§ 3.6-3.10$.

(4) Statements (2)-(4) asserts that this functor is "self-computing" in the sense that it supplies both the spectrum needed to define the homology and the assembly map that makes the comparison. The isomorphism statement (4) is proved by by verifying that $\mathbb{K}^{l f}(X ; p, R)$ satisfies axioms that characterize homology. Stratified homology and the axioms are developed in $\S 6$. The elementary axioms are verified in $\S 4$, more difficult ones in $\S 5$.

(5) Metric invariance (1) is included in the isomorphism statement (4) because homology has this property. It seems striking enough to deserve a separate statement. Compare $R^{n}$ with its standard metric to the metric induced by a homeomorphism with the open unit ball. The first metric 
is complete so the typical $K_{1}$ gadget is infinite, has $\delta$-dense image, and satisfies the relations everywhere. The second metric is not complete, and in fact the complement of any open neighborhood of the metric frontier (see 2.1.2) is compact. This means $K_{1}$ gadgets are finite and there is a big "ragged edge" near the metric frontier where structure relations fail. Nonetheless in the limit the "ragged edge" shrinks away, and the map from the complete metric to the incomplete one does not lose information. Metric invariance is proved in $\S 5.4$.

2.2.3 Compactly supported homology. Ordinary homology can be obtained as the direct limit of homology of finite complexes mapping into the space. Since finite complexes are compact, "locally finite" and ordinary homology coincide, so we define

$$
K_{1}(X ; p, R)=\operatorname{holim}_{h: Y \rightarrow X} K_{1}^{l f}\left(Y ; h^{*} p, R\right),
$$

where the limit is over the system of finite complex $Y \rightarrow X$, and $h^{*} p: h^{*} E \rightarrow Y$ denotes the pullback (fiber product) of $p$ along $h$. Morphisms in the inverse system are maps of finite complexes commuting with the maps to $X$.

This version is defined for all $X, p$ (no local compactness requirement) and is functorial with respect to all morphisms. The locally finite theorem 2.2.1 gives, by direct limits:

Theorem. Suppose $X$ is a metric space and $p: E \rightarrow X$ is a stratified system of fibrations. Then the limit $K_{1}(X ; p, R)$ has a natural spectrum structure, and the assembly map

$$
\mathbb{H}(X ; \mathbb{K}(p, R)) \stackrel{\simeq}{\longrightarrow} \mathbb{K}(X ; p, R) .
$$

is an equivalence of spectra.

2.3 Stability. The $K$-space $K_{1}^{l f}(X ; p, R)$ is defined to be the homotopy inverse limit of spaces $K_{1}^{l f}(X ; p, R, \epsilon)$, as $\epsilon \rightarrow 0$. "Stability" makes properties of the limit available at finite- $\epsilon$ scales by showing the inverse system converges nicely. The version given here is sufficient for most applications. Note, however, that a proof is not included; see the discussion following the statement.

To get a non-compact statement we extend the definition of $K_{1}^{l f}(X ; p, R, \epsilon)$ in the evident way to permit $\epsilon$ to be a continuous function $X \rightarrow(0, \infty)$. See 2.1.3 for a brief discussion of control functions. 
2.3.1 Statement. Suppose $X$ is a finite dimensional locally compact metric ANR, $p: E \rightarrow X$ is a stratified system of fibrations, and $\epsilon: X \rightarrow(0, \infty)$ is given. Then there is $\delta: X \rightarrow(0, \infty)$ so that the relax-control map $\delta \rightarrow \epsilon$ homotopically factors through the limit in the sense that there is a map $S$ so that the diagram

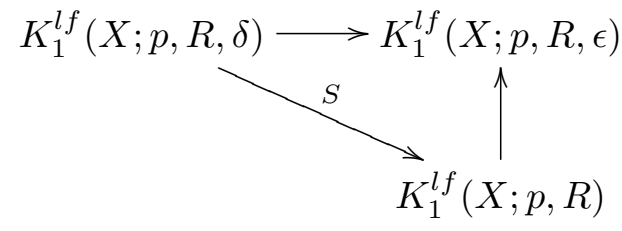

homotopy commutes.

This is a key result: applications that require it include the controlled $h-$ cobordism theorem [Quinn 1, 2]; the results of Farrell-Hsiang on $K$-theory of Bieberbach groups, extended to virtually-abelian groups in [Quinn 8]; and the asymptotic transfer of [Farrell-Jones 2]. Moreover this paper could be simplified considerably if stability were not one of the goals: there are categorical formulations of the inverse limit space, c.f. [Pedersen], that allow use of category-oriented work of Quillen and many others. The proof of stability, in contrast, seems to require careful and explicit work at the simplex level. This explicit work occupies much of $\S 5$ and preparation for it occupies much of the earlier sections.

This paper does not include a proof of stability. The lemmas of $\S 5$ are designed to plug in the proof of stability for pseudoisotopy in [Quinn 2], so a proof could be obtained that way. This can be simplified, for instance by using ideas in the proof of stability for surgery groups in [Pedersen-Yamasaki]. However stronger results are needed: Farrell and Jones, for instance, needed a version with "foliated control", see [Bartels-Farrell-Jones-Reich]. The old proof can probably be modified to provide some foliated control, but instead I am developing a different approach that seems likely to be much simpler and may give significantly sharper results. This will be described in a later paper.

\section{Chain complexes And $K_{1}(R)$}

A space $K_{1}(R)$ is defined as a simplicial set whose simplices are diagrams of chain complexes satisfying various conditions. The classical $K_{1}$ group is $\pi_{0}$ of this space. 
As mentioned in $\S 1$ this material overlaps work of [Igusa] and [Igusa-Klein]. Myriad delicate differences in detail and their use of categorical techniques prevents us from building directly on their work.

Chain notations, suspensions, and mapping cones are described in 3.1, Triangular simplices are defined in 3.4. $K_{1}(R)$ is finally defined in 3.5. One of the main technical tools, the cancellation of inverses, makes its first appearence in 3.6. In 3.7 we give the first application: a deformation retraction of the $K_{1}$ space to a subcomplex equivalent to the Volodin description of $K$-theory.

3.1 Chain complexes. This section mainly fixes notation and sign conventions. Our sign conventions differ from some others, and this is explained in 3.1.5.

3.1.1 Notation. To simplify the formulae we use a uniform notation for the boundary homomorphisms and chain maps in the structure. For example a chain complex will have the form $(C, c)$ where $C$ are the chain groups and $c: C \rightarrow C$ denotes the boundary homomorphism. Lower indices will be used to indicate different complexes, and upper indices degrees in the complex. For instance $C_{i}^{j}$ indicates the degree- $j$ group of the chain complex $C_{i}$.

3.1.2 Simplices of complexes. Elaborating on 3.1.1, an $n$-simplex of complexes consists of chain complexes $\left(C_{i}, c_{i}\right)$ for $i=0, \ldots, n$ and for $i<j$, chain maps $c_{i, j}: C_{i} \rightarrow C_{j}$.

Note that this definition does not require any compatibility (e. g. homotopy commutivity) for the chain maps. Compatibility conditions will be added later.

The face $\partial_{i}$ of a simplex is defined by omitting the $i^{\text {th }}$ vertex. An $(n-1)$ simplex is supposed to be indexed on the set of integers $[0, n-1]$, so more precisely $\partial_{i}$ is obtained from the order-preserving injection $[0, n-1] \rightarrow[0, n]$ onto the complement of $i$.

More generally if $J \subset[0, n]$ we define $\partial_{J}$ by composing with the orderpreserving injection $[0, n-|J|] \rightarrow[0, n]$ whose image is the complement of $J$. If we denote the injection by $\alpha$ then

$$
\left(\partial_{J}(C, c)\right)_{i}=C_{\alpha(i)}
$$

This degree of precision in the notation is rarely needed. 
3.1.3 Suspensions. The suspension of a $Z$-graded module $C$ is denoted by $S C$, and is the same module with grading shifted up by one. Explicitly, $(S C)^{r}=$ $C^{r-1}$. Thus for instance the identity map $S C \rightarrow C$ has degree -1 .

The suspension of an $n$-simplex of complexes $(C, c)$ has graded module the suspension $S C$ and structure maps with signs according to the parity of their degree. Boundaries have degree 1 and chain maps have degree 0 so

$$
\begin{aligned}
s c_{i} & =(-1) c_{i} \\
s c_{i, j} & =(+1) c_{i, j}
\end{aligned}
$$

See 3.1.5 for comments on this choice of sign conventions.

3.1.4 Mapping cones. Mapping cones of chain isomorphisms will be used to add trivial summands in $K$-theory.

Suppose $f: C \rightarrow \bar{C}$ is a chain map. Then the cone $\bar{C} \oplus_{f} S C$ is the complex with graded modules $\bar{C} \oplus S C$, and boundary homomorphisms $\left(\begin{array}{cc}\bar{c} & f \\ 0 & -c\end{array}\right)$.

Lemma. If $f$ is an isomorphism then $\bar{C} \oplus_{f} S C$ is contractible with canonical contraction $\left(\begin{array}{cc}0 & 0 \\ f^{-1} & 0\end{array}\right)$

Contractibility of the cone requires only that $f$ be a chain homotopy equivalence, though in that case the contraction depends on the chain homotopies as well as the map itself. However the isomorphism case is the one needed here.

3.1.5 Sign conventions. Sign adjustments are required in suspensions and cones to get the various identities to work out. There are two standard choices. The one used here has signs in the suspension structure: odd degree structure morphisms (boundary homomorphisms and homotopies) pick up a -1 upon suspension. Suspensions of chain maps are unchanged because they have degree 0 . And if $f: C \rightarrow D$ is a degree-0 chain map, then the same homomorphism is a degree- $(-1)$ chain map $S C \rightarrow D$. The different sign requirements for the different degrees (see 3.1.3) comes from the signs in the suspension.

The other convention does not change signs in suspensions, but then signs are required in morphisms. If $f: C \rightarrow D$ is a degree-0 chain map then to get a degree-(-1) chain map $S C \rightarrow D$ one uses $(-1)^{n} f^{n}$ on the degree- $n$ summand of $S C$. This is the convention used by Ranicki, for instance. The notation in this 
convention is more elaborate because it explicitly uses the grading in $C$ rather than the degree of the map.

Topologically these conventions correspond to different orders in a product. If the suspension of $\Delta^{n}$ is taken to be $I \times \Delta^{n}$ then the usual product sign conventions give the signed suspension used here. If the suspension is taken to be $\Delta^{n} \times I$ then suspensions don't get signs, but morphisms do.

3.2 Triangular morphisms and simplices. Diagonal, triangular and increasing morphisms of based modules are defined in 3.2.1-3. In 3.3.4 diagonal morphisms on infinitely generated modules are shown to be well-behaved if the partial orders are "locally bounded".

3.2.1 Based modules. As usual, a basis for a module is a set of generators as a free module over the ring. In fact a basis renders the algebraic structure of the module itself irrelevant, but for the time being we retain the language as a comfortable context.

If $C \supset D$ is a based submodule (i.e. generated by a subset of the basis) then $C \perp D$ is the perpendicular submodule, generated by the rest of the basis. There is a canonical sum decomposition $C=D \oplus(C \perp D)$, and isomorphism $C \perp D \simeq C / D$. In controlled situations quotient modules behave badly, and perpendicular submodules will be used instead. Finally if $f: C \rightarrow \bar{C}$ is a homomorphism of based modules and $C \supset D, \bar{C} \supset \bar{D}$ are based submodules, then $f$ induces homomorphisms $D \rightarrow \bar{D}$ and $C \perp D \rightarrow \bar{C} \perp \bar{D}$. If we represent $f$ as a matrix with respect to the bases then these induced homomorphisms are obtained by taking appropriate submatrices. Algebraically they are described as compositions with the canonical inclusions and projections associated to the sum decomopositions.

3.2.2 Diagonal homomorphisms. Suppose $C, D$ are free modules with specified bases denoted by base $(C)$, base $(D)$ respectively. The support, $\operatorname{supp}(f)$, of a homomorphism $f: C \rightarrow D$ is the subset of the basis of $C$ on which $f$ is nontrivial. The homomorphism is diagonal if is induced by an injection base $(f): \operatorname{supp}(f) \rightarrow$ base $(D)$.

More generally, if $U$ is a subset of $R$ then $f$ is $U$-diagonal if there is a diagonal map base $(f)$ so that for each basis element $x \in \operatorname{base}(C), f(x)$ is a multiple of base $(f)(x)$ by an element of the subset $U$. In these terms "diagonal" is the 
same as $\{1\}$-diagonal. Other useful cases are $U=\{ \pm 1\}$, and $U$ the units of $R$. In topology one often has a group $\operatorname{ring} R=Z[\pi]$, and $U= \pm \pi$. Completely arbitrary coefficients correspond to $R$-diagonal maps.

In the definition of "diagonal" we are extending the convention that the free module with empty basis is 0 . Namely, extend the basis function of $f$ to base $(C) \rightarrow$ base $(D) \cup\{\emptyset\}$ by taking all elements not in the support of $f$ to the empty set. Then we take as a convention that the only possible multiple of the empty basis element is 0 . These conventions simplify the language in several places. For example the composition of diagonal maps is diagonal, and with this convention if the coefficients are units then the basis function of the composition is the composition of basis functions.

Note that an $R$-diagonal homomorphism is an isomorphism if and only if the underlying basis function is a bijection of bases, and the coefficient on each basis element is a unit in the ring. In this case the inverse is evidently also diagonal, with inverse base function.

3.2.3 Triangular homorphisms. Fix a subgroup $U$ of the units of $R$. Suppose $f: C \rightarrow D$ is a homomorphism of free based $R$-modules with partial orders on the bases. $f$ is $U$-triangular with respect to the partial orders if there is a decomposition $f=h+u$ where

(1) $h$ is $U$-diagonal;

(2) base $(h): \operatorname{supp}(h) \rightarrow$ base $(D)$ is order-preserving; and

(3) $u$ is increasing with respect to $h$ in the sense that for each basis element $x \in C, u(x)$ lies in the submodule of $D$ generated by basis elements strictly greater than base $(h)(x)$ in the partial order.

With appropriate hypotheses (see 3.2.4) the decomposition is unique. In this case the diagonal part is denoted by $\operatorname{diag}(f)$. We are using the convention that base $(h)(x)=\emptyset$ if $x$ is not in the support of $h$. In this case there are no greater basis elements and so $u(x)=0$. In other words the support of $u$ is contained in the support of $h$.

Note that the increasing condition in (3) only uses the partial order in base $(D)$, and in particular does not depend on the underlying base function of $h$ being order-preserving. Both conditions are needed to show that composition of triangular functions is triangular. 
When the domain and range are the same there is a natural notion of "increasing": $u(x)$ lies in the submodule generated by basis elements strictly larger than $x$. In the terminology above this is the same as "increasing with respect to the identity", and is equivalent to requiring $1+u$ to be triangular.

We explain why we use the term "increasing" instead of the more common "upper triangular" or "lower triangular". The usual practice is to refine the partial order to a total order, and then write the morphism as a matrix. We think of elements of the modules as column vectors, with morphisms acting as matrices on the right. "Diagonal" entries then occur in blocks along the diagonal (and can usually be arranged to be exactly on the diagonal). "Increasing" entries lie below this (block) diagonal. There are two problems. First, we make extensive use of matrix notations, but make no attempt to relate the implicit order in the matrix to the structural partial orders. Thus the matrices often have entries on both sides of the diagonal. Second it seems to invite confusion to try to use "lower" as a synonym for "increasing".

3.2.4 Locally bounded partial orders. This is a condition to ensure that triangular morphisms of infinitely generated modules are well-behaved. It is automatically satisfied for finitely-generated modules, and we use it here to simplify definitions. In controlled situations definitions this condition may not hold and we will have to explicitly assume some of its consequences in the definitions.

A partial order is locally bounded if there is no element that is the starting point of arbitrarily long increasing chains. We say a module is locally bounded if the partial order on the basis is locally bounded. The following is standard:

Lemma. Homomorphisms in the following are understood to be triangular homomorphisms of modules with locally bounded partially ordered bases.

(1) the diagonal-plus-increasing decomposition is well-defined, and is denoted by $\operatorname{diag}(f)+\operatorname{inc}(f)$;

(2) the diagonal part is functorial: $\operatorname{diag}(f g)=\operatorname{diag}(f) \operatorname{diag}(g)$,

(3) if $f-g$ is increasing with respect to the diagonal part of one of $f, g$, then $\operatorname{diag}(f)=\operatorname{diag}(g)$,

(4) A diagonal-plus-increasing homomorphism is an isomorphism if and only if the diagonal part is an isomorphism. This is equivalent to the basis function being bijective and the coefficients being units; 
(5) the inverse of a triangular isomorphism is triangular.

(6) there is a canonical (independent of the partial order) factorization $f=$ $\ldots\left(1+\alpha_{n}\right) \ldots\left(1+\alpha_{1}\right) \operatorname{diag}(f)$, locally finite, with $\alpha_{i}$ increasing, and $\alpha_{i} \alpha_{j}=0$ if $j \leq i$.

Proof. Suppose $f: C \rightarrow D$ is triangular. To see (1), define a decreasing filtration on the basis of $D$ with $n^{\text {th }}$ subset the elements that are the smallest in an increasing chain of length at least $n$. Let $D_{n}$ be the submodule generated by the $n^{t h}$ subset. Then $D_{0}=D$, and $\cap_{n} D_{n}=0$ by the locally bounded condition. The increasing part of any triangular map must have image in $D_{1}$, so $f: C \rightarrow D_{0} / D_{1}$ is induced by an injection on part of the basis and takes the rest to 0 . This determines $h$ on the part of the basis that does not map into $D_{1}$, and thus also $u$ on this subset. Restrict to the complement, then we have $f_{1}: E_{1} \rightarrow D_{1}$ triangular. The same argument determines the decomposition on the part of the basis that does not map into $D_{2}$. Continuing by induction shows the entire decomposition is determined.

For statement (2) let $f=h+u, g=j+v$ denote the decompositions. Then $f g=h j+(h v+u j+u v) . h j$ is diagonal, and since $h, j$ are order-preserving and $u, v$ are increasing, the other terms are increasing. This gives a diagonal-plusincreasing decomposition of $f g$.

Statement (3) should be clear.

For (4) it is easy to see that if the diagonal part is not an isomorphism then the whole homomorphism cannot be either. Conversely suppose the diagonal part is an isomorphism. Denote the decomposition by $h+u$, then the inverse is given by

$$
(h+u)^{-1}=\Sigma_{i=0}^{\infty}\left(-h^{-1} u\right)^{i} h^{-1} .
$$

The infinite sum is well-defined because it is finite when applied to any element of $D$. This follows from the bounded hypothesis by essentially the argument used to show (1). The form of the inverse gives (5). For the second statement in (4) a diagonal morphism decomposes as a direct sum of a zero part, and a sum of endomorphisms of $R$ indexed by the underlying basis function. To be an isomorphism the basis function must be a bijection, and each 1-dimensional summand must be an isomorphism. The latter condition is that the coefficients must be units in the ring. 
Finally for $(6)$ we let $\widehat{\operatorname{diag} f} f$ denote $(\operatorname{diag} f)$ as a homomorphism from the span of elements on which it is nonzero, to the span of the image of those elements. This is invertible so the inverse is defined, and the support property mentioned above implies that $f=f(\widehat{\operatorname{diag} f})^{-1} \operatorname{diag} f$. The composition $f(\widehat{\operatorname{diag} f})^{-1}$ is of the form $p+u$, with $p$ a projection on a subset of the basis and $u$ increasing. $p+u=(1+u) p$ so it is sufficient to factor $1+u$.

We factor $1+u$ using essentially the opposite of the argument for (1). Let $D_{0} \subset D_{1} \subset \cdots$ be a maximal chain of based submodules so that $D_{0}=0$ and $u\left(D_{i}\right) \subset D_{i-1}$ for $i>0$. Since $u$ is increasing and the order is bounded this sequence terminates locally. Restrict to a set where it terminates at level $n$. Let $p_{i}$ denote the based projection $D \rightarrow D_{i}$. Inductively define $\alpha_{i}$ by $\alpha_{0}=0$ and

$$
\alpha_{i}=\left(u-\Sigma_{j=0}^{i-1} \alpha_{j}\right) p_{i} .
$$

It follows that $\alpha_{i} p_{i}=\alpha_{i}$ (definition); $\alpha_{i} p_{j}=0$ for $j<i$ (induction); $p_{i-1} \alpha_{i}=\alpha_{i}$ (definition of $D_{i}$ ); and $u=\sum_{j=0}^{n} \alpha_{j}$ (since $D_{n}=D$ ). From these it follows that $\alpha_{i} \alpha_{j}=0$ if $j \leq i$. Thus

$$
\left(1+\alpha_{n}\right) \cdots\left(1+\alpha_{2}\right)\left(1+\alpha_{1}\right)=1+\sum_{j=0}^{n} \alpha_{j}=1+u .
$$

3.3 Contractions and cancellation. We use chain contractions to identify parts of a complex that can safely be omitted. Lemma 3.3.2 shows this is the inverse of the usual stabilization operation, but with more flexibility in how the trivial summands are related to the chosen bases. An explanation of the topological significance is given in 3.3.3.

3.3.1 Definition. Suppose $C$ is a based complex with a partial order on the basis in each degree, and $\xi$ is a chain contraction for $C$. We say $\xi$ cancels the complement of a based subcomplex $\hat{C} \subset C$ if

(1) in each degree and when they are comparable, basis elements of $C \perp \hat{C}$ preceed those of $\hat{C}$ in the partial order, and

(2) there is a based decomposition $C \perp \hat{C}=D \oplus \bar{D}$ so that $\xi$ has the form

$$
\left(\begin{array}{lll}
\hat{\xi} & u & v \\
0 & 0 & \delta \\
0 & 0 & 0
\end{array}\right): \hat{C} \oplus D \oplus \bar{D} \longrightarrow \hat{C} \oplus D \oplus \bar{D}
$$

with $\delta$ a \pm 1-triangular isomorphism. 
This is a very rigid condition. If the partial order and $\xi$ are fixed then (1) implies that the based subcomplexes for which this works are linearly ordered by inclusion, and the decomposition in (2) is unique when it exists. The following is also clear:

3.3.2 Lemma. If $\xi$ cancels the complement of $\hat{C} \subset C$ then it preserves $\hat{C}$ and the restriction ( $\hat{\xi}$ in the matrix above) is a chain contraction of $\hat{C}$. If $\hat{B} \subset \hat{C} \subset C$ are based subcomplexes and $\xi$ cancels the complements in $C$ of both, then $\xi \mid \hat{C}$ cancels the complement of $\hat{B}$ in $\hat{C}$.

3.3.3 Structure of the boundary.

If $C=\hat{C} \oplus D \oplus \bar{D}$ is a cancellation decomposition as above then the boundary homomorphism $c: C \rightarrow C$ has the form

$$
\left(\begin{array}{ccc}
\hat{c} & x & y \\
0 & d & z \\
0 & \delta^{-1} & \bar{d}
\end{array}\right)
$$

The lower-left 0 entries reflect the hypothesis that $\hat{C}$ is a subcomplex. The $\delta^{-1}$ term comes from the hypothesis that $\xi$ is a chain contraction.

3.3.4 Standardizing cancellations. The following lemma shows that cancellations are equivalent to standard models. Versions of this are used to standardize dimensions in 3.8 and in the local cancellation of inverses in $\S 5$.

Lemma. Suppose $C=\hat{C} \oplus D \oplus \bar{D}$ is a cancellation decomposition of $C$ and use the notation above for the matrix form of the boundary homomorphism. Then $f$ is a chain isomorphism to the same graded module with boundary homomorphism $b$ and contraction $\beta$ where

$$
f=\left(\begin{array}{ccc}
1 & 0 & x \delta \\
0 & 1 & d \delta \\
0 & 0 & 1
\end{array}\right), \quad b=\left(\begin{array}{ccc}
\hat{c} & 0 & 0 \\
0 & 0 & 0 \\
0 & \delta^{-1} & 0
\end{array}\right), \quad \text { and } \quad \beta=\left(\begin{array}{ccc}
\hat{\xi} & u & -\hat{c} u \\
0 & 0 & \delta \\
0 & 0 & 0
\end{array}\right)
$$

Proof. The chain-map claim is that $f b=c f$, the contraction claim is that $f \beta=$ $\xi f$. Multiply out the matrices given, then this can be seen to follow from $c^{2}=0$. Alternatively, $b, \beta$ are obtained by conjugating by $f$ and $f^{-1}$ is obtained by multiplying the off-diagonal terms of $f$ by $-1 . f$ is \pm 1 triangular with respect to a suitable partial order because $\delta$ is. 
There are several useful remarks about this:

(1) The complement of $\hat{C}$ is also a subcomplex in this structure, so in particular the projection to $\hat{C}$ is a chain map.

(2) $f$ is determined by $\hat{C}$ and $\xi$, and in particular is independent of a choice of partial order. The submodule $D$ is the image of $\xi$ intersected with the complement of $\hat{C}$, so it's complement $\bar{D}$ is also determined. Let $p$ denote the based projection $C \rightarrow \bar{D}$, then $f=(1-p)+c p \xi$ is a canonical description.

(3) The contraction $\beta$ can be further improved. The degree- 2 homomorphism

$$
\left(\begin{array}{lll}
0 & 0 & u \\
0 & 0 & 0 \\
0 & 0 & 0
\end{array}\right)
$$

gives a chain homotopy to one with 0 in the upper right corner.

3.4 Definition of $K_{1}(R) . K_{1}(R)$ is a simplicial set with simplices defined using triangular simplices of chain complexes with contractions. The basic idea is that a $K_{1}$ simplex is a sum of a nondegenerate part, in which the chain maps are isomorphisms, and a cancellable part in which they may not be. Genuine $K$ theory activity takes place in the nondegenerate part, while the cancellations organized by the contractions enable stabilization of dimension. Most of the complexity of the theory comes from the cancellations: not from $K$-theory itself, but from the structures needed to keep it from escaping during stabilization.

3.4.1 Simplices in $K_{1}(R)$. An $n$-simplex of $K_{1}(R)$ is a finitely generated based chain $n$-simplex $C$ together with chain contractions $\xi_{i}$ for $\left(C_{i}, c_{i}\right)$, such that there are partial orders on the bases in each degree so that for each $i<j$,

(1) $c_{i, j}$ is a \pm 1 triangular chain map $C_{i} \rightarrow C_{j}$;

(2) the elements in the image of the basis function of $c_{i, j}$ come after the elements in the complement, in the partial order on each $C_{j}^{n}$;

(3) if $i<j<k$ then $c_{j, k} c_{i, j}$ has the same underlying basis function as $c_{i, k}$;

(4) $\xi$ cancels the complement of the image $C_{j} \perp c_{i, j}\left(C_{i}\right) \subset C_{j}$, for all $i$.

Note that the partial orders are only required to exist and are not part of the data. Diagrams of chain maps in the simplex, such as the triangle in (3), are not required to commute. However condition (3) is a weak version of "commute 
up to increasing chain homotopy". These conditions are delicately balanced in being strong enough to capture $K$-theory but flexible enough to work in the cancellation-of-inverses construction of the next section and various localizations of it. The following shows the images are based subcomplexes, which is required for condition (4) to make sense.

Lemma. The image of $c_{i, j}$ is the based subcomplex spanned by the image of the underlying basis function, and if $i<j<k$ then $c_{j, k} c_{i, j}$ has the same image as $c_{i, k}$.

The first part follows from the triangularity of $c_{i, j}$ and the order condition (2). The second part follows from this and the basis-function hypothesis (3).

Faces of such simplices are defined in the evident way: the $j^{\text {th }}$ face omits the complex $C_{j}$. Faces of $K_{1}$ simplices are again in $K_{1}$, so $K_{1}(R)$ is a simplicial complex. More precisely this gives it the structure of a $\Delta$-set (omit degeneracies from the definition of simplicial set, [Rourke-Sanderson 1]). One can either work with these as $\Delta$-sets or introduce degeneracies by introducing duplicates of the $C_{i}$. In any case we proceed without mention of degeneracies and leave the choice of details to the reader.

3.4.2 The image partial order. We expand on the partial order condition (2) in the definition of simplices in $K_{1}(R)$. Suppose $(C, c, \xi)$ is an $n$-simplex. Let $C_{i, j}$ denote the image of $c_{i, j}$ in $C_{j}$, and for notational convenience we set $C_{j, j}=C_{j}$. Recall that $C_{i+1, j}^{n} \perp C_{i, j}^{n}$ denotes the based complement of the smaller image in the larger, in degree $n$. Since in each degree the complement of $C_{i, j}$ preceeds it in the partial order we get

$$
\left(C_{j, j}^{n} \perp C_{j-1, j}^{n}\right)<\cdots<\left(C_{i, j}^{n} \perp C_{i-1, j}^{n}\right)<\cdots<\left(C_{1, j}^{n} \perp C_{0, j}^{n}\right)<C_{0, j}^{n} .
$$

We will refer to this as the "image partial order" on $C_{j}^{n}$. Any partial order making $(C, c, \xi)$ a $K_{1}$ simplex must refine this partial order.

3.4.3 Morphisms. Morphisms of $K_{1}$ simplices are dictated in a straightforward way by the structure. The key property is that triangulation gives $K_{1}$ simplices, so morphisms can be used to construct simplicial homotopies. Composition of $K_{1}$ morphisms is generally not a morphism, because the partial orders will usually not be compatible. 
A morphism of $K_{1}$ simplices $f:(A, \alpha) \rightarrow(C, \xi)$ consists of chain maps $f_{i, j}: A_{i} \rightarrow$ $C_{j}$ for $i \leq j$ such that there are choices of partial orders satisfying

(1) $(A, \alpha)$ and $(C, \xi)$ are $K_{1}$ simplices with respect to the partial orders;

(2) in each degree each $f_{i, j}$ is a \pm 1 triangular morphism whose image basis elements follow those in the complement;

(3) if $i \leq j \leq k$ then the morphisms $c_{j, k} f_{i, j}, f_{i, k}$, and $f_{j, k} a_{i, j}$ all have the same underlying set function; and

(4) $\xi_{j}$ cancels the complement of the image of $f_{i, j}$.

3.4.4 Triangulation. Suppose $f: A \rightarrow C$ is a morphism of $K_{1}$ simplices. We think of this as a diagram modeled on $\Delta^{n} \times I$, and triangulate it into $(n+1)$-simplices corresponding to the canonical triangulation of the model. Specifically we get $n+1$ such simplices, the $k$ th one of which has vertices $A_{0}, \ldots A_{k}, C_{k}, \ldots, C_{n}$, and chain maps the $a_{i, j}, c_{i, j}$ and $f_{i, j}$ whose domain and range are both vertices. $\partial_{0}$ of the 0 th simplex is $C, \partial_{n+1}$ of the $(n+1)$ st is $A$, and the $k$ th and $(k+1)$ th have exactly one face in common.

The following is straightforward:

Lemma. Simplices in the triangulation of a $K_{1}$ morphism are $K_{1}$ simplices.

We describe how this is used to construct homotopies. Suppose $X$ is a simplicial complex, and $A, C: X \rightarrow K_{1}(R)$ are simplicial maps. Suppose further that there are morphisms $f_{\sigma}: A(\sigma) \rightarrow C(\sigma)$ given for every $\sigma \in X$ so that $f_{\partial_{i} \sigma}=\partial_{i} f_{\sigma}$. Then simplices in the triangulations of the $f_{\sigma}$ fit together to define a homotopy from $A$ to $C$.

3.5 Cancellation of inverses. Direct sum defines a simplicial monoid structure $K_{1} \times K_{1} \rightarrow K_{1}$. "Simplicial" here means the product is defined on the simplicial product in which the set of $n$-simplices is the product of the sets of $n$-simplices of the factors. Alternatively this is a simplicial object in the category of monoids. Beware that we do not know if the geometric realization of the simplicial product is equivalent to the topological product of realizations. This would be the case if $K_{1}$ satisfied some version of the Kan condition, but this is far from true. In any case the use we make of the monoid structure is to construct various simplicial maps and homotopies that can be reliably realized. 
In this section we show the suspension is a homotopy inverse for the monoid structure by constructing a nullhomotopy of the sum id $\oplus S: K_{1}(R) \rightarrow K_{1}(R)$. This nullhomotopy is described in detail because elaborations of it are the bases for most of the results in the paper. The first application is in 3.7 to compress complexes into two degrees.

The construction is canonical, so it is sufficient to describe the effect on an arbitrary simplex of $K_{1}$ and they will fit together to define a homotopy of the entire space. The idea is that the chain contraction provides an isomorphism to the cone on the identity: if $(C, \xi)$ is an $n$-simplex then

$$
\left(\begin{array}{cc}
1 & -\xi \\
0 & 1
\end{array}\right): C \oplus S C \stackrel{\simeq}{\longrightarrow} C \oplus_{1} S C .
$$

The cone on the identity has an obvious global cancellation structure $(\bar{D}=C$, $D=S C)$ so $0 \rightarrow C \oplus_{1} S C$ is a morphism of $K_{1}$ simplices. Subdividing these two morphisms (see 3.4.4) gives a homotopy to 0. In detail:

3.5.1 Lemma. Suppose $(C, c, \xi)$ is an n-simplex of $K_{1}(R)$.

(1) $C \oplus S C$ with contraction $\left(\begin{array}{cc}\xi & 0 \\ 0 & -\xi\end{array}\right)$ is a $K_{1}$ simplex;

(2) the cone $C \oplus_{1} S C$ with contraction $\left(\begin{array}{ll}0 & 0 \\ 1 & 0\end{array}\right)$ is a $K_{1}$ simplex;

(3) $f_{i, j}=\left(\begin{array}{cc}1 & -\xi_{j} \\ 0 & 1\end{array}\right)\left(\begin{array}{cc}c_{i, j} & 0 \\ 0 & c_{i, j}\end{array}\right)$ for $i \leq j$ is a morphism between these simplices; and

(4) the inclusion $0 \rightarrow C \oplus_{1} S C$ is a $K_{1}$ morphism.

Proof. The main thing to check is that there are appropriate partial orders in which contractions cancel complements. Choose $K_{1}$-simplex partial orders on bases, and as in 3.4.2 denote the image of $c_{i, j}$ by $C_{i, j}$. Partial order the bases in the modules $C \oplus S C$ using the given partial order in each summand and by shuffling the image partial orders (3.4.2) in the two pieces, with $S C$ terms before $C$ terms. More specifically the degree $n$ module in $C_{j} \oplus S C_{j}$ is partially ordered as follows (recall $\left.\left(S C_{j}\right)^{n}=C_{j}^{n-1}\right)$ :

$$
\begin{aligned}
\left(C_{j, j}^{n-1} \perp C_{j-1, j}^{n-1}\right) & <\left(C_{j, j}^{n} \perp C_{j-1, j}^{n}\right)<\cdots \\
<\left(C_{i, j}^{n-1} \perp C_{i-1, j}^{n-1}\right) & <\left(C_{i, j}^{n} \perp C_{i-1, j}^{n}\right)<\cdots<C_{0, j}^{n-1}<C_{0, j}^{n} .
\end{aligned}
$$

$i \rightarrow j$ maps In both the sum and the cone the $i \rightarrow j$ maps are $\left(\begin{array}{cc}c_{i, j} & 0 \\ 0 & c_{i, j}\end{array}\right)$. These are \pm 1 triangular injections because $c_{i, j}$ is. The images are the based 
subcomplexes $C_{i, j} \oplus S C_{i, j}$ and satisfy the image order condition because the $c_{i, j}$ do. The $i \rightarrow j$ map in the morphism is the composition of $\left(\begin{array}{cc}c_{i, j} & 0 \\ 0 & c_{i, j}\end{array}\right)$ and $\left(\begin{array}{cc}1 & -\xi \\ 0 & 1\end{array}\right)$. The first factor is \pm 1 triangular as before. The second is a \pm 1 triangular isomorphism because $\xi$ preserves images, by Lemma 3.4.1, and $S C$ terms in the image partial order preceed $C$ terms. Specifically the summand $\left(C_{i, j}^{n-1} \perp C_{i-1, j}^{n-1}\right)$ is taken to $C_{i, j}^{n}=\oplus_{k \leq i}\left(C_{k, j}^{n} \perp C_{k-1, j}^{n}\right)$, and all parts of this are greater in the partial order. Thus the non-diagonal part of $\left(\begin{array}{cc}1 & -\xi \\ 0 & 1\end{array}\right)$ is increasing. Note that since $\xi_{j}$ preserves $C_{i, j}$, the morphism $\left(\begin{array}{cc}1 & -\xi \\ 0 & 1\end{array}\right)\left(\begin{array}{cc}c_{i, j} & 0 \\ 0 & c_{i, j}\end{array}\right)$ has image a based subcomplex satisfying the image order condition, namely $C_{i, j} \oplus S C_{i, j}$.

Finally all these chain maps have underlying basis functions the product of basis functions on the summands. Since the original simplex has commutative basis-function diagrams, so do the sums.

Cancellation conditions In the direct sum the contractions cancel complements because they do in the summands. In the cone $C_{j} \oplus_{1} S C_{j}$ projection of the contraction into the complement of a subcomplex of the form $\left(C_{i, j} \oplus S C_{i, j}\right)$ has a single nonzero entry, namely the identity from $\left(C_{j} \perp C_{i, j}\right)$ to $\left(S C_{j} \perp S C_{i, j}\right)$. The chain maps in (3) have the same image subcomplexes as the $i \rightarrow j$ structure maps in the cone, so the same cancellations work for them. This shows the $f_{i, j}$ define a $K_{1}$ morphism.

Finally (4) follows from the form of the contraction in the cone since it clearly cancels the entire complex. This concludes the proof of the lemma.

As explained above this implies

3.5.2 Corollary. Triangulation of the canonical morphisms of 3.5.1 gives a canonical homotopy of $i d \oplus S$ to 0 .

3.6 Comparison with Volodin $K$-theory. Volodin [Volodin 1] developed a version of $K$-theory using triangular matrices. This was shown to be the same as Quillen $K$-theory by [Vasserstein] and [Wagoner], see also the short (or at least highly compressed) proof by [Suslin]. Here we show there is a map from Volodin's space to $K_{1}(R)$ so that the induced map on loop spaces is a homotopy equivalence. This is the principal ingredient of the coefficient part of the controlled assembly isomorphism theorem 2.2.1, where a spectrum constructed from $K_{1}(R)$ is identified as the standard $K$-theory spectrum. The spectrum-level 
statement follows from the result here, the relatively formal facts that the map is a map of spectra, and isomorphism of higher homotopy implies isomorphism of lower homotopy by a suspension trick.

In this section we define Volodin's space and the map, and outline the proof.

3.6.1 Definition of $V_{k}(R)$. We use the description given by Suslin [S]. For each $k \geq 0$ define a simplicial set $V_{k}(R)$ whose $n$-simplices are sequences $g_{i}, 0 \leq i \leq n$,

(1) each $g_{i}$ is an isomorphism $R^{k} \rightarrow R^{k}$

(2) there exists a partial order on the basis so that each $g_{i} g_{j}^{-1}$ is 1 -triangular with respect to this order.

Recall that 1-triangular means triangular with diagonal entries 1 . The face map $\partial_{i}$ is defined by omitting the $i$ term in the sequence.

Next fix a standard countably generated free module $R^{\infty}$ and consider $R^{k}$ as the first $k$ coordinates. $R^{k}$ is then a based submodule of $R^{k+\ell}$, and isomorphisms of $R^{k}$ can be canonically extended to isomorphisms of $R^{k+\ell}$ by the identity in the other coordinates. This gives inclusions $\cdots \subset V_{k}(R) \subset V_{k+1}(R) \subset \ldots$ The Volodin space is the limit of this sequence:

$$
V(R)=\lim _{n \rightarrow \infty} V_{k}(R)
$$

3.6.2 The map $V(R) \rightarrow K_{1}^{V}(R)$. Suppose $\left(g_{*}\right)$ is an $n$-simplex of $V_{k}(R)$. Define an $n$-simplex of $K_{1}(R)$ by:

(1) chain complexes with modules $R^{k}$ in degrees 1 and 0 and trivial otherwise, with boundary $g_{i}: R^{k} \rightarrow R^{k}$ and contraction $g_{i}^{-1}$; and

(2) chain maps

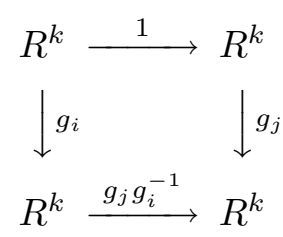

Note the cancellation and image agreement conditions are automatically satisfied because the chain maps are all isomorphisms. This construction defines a simplicial inclusion. Further it clearly commutes with the stabilizations $V_{k} \subset V_{k+1}$, so induces a map on the direct limit. The main result is: 
3.6.3 Theorem. The map $V(R) \rightarrow K_{1}(R)$ induces a homotopy equivalence of loop spaces.

Probably the most efficient way to prove this is to follow Suslin [S] by constructing a space with special $\pi_{1}$, showing it is acyclic, and appealing to a characterization theorem. We give a (rather elaborate) direct proof because some parts of it are useful in other circumstances. In the remainder of this section we set up notation and describe steps in this proof. Proofs of individual steps are given in the next four sections.

The first step is "compressing into two degrees" in 3.7. Define $K_{1}^{V}(R)$ to be the subcomplex of $K_{1}(R)$ consisting of complexes zero except in degrees 0 and 1 , then the result is that $K_{1}(R)$ deformation retracts to this. The map from the Volodin space maps into this subspace.

The second step in the proof, "standardizing dimensions", is given in 3.8. Define $K_{(\leq k)}^{V}(R)$ to be the subcomplex of $K_{1}^{V}(R)$ consisting of simplices whose chain modules have dimension $\leq k$. Similarly $K_{(=k)}^{V}(R)$ is the subcomplex of chain modules with dimension exactly $k$. We want to show these two subcomplexes are essentially the same. For convenience we work in the loop space. The result is that the loop space $\Omega K_{(\leq k)}^{V}(R)$ deformation retracts to $\Omega K_{(=k)}^{V}(R)$, and the retraction homotopy commutes with loops on stabilization:

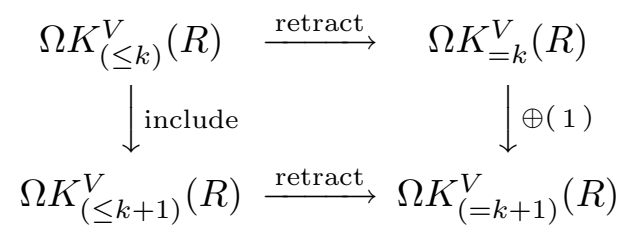

homotopy commutes. The subspaces $K_{(\leq k)}^{V}(R)$ form a direct system under inclusion, and $K_{1}^{V}(R)$ is the union. The result shows it is also the limit of $K_{(=k)}^{V}(R)$ under stabilization. The map $V_{k}(R) \rightarrow K^{V}(R)$ has image in $K_{(=k)}^{V}(R)$ and these maps give a morphism of direct systems. Therefore to prove Theorem 3.6.3 it is sufficient to prove the unstable analog: the map $V_{k}(R) \rightarrow K_{(=k)}^{V}(R)$ induces a homotopy equivalence of loop spaces.

The third step is "rolling out" in 3.9, and again takes place in loop spaces. 1-simplices of $K_{(=k)}^{V}(R)$ are triangular chain maps of complexes of length 2, so correspond to changing boundary homomorphisms by composition on both the right and left by triangular isomorphisms. The Volodin space is constructed 
using cosets, so uses composition only on one side. To fix this we consider a loop $K_{(=k)}^{V}(R)$, which roughly corresponds to a sequence of 2-sided compositions giving the identity. But then we can slide things past the identity to move them all to one side. This deforms the loop space of $K_{(=k)}^{V}(R)$ to the loop space of $V_{k}^{ \pm}(R)$, the subspace in which the degree-1 part of chain maps in 1-simplices are identities.

The final step, "fixing signs" in 3.10, shows the loop space of $V_{k}^{ \pm}(R)$ deformation retracts to the (image of) loop space of the Volodin space $V_{k}(R)$. The difference between the two spaces is that $V_{k}(R)$ is defined using +1-triangular morphisms, while \pm 1 -triangular ones are allowed in $V_{k}^{ \pm}(R)$. The negative signs are a consequence of using chain complexes, and cannot be avoided. However in the loop space they can be removed essentially by taking absolute values on the diagonal.

Together these steps prove the comparison Theorem 3.6.3.

3.7 Compressing into two degrees. Define $K_{1}^{V}(R)$ to be the subcomplex of $K_{1}(R)$ consisting of complexes that are trivial in all degrees except 0 and 1 . In the next section we analyse this subcomplex; here the objective is to show

3.7.1 Theorem. There is a canonical deformation retraction of $K_{1}(R)$ to $K_{1}^{V}(R)$.

The proof uses "degree-wise localization" of the cancellation of inverses. "Localization" will refer to the use of the steps of 3.4 to cancel some part of a complex but leave other parts alone. In later sections we use reference maps to a space to determine which parts to cancel. Here we use degrees, roughly speaking cancelling in positive degrees and leaving degree 0 alone.

The proof is a modification of the infinite swindle that contracts $K_{1}(R)$, but goes through infinite complexes that are nontrivial in negative degrees. Recall that the suspension $S^{j} C$ of a complex is defined (3.1.3) by shifting the grading by $j$. Consider the map that takes $C$ to infinite sum $\oplus_{j \geq 0} S^{-j} C$. We write this sum as $\cdots \oplus S^{-j} C \oplus \cdots \oplus S^{-1} C \oplus C$ for later notational convenience. If we associate this in pairs as $\cdots \oplus\left(S^{-2 i-1} C \oplus S^{-2 i}\right) \oplus \cdots$ then applying the cancellation homotopy to the pairs gives a homotopy of the map to the constant 0 map. If we associate as $\cdots \oplus\left(S^{-2 i} C \oplus S^{-2 i+1}\right) \oplus \cdots \oplus C$ and apply the cancellation homotopy then all the nontrivial suspensions vanish and we get a homotopy to the identity map $C \mapsto C$. Putting these together gives a nullhomotopy of the inclusion into a space 
of infinite complexes. Note however that since we used negative suspensions the nonnegative-degree parts of the intermediate complexes are finite. The plan is roughly to get back into $K_{1}(R)$ by discarding everything in negative degrees. We cannot literally do this because truncation does not "commute" with cancellation of inverses. However we can limit the problems to degrees 0 and 1 . The outcome will be a homotopy that agrees with the swindle in degrees 2 and above, and stays in finitely generated nonnegative complexes. The end of the homotopy is no longer completely trivial, but is concentrated in degrees 0 and 1 . Finally we arrange that if a complex is already concentrated in degrees 0 and 1 then the homotopy leaves it unchanged. This gives the deformation retraction promised in the theorem.

To fill out this outline we must describe how the cancellations in the infinite swindle are "localized" to positive degrees. To do this we write out low degree groups in each step, and display chain maps, contractions, etc. explicitly as matrices. We leave to the reader the verifications that these actually are chain maps and the contractions cancel inverses. As in 3.5 the process is canonical, so it is sufficient to describe the effect on a single simplex $(C, \xi)$.

3.7.2 Stabilization. The first step is to stabilize by including $C$ into the sum with mapping cones of identity morphisms. The complexes involved are even negative suspensions with negative degrees omitted: $S_{0}^{-2 i} C$ denotes the complex with degree- $j$ group $C^{j-2 i}$ if $j-2 i \geq 0$, and 0 otherwise. The mapping cone is the sum of this with its suspension, which is the $(-2 i+1)$ suspension of $C$ truncated in degree 1. In the following diagram the group in a single degree is obtained by summing a horizontal row, and arrows indicate nontrivial components of the boundary homomorphisms:
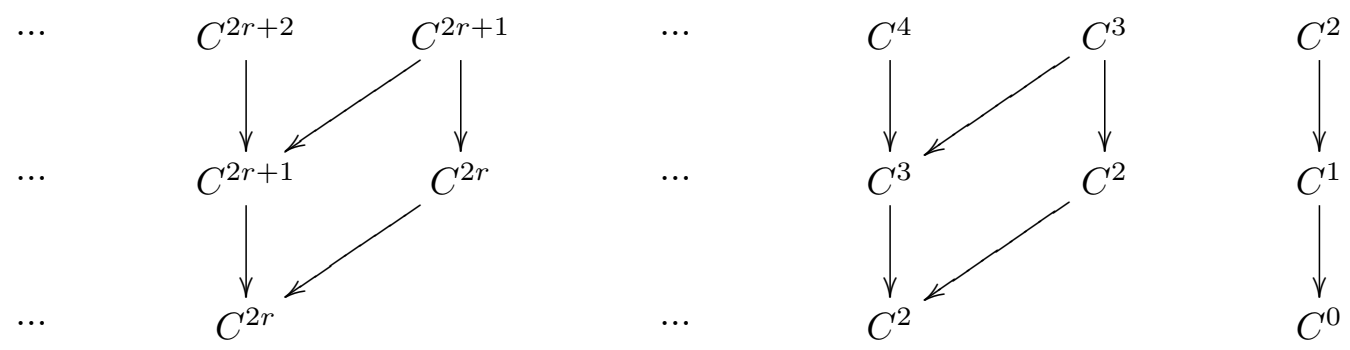
The boundary in this complex is $\left(\begin{array}{lll}\ddots & & \\ & c & 1 \\ & 0 & -c \\ & & c\end{array}\right)$ in degrees $\geq 2$, and $\left(\begin{array}{lll}\ddots & & \\ & c & 1 \\ & & c\end{array}\right)$ on degree 1. The contraction is $\left(\begin{array}{lll}\ddots & & \\ & 0 & 0 \\ & 1 & 0 \\ & & \xi\end{array}\right)$ in degrees $\geq 1$ and $\left(\begin{array}{lll}\ddots & \\ & 0 \\ & 1 & \\ & & \end{array}\right)$ in degree 0 .

3.7.3 Reparameterization. The next step is to use, as much as possible, the isomorphism of 3.5.1 from this to a complex with the same groups and a direct sum boundary structure. The isomorphism in degrees $\geq 1$ is $\left(\begin{array}{lll}\ddots & \\ & 1 & -\xi \\ 0 & 1\end{array}\right)$, and we use the identity in degree 0. Specifying the chain maps determines the boundary structure in the range. This is $\pm c$ on the diagonal (as desired) in degrees $\geq 2$, and $\left(\begin{array}{ccc}\ddots & & \\ & \xi c & \\ & c & \\ & & c\end{array}\right)$ in degree 1 . The contraction is $\pm \xi$ on the diagonal in degrees $\geq 2$. The contraction is $\left(\begin{array}{cccc}\ddots & & & \\ & c \xi & 0 & \\ & 0 & c \xi & \\ & & & c \xi\end{array}\right)$ in degree 1 and $\left(\begin{array}{ccc}\ddots & \\ & \xi c \xi \\ & \xi c & \\ & & \xi\end{array}\right)$ in degree 0.

The second morphism goes from a complex with the same groups to this. It is defined like the first morphism but with the association of terms shifted over by one: $\left(\begin{array}{cccc}\ddots & & & \\ & 1 & -\xi & \\ 0 & 1 & & \\ & & 1 & -\xi\end{array}\right)$ in positive degrees, and the identity in degree 0 . We work out that the boundary homomorphism in the complex is a mapping cone in degrees $\geq 2$ : $\left(\begin{array}{cccc}\ddots & & & \\ & -c & 0 & \\ & 1 & c & \\ & & -c & 0\end{array}\right)$, and in degree 1 is $\left(\begin{array}{cccc}\ddots & & & \\ & & -\xi c \xi & \\ & c & \xi c & \\ & & & -\xi c \xi\end{array}\right)$. Diagrammatically this is 


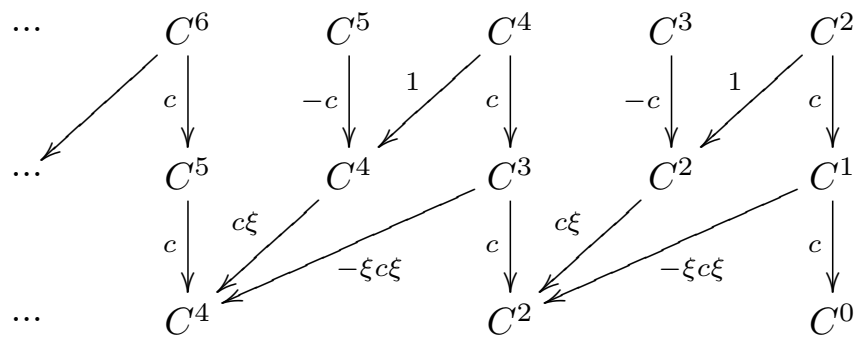

The contraction is the mapping cone contraction in degrees $\geq 2$, is $\left(\begin{array}{cccc}\ddots & & & \\ & 0 & 0 & \\ & c \xi & 0 & \\ & & 0 & 0 \\ & c \xi & 0\end{array}\right)$ in degree 1 , and $\left(\begin{array}{llll}\ddots & & & \\ & -\xi c \xi & 0 & \xi \\ & & -\xi c \xi 0 \xi\end{array}\right)$ in degree 0.

3.7.4 Cancellation. The final step is to note that the contractions allow us to omit most of this. The residual subcomplex consists of the bottom two groups in even-numbered summands, and inclusion into the whole thing is a $K_{1}$ morphism.

The residual complex is concentrated in degrees 0 and 1. Explicitly it is: in degree $0, \oplus_{* \text { even }} C^{*}$, in degree $1, \oplus_{* \text { odd }} C^{*}$, the boundary is $c-\xi c \xi$, and the contraction is $\xi c \xi-c \xi c$. We recognize this as a variant on the Whitehead formula for the torsion of a contractible complex. The morphisms described above give a homotopy from the identity of $K_{1}(R)$ to the map that takes $C$ to the complex given by this formula, so into the subcomplex $K_{1}^{V}(R)$ as required. Finally note that if $C$ is already concentrated in degrees 0 and 1 then it is left unchanged by all of this.

This completes the proof of the two-degree theorem.

3.8 Standardizing dimensions. In $K_{1}$ simplices dimensions are allowed to increase provided the contraction cancels the new part. Here we deform simplices to ones where all dimensions are the same, roughly by using the cancellation information to fill out the small ones. Define (as in 3.6) $K_{(\leq k)}^{V}(R)$ to be the subcomplex of $K_{1}^{V}(R)$ consisting of simplices of complexes with dimension $\leq k$ in each degree. $K_{(=k)}^{V}(R)$ is the subcomplex of complexes with dimension exactly $k$ in degrees 0 and 1 . 
3.8.1 Proposition. The loop space $\Omega K_{(\leq k)}^{V}(R)$ deformation retracts to $\Omega K_{(=k)}^{V}(R)$ so that the retraction homotopy commutes with inclusion and suspension:

$$
\begin{array}{rr}
\Omega K_{(\leq k)}^{V}(R) & \stackrel{\text { retract }}{\longrightarrow} \Omega K_{=k}^{V}(R) \\
\downarrow \downarrow \text { include } & \\
\Omega K_{(\leq k+1)}^{V}(R) \stackrel{\text { retract }}{\longrightarrow} \Omega K_{(=k+1)}^{V}(R)
\end{array}
$$

3.8.2 Details on $K_{1}^{V}(R)$. The definition of $K_{1}$ simplices simplifies quite a bit for complexes concentrated in two dimensions. An $n$-simplex consists of:

(1) (short contractible complexes) for $0 \leq i \leq n$ an isomorphism $c_{i}: C_{i}^{1} \rightarrow$ $C_{i}^{0}$

(2) (chain maps) for $i<j$, homomorphisms $c_{i, j}^{*}: C_{i}^{*} \rightarrow C_{j}^{*}$, where $*=0,1$, such that the diagram commutes:

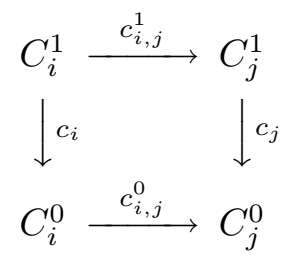

(3) (partial orders) there exist partial orders on the bases of $C_{i}$ in each degree so that $c_{i, j}$ is \pm 1 triangular, and the image of the basis function of $c_{i, j}$ is greater than its complement;

(4) (commutative basis functions) if $i \leq j \leq k$ then $c_{j, k} c_{i, j}$ has the same basis function as $c_{i, k}$; and

(5) (cancellation condition) the restriction of $c_{j}$ to the based complements of $c_{i, j}^{*}$ is \pm 1 triangular.

This relates to definition 3.4.1 as follows: a complex of length 2 is contractible if and only if the boundary is an isomorphism, and in this case there is a unique contraction (the inverse). Condition (1) thus encodes contractible complexes. Condition (2) is equivalent to the $c_{i, j}^{*}$ defining chain maps. (3) encodes the triangularity hypothesis and the image order condition. (4) is the same as in 3.4.1. (5) encodes the cancellation property of the contraction.

The next result is a version of the "standard coordinates" for cancellations given in 3.3.2. 
3.8.3 Lemma. Suppose $f: B \rightarrow C$ is a $K_{1}^{V}$ morphism (1-simplex) and $\phi: R^{\ell} \rightarrow$ $C^{1}$ gives a based isomorphism to the complement of the image $f\left(B^{1}\right)$. Then the isomorphism $\hat{f}$ given by $\left[f^{1}, \phi\right]$ in degree 1 gives a $K_{1}^{V}$ commutative diagram (2-simplex)

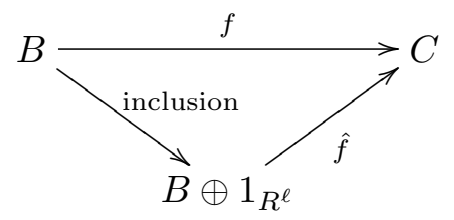

More precisely any partial orders on $B, C$ making $f$ a 1 -simplex determines one on the stabilization making the diagram a 2 -simplex. In degree $0 \hat{f}$ is given by $\left[f^{0}, c \phi\right]$.

To see $K_{1}^{V}$ structure we decompose $C$ as the image of $f$ plus a cancellation part: $C^{1}=\bar{C}^{1} \oplus D$ and $C^{0}=\bar{C}^{0} \oplus \bar{D}$. The chain map $\hat{f}$ in these coordinates is:

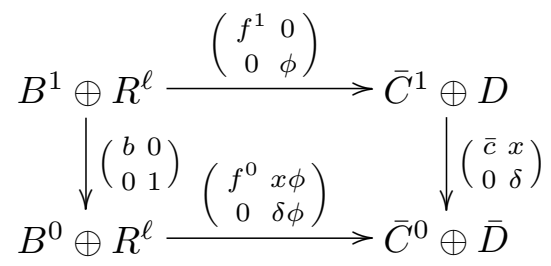

Since $\phi$ is a based isomorphism it is 1-triangular with respect to any partial order. Use the partial order on $B^{1} \oplus R^{\ell}$ that makes the basis map of $\hat{f}^{1}$ orderpreserving. This extends the partial order on $B^{1}$ and makes $\hat{f}^{1} \pm 1$ triangular because $f^{1}$ is. Further $\hat{f}^{0}$ is \pm 1 triangular because the basis of $R^{\ell}$ preceeds the basis of $B^{0}$ (by 3.8.2(3)) and $f^{0}, \delta$ and $\phi$ are all \pm 1 triangular. This means $\hat{f}$ is a $K_{1}^{V}$ morphism with respect to the given partial orders. Finally the diagram in the lemma commutes, so by 3.2.3 in each degree the underlying basis functions commute. This means the diagram is a 2 -simplex in $K_{1}^{V}$.

3.8.4 Loop spaces. The model we use for the loop space uses triangulations of $\Delta^{n} \times I$ such that the projection to $\Delta^{n}$ is simplicial. There is a standard minimal such triangulation described in 3.4.3. All such triangulations have qualitative structure similar to the standard one: there is a linear ordering of the $(n+1)$ simplices so adjacent ones share a face, and the projection of each one to $\Delta^{n}$ collapses a single edge. 
If $K$ is a simplicial space define the loop space $\Omega(K, *)$ to be the simplicial space with $n$-simplices simplicial maps of triangulations of $\Delta^{n} \times I$ as above, with $\Delta \times\{0,1\}$ mapping to the basepoint. Boundaries of simplices are defined by restricting maps to faces $\left(\partial_{i} \Delta^{n}\right) \times[0,1]$.

3.8.5 Proof of Proposition 3.8.1. Take the identity map 1: $R^{k} \rightarrow R^{k}$ as the basepoint of both $K_{\leq k}^{V}$ and $K_{=k}^{V}$. We construct a retraction $S: \Omega K_{\leq k}^{V} \rightarrow K_{=k}^{V}$ and a morphism $s$ : id $\rightarrow S$. The deformation of the lemma is obtained by subdividing the morphism. The retraction and morphism are constructed by induction on skeleta. More precisely we construct them on the vertices, then extend to the 1-simplices. Higher dimensional simplices are defined to be collections of 1simplices satisfying some coherence conditions, so no further constructions are required, but coherence must be checked.

A vertex of $\Omega K_{(\leq k)}^{V}$ is a simplicial map of a triangulation of $I$ into $K_{(\leq k)}^{V}$, with the ends going to $1_{R^{k}}$. Such a map is a sequence of objects $C_{0}, \ldots C_{n}$ with morphisms between adjacent ones: either $C_{i} \rightarrow C_{i+1}$ or $C_{i} \leftarrow C_{i+1}$. Define objects $\hat{C}_{i}$ by stabilizing the $C_{i}$ to have dimension $k: \hat{C}_{i}=C_{i} \oplus\left(1: R^{\ell} \rightarrow R^{\ell}\right)$, where $\ell+\operatorname{dim}\left(C_{i}^{1}\right)=k$. We want to fill in the dotted arrows in the following diagram so the squares are $K_{1}^{V}$ morphisms (including coherent partial orders):

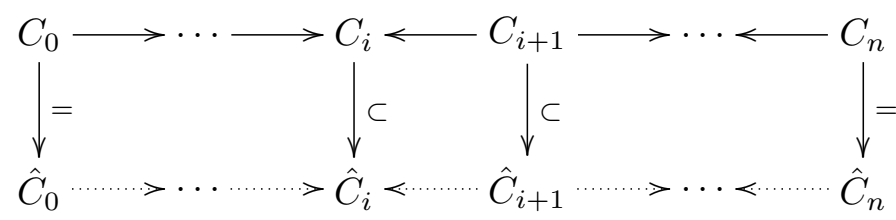

Consider a square in which the morphism goes from $C_{i}$ to $C_{i+1}$, and choose appropriate partial orders. Partial order the bases in $\hat{C}_{i+1}$ by putting the new elements first, then the inclusion is a morphism and the composition (diagonal in the following) is also a morphism.

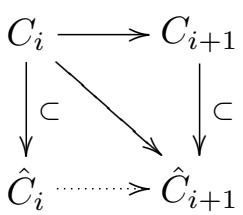

According to Lemma 3.8.3 a based isomorphism from the stabilization factor $R^{\ell}$ in $\hat{C}_{i}^{1}$ to the complement of the image in $\hat{C}_{i+1}^{1}$ determines a way to complete the 
lower triangle. There is such an isomorphism for dimension reasons; choose one randomly and use that.

Squares in which the morphism goes from $C_{i+1}$ to $C_{i}$ are filled in with the mirror image of this argument.

Note the ends of the sequence already have dimension $k$ so do not change. This means the lower row in the diagram gives a sequence that begins and ends with identities, so defines a vertex in $\Omega K_{(=k)}^{V}$. The rest of the diagram defines a morphism from the original vertex in $\Omega K_{(\leq k)}^{V}$ to this. This defines the retraction $S$ and morphism $s$ on vertices.

Now consider a 1-simplex in $\Omega K_{(\leq k)}^{V}$. This is a map of a triangulation of $\Delta^{1} \times I$ into $K_{(\leq k)}^{V}$, so consists of two vertices as above and a sequence of 2simplices filling in between them. Denote the vertices by $C_{*}$ and $D_{*}$, then a typical segment looks like

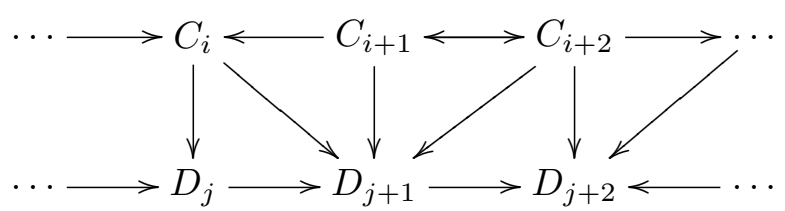

The retraction and morphism are already defined on vertices, so have sequences $\hat{C}_{*}, \hat{D}_{*}$ and morphisms from the originals to these. To extend $S$ to the 1 -simplices we need to fill in 2-simplices joining $\hat{C}_{*}$ and $\hat{D}_{*}$. We do this by working along the loop coordinate. Note since loops begin at the identity the identity $\hat{C}_{0}=\hat{D}_{0}$ fills in the beginning of the diagram. Suppose by induction that we have extended it up to a 2 -simplex with one vertex on $C_{i}$, so have everything but the dotted arrow in the following:

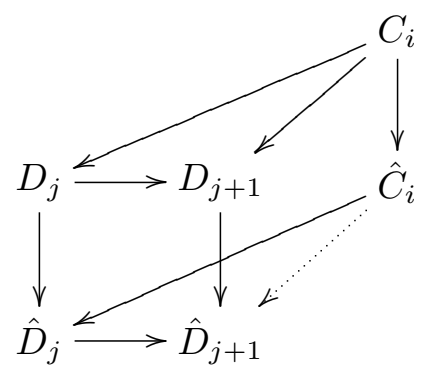

Again 3.8.3 can be used to fill this in. Compose $C_{i} \rightarrow D_{j+1} \rightarrow \hat{D}_{j+1}$ to obtain the setting of 3.8.3. Now however we use a particular basis-preserving isomorphism from the stabilization factor in $\hat{C}_{i}$ to the complement in degree 1 in $\hat{D}_{j+1}$. 
Namely, the composition $\hat{C}_{i} \rightarrow \hat{D}_{j+1} \rightarrow \hat{D}_{j+1}$ is a \pm 1 triangular isomorphism, and the underlying basis function preserves images, etc. so gives a bijection. Applying 3.8.3 using this gives a dotted arrow so that the right square commutes and basis functions commute. This fills in this triangle, and we move on to the next. Triangles with two vertices on the $C_{*}$ are handled similarly.

Eventually this process reaches the end of $\Delta^{1} \times I$. There nothing happens since the dimension is already $k$, so the diagram ends with identities. The $\hat{C}$, $\hat{D}$ part of the diagram gives a 1-simplex in $\Omega K_{(=k)}^{V}$ and we define this to be the image of $S$. The morphisms going from the original to the new part give a morphism from the original 1-simplex to the new one, and this is defined to be $s$.

Finally consider an $n$-simplex of $\Omega K_{(\leq k)}^{V}$. The construction on 0 - and 1simplices gives all the objects and maps required to define $S$ and $s$ on this simplex, but we need to check \pm 1 triangularity with respect to coherent partial orders, and commutativity of underlying basis functions. The ability to choose coherent partial orders follows from the refinement noted after the statement of 3.8.3 that any suitable partial orders on the input extends to ones on the output. To see the underlying basis functions commute it is sufficient to check 2 -simplices. There it is easily seen to hold by the choice made in construction of 1-simplices.

This $S$ maps to $\Omega K_{(=k)}^{V}$. It is a retraction (identity on $\Omega K_{(=k)}^{V}$ ) because nothing happens when dimensions are already $k$. This completes the proof of the dimension-standardizing proposition.

3.9 Rolling out. Recall ( $\S 3.6)$ that $V_{k}^{ \pm}$is defined like $V$ but with \pm 1 triangular automorphisms of $R^{k}$. It maps to $K_{(=k)}^{V}$, essentially to the subspace of short chain complexes in which the degree-1 part of chain maps are base-preserving isomorphisms. The basic difference between $K_{(=k)}^{V}$ and $V_{k}^{ \pm}$is that in the former the morphisms change objects by composition on both sides with triangular matrices, whereas in the latter compositions are only on one side.

3.9.1 Proposition. The inclusion of loop spaces $\Omega V_{k}^{ \pm} \rightarrow \Omega K_{(=k)}^{V}$ is a homotopy equivalence of realizations.

The plan of the proof is to define a filtration $\Omega V_{k}^{ \pm}=X_{0} \subset X_{1} \subset \ldots$ with $\cup_{i} X=\Omega K_{(=k)}^{V}$. We then construct a homotopy from the identity of $K_{(=k)}^{V}$ to a map $S$ so that $S$ decreases filtration degrees (i.e. $S\left(X_{i}\right) \subset X_{i-1}$ ) and the 
homotopy increases degrees in a controlled manner (the image of the homotopy on $X_{i}$ is in $X_{2 i}$ ). The proposition follows routinely from this.

3.9.2 The filtration. An $n$-simplex of $\Omega K_{(=k)}^{V}$ is a special triangulation of $\Delta^{n} \times$ $I$ and a simplicial map of this to $K_{(=k)}^{V}$ that takes the ends to the basepoint id: $R^{k} \rightarrow R^{k}$. For instance a vertex is a triangulation of $I$ and a map to $K_{(=k)}^{V}$, so a linear sequence of short chain complexes and maps. Since the maps are all invertible, for notational convenience we can take them all to go in the direction of the loop coordinate. Explicitly we have $k$-dimensional based modules and isomorphisms:

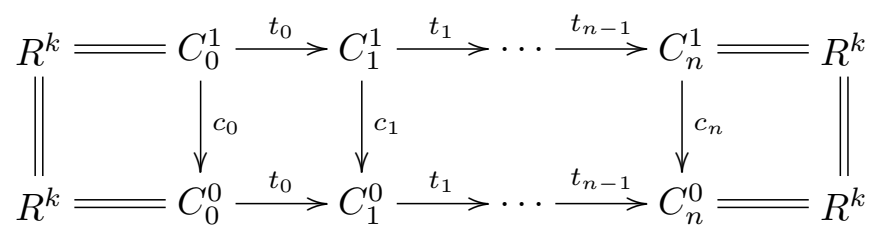

The $n+1$-simplices in these triangulations of $\Delta^{n} \times I$ have a linear order so that adjacent ones share a face, the first has $\Delta^{n} \times\{0\}$ as a face, and the last has $\Delta^{n} \times\{0\}$ as a face. We define a map from this triangulation into $K_{(=k)}^{V}$ to be in the subspace $X_{r}$ if all but the last $r$ of these $n+1$-simplices map into $V_{k}^{ \pm}$.

3.9.3 The homotopy. This construction is canonical so it is sufficient to describe the effect on a simplex. In fact we only describe it on vertices. It is straightforward to extend this description to 1-simplices, but the diagrams are too complicated to be useful and are best drawn for oneself. The general case follows from 1-simplices and easily-seen coherence of partial orders.

Begin with a simplex, which is a diagram as in 3.9.2. The first step in the homotopy doubles the length of the diagram by alternating the given chain maps with identities. The degree- 1 terms of the chain maps are shifted one place to the right by starting with an identity. There is a "morphism" (a 1-simplex) going from this new vertex to the old one, giving the homotopy. In the diagram the front row is the original vertex, the back row is the new one, and the morphisms joining them give the homotopy. Identity maps are denoted by long equality 
signs.

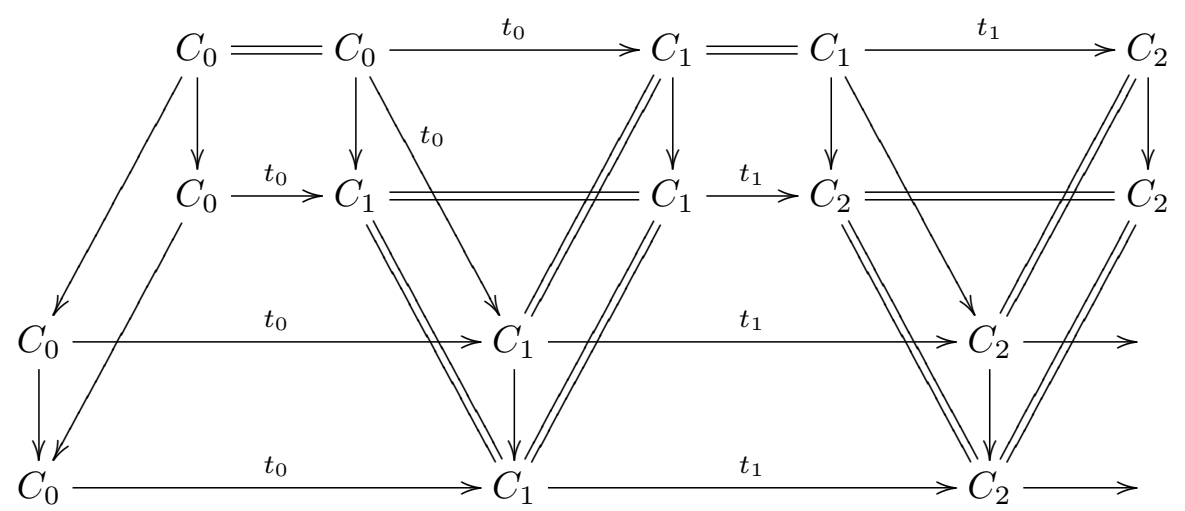

Note that the stretching takes vertices in the filtration $X_{r}$ to $X_{2 r-1}$.

The next step is to apply the inverse of this move on the segment omitting the first and last squares. The result is a sequence with length one more than the starting sequence and with the degree- 1 terms of the chain maps shifted right one position. This sequence begins with a chain map that is the identity in degree 1 , and ends with one that is the identity in degree 0 .

Repeat this sequence of moves. This takes us to a sequence of length greater by 2 , with the last two squares the identity in degree 0 , explicitly the sequence ends with

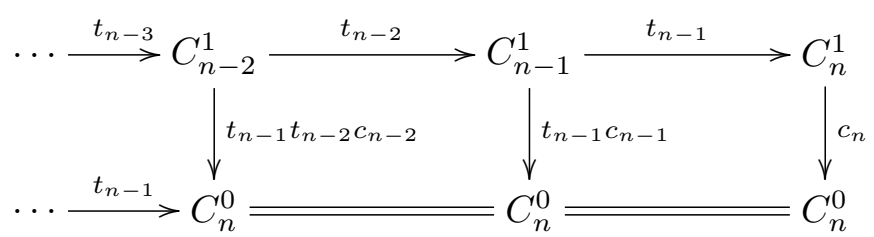

This vertex is in the same filtration degree as the original one because the filtration is defined using the end of the degree- 1 part of the chain maps, and this has not changed.

The final step in the homotopy shortens this diagram by 1 by moving the end degree- 1 term down to degree 0 . In the diagram the beginning sequence is in the 
back, the end in the front.

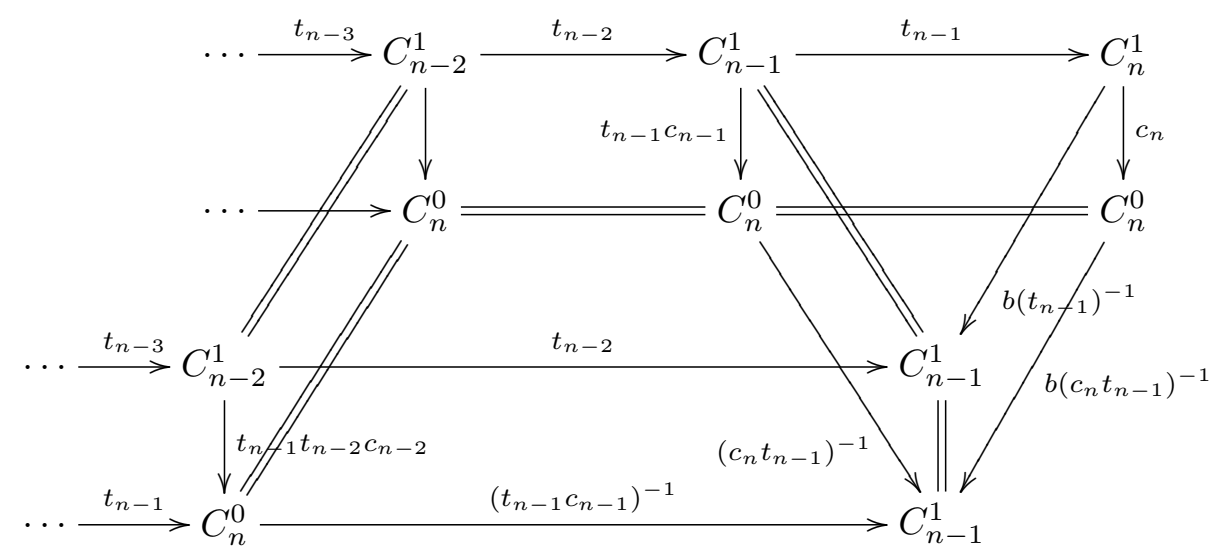

On the right-hand slanted arrows " $b(*)$ " denotes the underlying basis function of the triangular map $*$. Note $t_{*}$ is triangular because it is part of a chain map in a 1-simplex, and $c_{n}$ is a based isomorphism (in fact identified with id: $R^{k} \rightarrow$ $\left.R^{k}\right)$. The diagrams all commute except for the triangles on the right which are off by the triangular parts of the inverses of $t_{n-1}$ and $c_{n} t_{n-1}$ respectively. Recall, however, that 2-simplices in $K_{(=k)}^{V}$ are not required to commute. The requirements are that triangularity conditions should all hold with respect to fixed choices of partial order, and the underlying basis functions should commute. These conditions clearly do hold. The diagram therefore defines a 1-simplex. The new vertex at the end of the simplex has one fewer non-basis-preserving map in degree 1 , so is in lower filtration degree than the original. This accomplishes the objective of the construction.

As noted above it is straightforward to extend this to 1-simplices, but the diagrams have twice as many terms so we will not attempt them here. Higher simplices are defined by collections of 1-simplices satisfying coherence conditions on partial orders and basis functions. Since the construction is canonical this follows formally.

3.10 Fixing signs. This section gives the last step in the proof of Theorem 3.6.3:

3.10.1 Proposition. The loop space $\Omega V_{k}^{ \pm}$deformation retracts to $\Omega V_{k}$.

Recall that an $n$-simplex in $V_{k}^{ \pm}$is a sequence of automorphisms of $R^{k}$ (matrices) $\left(g_{0}, \ldots, g_{n}\right)$ so that all products $g_{j} g_{i}$ with $j>i$ are all \pm 1 triangular with 
respect to some partial order of the basis of $R^{k}$. The next lemma asserts that we can change the signs on the diagonal arbitrarily.

3.10.2 Lemma. Suppose $\left(g_{i}\right)$ is an n-simplex of $V_{k}^{ \pm}$and $\left(\epsilon_{0}, \ldots, \epsilon_{n}\right)$ are \pm 1 diagonal matrices. Then $\left(\epsilon_{i} g_{i}\right)$ is also an $n$-simplex and $\left(g_{i}\right) \rightarrow\left(\epsilon_{i} g_{i}\right)$ is a morphism of simplices.

Here "morphism" of simplices should be interpreted using the images in $K^{V}$. More directly it means it gives a homotopy of the simplex defined on the standard triangulation of $\Delta^{n} \times I$, with $n+1$-simplices of the form $\left(g_{0}, \ldots, g_{i-1}, \epsilon_{i} g_{i}, \ldots, \epsilon_{n} g_{n}\right)$. To check these are simplices use a partial order that makes $\left(g_{i}\right)$ a simplex. The products in one of these $n+1$-simplices are of the form $g_{j} g_{i}^{-1}, \epsilon_{j} g_{j} g_{i}^{-1}$, and $\epsilon_{j} g_{j} g_{i}^{-1} \epsilon_{i}^{-1}$. These are all \pm 1 triangular because the $g_{j} g_{i}^{-1}$ are and the $\epsilon_{j}$ are \pm 1 triangular with respect to any partial order.

3.10.3 Proof of the Proposition. We begin by defining the map $S$ and homotopy $s$ on vertices. A vertex of $\Omega V_{k}^{ \pm}$is a sequence $\left(g_{0}, \ldots g_{r}\right)$ of $k \times k$ matrices with $g_{0}=\mathrm{id}=g_{r}$ and for each $i$ there is a partial order in which $g_{i} g_{i-1}^{-1}$ is \pm 1 triangular. Inductively define $\epsilon_{i}$ by $\epsilon_{0}=\mathrm{id}$, and $\epsilon_{i}=\operatorname{diag}\left(g_{i} g_{i-1}^{-1} \epsilon_{i-1}^{-1}\right)$. The matrix on the right is \pm 1 triangular with respect to some partial order, so the diagonal part is a well-defined \pm 1 diagonal matrix (Lemma 3.2.4). Define $S$ on this path by

$$
S\left(g_{*}\right)= \begin{cases}\left(\epsilon_{0} g_{0}, \ldots, \epsilon_{r} g_{r}\right) & \text { if } \epsilon_{n}=\mathrm{id} \\ \left(\epsilon_{0} g_{0}, \ldots, \epsilon_{r} g_{r}, \text { id }\right) & \text { if not }\end{cases}
$$

According to the lemma this is a path in $V_{k}^{ \pm}$, and there is a morphism (standard homotopy) from the original path to the new one. (The second case requires sticking on a trivial 2-simplex at the end of the path).

The choice of the $\epsilon_{i}$ implies the image path is actually in $V_{k}$, and is unchanged if the original path were already in this subspace.

Lemma 3.10.2 implies that this map and homotopy extend to higher simplices of $\Omega V_{k}^{ \pm}$. This is because the higher simplices are determined by their vertices, on which the map is defined, and triangularity of products with respect to a single partial order. The lemma asserts that this triangularity holds.

The extension to all simplices of $\Omega V_{k}^{ \pm}$gives a map into $\Omega V_{k}$ that is the identity on $\Omega V_{k}$, and a homotopy of this to the identity in $\Omega V_{k}^{ \pm}$. This is the conclusion of Proposition 3.10.1. 


\section{Controlled $K$-theory}

In this section we give the definition and naturality properties that do not require spacial localization. General definitions for controlled algebra are in $\S 4.1$, and differ in many details from versions used earlier. These differences do not change the final outcomes but make some arguments easier. The controlled version of triangularity is described in 4.2 . The controlled $K_{1}$ space is defined in $\S 4.3$, essentially by inserting many $\epsilon$ s into the uncontrolled definition. Naturality with respect to restriction and uniformly continuous functions are immediate consequences of the definition. Dependence on the map $E \rightarrow X$ is also analysed in this section. The inverse limit as $\epsilon \rightarrow 0$ is introduced in Section 4.4.

4.1 Controlled algebra. The definitions we use for controlled algebra are different from other versions, particularly in the systematic use of paths. An earlier version of path-based definitions is in [Quinn 6], see also [Anderson-Munkholm]. Metric notations are given in $\S 4.4 .1$, modules described in 4.1.2, and homomorphisms defined in $\S 4.1 .3$.

4.1.1 Metric notions. Suppose $X$ is a metric space with metric $d$. We say a path $\gamma:[0,1] \rightarrow X$ has radius $<\epsilon$ if $d(\gamma(s), \gamma(t))<\epsilon$ for all $s, t \in[0,1]$.

Now suppose $\epsilon>0$ and $Y \subset X$. Define the " $\epsilon$ (path) enlargement" $Y^{\epsilon}$ to be all points that can be reached from $Y$ by a path of radius $<\epsilon$. Previously we have used the notation $Y^{\epsilon}$ to denote the points with distance less than $\epsilon$ from $Y$, but the truth is that we don't care how close a point is if you can't get there by a small path. There is a standard way to redefine the metric using paths so distances encode path properties, and when this is done the two definitions coincide. We prefer not to do this because it complicates consideration of subspaces.

In the opposite direction define the " $\epsilon$ (path) reduction" to be points that cannot be reached from the complement of $Y$ by a path of radius $<\epsilon$. Note $Y^{-\epsilon}=X-(X-Y)^{\epsilon}$.

The ambient space $X$ is not displayed in this notation. It is usually clear from the context but occasionally, as in the lemma below, will require comment. A benefit of using path enlargements is that they are less dependent on the ambient space than distance enlargements, as the following "excision" lemma shows:

Lemma. Suppose $U$ is open in $X$. Then $(X-U)^{\epsilon} \cap U=(\bar{U}-U)^{\epsilon} \cap U$, where 
$\bar{U}$ denotes the closure in $X$, the path enlargement on the left is taken in $X$, the one on the right in $\bar{U}$.

Proof. Since $(\bar{U}, \bar{U}-U) \subset(X, X-U)$ the containment $(X-U)^{\epsilon} \cap U \supset(\bar{U}-U)^{\epsilon} \cap U$ is clear. To show the other direction suppose $x \in(X-U)^{\epsilon} \cap U$. This means there is a path $\gamma:[0,1] \rightarrow X$ of radius $<\epsilon$ starting at $x$ and ending in $X-U$. Let $t_{0}$ be the minimum of $\{t \mid \gamma(t) \notin U\}$. Since $U$ is open $\gamma\left(t_{0}\right) \notin U$, but it is in the closure. Thus $\gamma \mid\left[0, t_{0}\right]$ is a path of radius $<\epsilon$ in $\bar{U}$ starting at $x$ and ending in $\bar{U}-U$. This shows $x \in(\bar{U}-U)^{\epsilon}$, as required.

4.1.2 Metric frontiers. The metric frontier of a metric space is the complement of $X$ in its completion, and is denoted by $\operatorname{Fr}(X)$. Technically this is the set of limit points of Cauchy sequences in $X$ that do not converge in $X$ itself. Note that if $X$ is complete (e.g. compact) then the metric frontier is empty.

We use the shorthand notation $\operatorname{Fr}^{\epsilon} X$ for $X \cap(\operatorname{Fr}(X))^{\epsilon}$, where the enlargement $(*)^{\epsilon}$ is taken in the metric closure. $\operatorname{Fr}^{\epsilon} X$ can also be described as beginning points of non-convergent Cauchy paths $\gamma:[0,1) \rightarrow X$ of radius $<\epsilon+\delta$ for some positive $\delta$.

These definitions can differ from casual expectations in spaces that are not locally path-connected. For example if $X$ is the sequence of real numbers $\left\{\frac{1}{n}\right\}$ then the metric frontier of $X$ is $\{0\}$. The path enlargement of $\{0\}$ in $\left\{\frac{1}{n}\right\} \cup\{0\}$ is just $\{0\}$, so $\operatorname{Fr}^{\epsilon} X$ is empty. This is what we want, however, because geometric algebra is defined using paths.

\section{Lemma.}

(1) If $U \subset X$ is open then $\left(\operatorname{Fr}^{\epsilon} X \cup(\bar{U}-U)^{\epsilon}\right) \cap U=\operatorname{Fr}^{\epsilon} U$.

(2) if $f: X \rightarrow Y$ is a map of metric spaces such that $d\left(x, x^{\prime}\right)<\delta$ implies $d\left(f(x), f\left(x^{\prime}\right)\right)<\epsilon$, then $f\left(W^{\delta}\right) \subset(f(W))^{\epsilon}$.

(3) if $f: X \rightarrow Y$ is proper and uniformly continuous then it extends to a map of completions with $\bar{f}(\operatorname{Fr} X) \subset \operatorname{Fr} Y$.

Note that if the hypotheses of both (2) and (3) hold then $\left.f\left(\operatorname{Fr}^{\delta} X\right) \subset \operatorname{Fr}^{\epsilon} Y\right)$.

Some of the notation in statement (1) is a bit ambiguous. $\bar{U}$ indicates the closure in $X$. The effect of adding $\operatorname{Fr}^{\epsilon} X$ in is the Lemma of the previous section applied in the metric closure of $X$. 
Statement (2) is clear because $f$ takes paths of radius $<\delta$ to paths of radius $<\epsilon$.

In (3) uniform continuity implies Cauchy sequences in $X$ are taken to Cauchy sequences in $Y$, so the map extends to a map of completions. If the image of a Cauchy sequence converges in $Y$ then by properness the inverse image of the closure is compact in $X$. But a Cauchy sequence in a compact space converges. Thus if the original sequence does not converge in $X$, neither does the image, and so completion points in $X$ map to completion points in $Y$.

4.1.3 Geometric modules and morphisms. The setting is a "coefficient map" $p: E \rightarrow X$ with $X$ a metric space. Distances are measured in $X$, while $E$ serves to record fundamental group data.

A geometric $R$-module on $p: E \rightarrow X$ is a free module with basis $S$, and a function $s: S \rightarrow E$.

A morphism of geometric modules is a formal linear combination of paths in $E$. Specifically $f: R\left[S_{1}\right] \rightarrow R\left[S_{2}\right]$ is of the form $\Sigma_{i} r_{i} \gamma_{i}$, where

(1) $r_{i} \in R$;

(2) $\gamma_{i}$ consists of points $\gamma_{i}(0) \in S_{1}, \gamma_{i}(1) \in S_{2}$, and a path $\gamma:[0,1] \rightarrow E$ from the image of $\gamma_{i}(0)$ to the image of $\gamma_{i}(1)$ such that

(3) only finitely many of the $\gamma_{i}$ begin at any given element of $S_{1}$.

We consider two morphisms to be the same if they differ by the operations

(1) omit a path with zero coefficient, or

(2) if a path occurs multiple times, replace with a single copy and add the coefficients.

Note that a geometric morphism induces a homomorphism on the free modules generated by the basis sets. In some cases the geometric morphism can be essentially reconstructed from the homomorphism, but even in these cases the geometric version works better in constructions.

Geometric morphisms can be composed in the evident way: compose paths when possible, and multiply coefficients. This composition can be made associative by interpreting "path" to mean a map of $[0, t]$ for some $t \geq 0$ rather than restricting to $[0,1]$. Composed paths are then defined on longer intervals, and do not require reparameterization to get back to $[0,1]$. 
A geometric morphism is said to have radius less than $\epsilon$ if all paths with nonzero coefficients have images in $X$ of radius $<\epsilon$.

Morphism radius is subadditive in compositions: if $f: A \rightarrow B$ has radius $\leq \epsilon$ and $g: B \rightarrow C$ has radius $\leq \delta$ then $g f$ has radius $\leq \epsilon+\delta$. In particular $\epsilon$ morphisms do not form a category because compositions are usually bigger than $\epsilon$.

If $Y \subset X$ then the restriction $f \mid Y$ consists of the paths of $f$ that lie in $p^{-1}(Y)$. Restriction is functorial in the sense that composition of restrictions is the restriction of the composition.

4.1.4 Homotopy of morphisms. A homotopy of a geometric morphism $\Sigma r_{i} \gamma_{i}$ consists of homotopies (rel ends) of all the paths $\gamma_{i}$. A homotopy determines another morphism by using the same coefficients and the paths at the end of the homotopies. Two morphisms are homotopic if (after combinations and omission of paths with zero coefficient) they can be related in this way.

A homotopy of geometric morphisms has radius less than $\epsilon$ if each of the homotopies individually has radius less than $\epsilon$. Generally a homotopy $Y \times I \rightarrow X$ has radius less than $\epsilon$ if the restriction to each $\operatorname{arc}\{y\} \times I$ has radius $<\epsilon$. Explicitly this means $h: Y \times I \rightarrow X$ satisfies $d(h(y, s), h(y, t))<\epsilon$ for all $y \in Y$, $s, t \in I$

Note that a $\delta$ homotopy of an $\epsilon$ morphism gives a morphism of radius less than $\epsilon+2 \delta$. Reversing an $\epsilon$ homotopy from $f_{0}$ to $f_{1}$ gives an $\epsilon$ homotopy from $f_{1}$ to $f_{0}$. Homotopies can be composed, and radii add. The additivity means " $\epsilon$ homotopy" is not an equivalence relation, and we cannot work with "homotopy classes". As with the failure to be a category this requires us to be more direct and explicit in constructions.

The restriction of a homotopy to $Y \subset X$ consists of all the individual path homotopies that lie in $Y$. Restriction and homotopy do not play well together, but homotopies of radius $<\epsilon$ give predictable results over the reduced set $Y^{-\epsilon}$.

4.1.5 Uncontrolled morphisms. Note that if no size conditions are imposed then morphisms on $E \rightarrow X$ are a category, and homotopy does give an equivalence relation. In this case $X$ becomes irrelevant and choice of a basepoint gives an equivalence between homotopy classes of $R$-morphisms and homomorphisms over the ring $R\left[\pi_{1} E\right]$ [Quinn 6]. Relaxing control therefore gives a map 
of controlled algebra into a classical algebraic setting.

4.1.6 Main example: geometric cellular chains. Let $X$ be a CW complex with skeleta $X^{(n)}$. We suppose $X$ is slightly generic in the sense that attaching maps for $n+1$-cells $S^{n} \rightarrow X^{(n)}$ are differentiable with invertible derivative at points mapping to centers of $n$-cells. This means these points are isolated, and the sign of the derivative associates \pm 1 to each one. An arbitrary CW complex can be made generic by arbitrarily small perturbations of the attaching maps, so the conclusions apply generally.

For each integer $n \geq 0$ define a geometric $Z$-complex $C_{n}$ by taking the basis to be the centers of the $n$-cells.

Define a geometric morphism $\partial: C_{n} \rightarrow C_{n-1}$ by: the paths from the center of an $n$-cell are rays to points on $S^{n-1}$ that map to centers of $n$-1-cells under the attaching map. The coefficient on a path is the sign of the determinant of the derivative of the attaching map at the endpoint. The groups and homomorphisms obtained from these geometric objects are exactly the usual cellular chain complex for $X$. However in the geometric version $\partial^{2}$ is plainly not trivial.

Lemma. The composition $\partial^{2}$ in this complex is homotopic to 0 . If each cell has radius $<\epsilon$ then $\partial$ has radius $<\epsilon$ and the nullhomotopy has radius $<2 \epsilon$.

Indication of proof. (See [Quinn 6] for details.) Suppose $\sigma$ is an $n$-cell and $\tau$ an $n-2$-cell. The usual geometric proof that algebraically $\partial^{2}=0$ shows the composed paths from center of $\sigma$ to center of $\tau$ occur in pairs with opposite signs. It also shows that these pairs are homotopic, by a homotopy that is very close to the union of $\sigma, \tau$, and the intermediate $n-1$-cells. These homotopies give a homotopy of $\partial^{2}$ to a morphism with half as many paths, and coefficient $1+(-1)$ on each one. We can omit all these to get the 0 geometric morphism. The estimates follow from the locations of the paths and homotopies.

4.1.7 Controlled chain notions. The definition abstracts what we would get by restricting the example above to an open set. Alternatively we "add control" to the usual definitions following what will become a familiar pattern: modules become geometric modules, homomorphism become geometric morphisms, identities ( such as $\partial^{2}=0$ ) become homotopies that are only assumed to exist, and identities are allowed to fail near the metric frontier. In the following we fix 
$p: E \rightarrow X$ and $\epsilon>0$.

An $\epsilon$ chain complex over $p$ consists of

(1) geometric modules $C^{i}$ for $i$ nonnegative integers;

(2) geometric $R$-morphisms $c$ : $C^{i} \rightarrow C^{i-1}$ with radius $<\epsilon$; and

(3) there exist (for each $i$ ) a homotopy of radius $<\epsilon$ from $c^{2}$ to a morphism $C^{i} \rightarrow C^{i-2}$ that is trivial on basis elements outside $\operatorname{Fr}^{3 \epsilon} X$.

Similarly an $\epsilon$ chain map $f:(C, c) \rightarrow(D, d)$ consists of

(1) for each $i$ a geometric morphism $f: C^{i} \rightarrow D^{i}$ of radius $<\epsilon$;

(2) for each $i$ there exist homotopies of radius $<\epsilon$ from $d f: C^{i} \rightarrow D^{i-1}$ to morphisms that agree with $f c$ on basis elements outside $\operatorname{Fr}^{\epsilon} X$.

Finally an $\epsilon$ chain contraction $\xi$ for a complex $(C, c)$ is

(1) geometric morphisms $\xi: C^{i} \rightarrow C^{i+1}$ of radius $<\epsilon$ so that

(2) there exist $\epsilon$ homotopies starting with $c \xi+\xi c: C^{i} \rightarrow C^{i}$ and going to morphisms that are the identity on basis elements not in $\mathrm{Fr}^{3 \epsilon}$.

4.1.8 $\epsilon$ isomorphisms. The definition here differs from the usual one by allowing misbehavior near the metric frontier.

Suppose $f: A \rightarrow B$ is a geometric morphism over $p: E \rightarrow X$. $f$ is an $\epsilon$ isomorphism if

(1) $f$ has radius $<\epsilon$;

(2) there is $f^{-}: B \rightarrow A$ with radius $<\epsilon$ such that

(3) there are $\epsilon$ homotopies $h_{A}, h_{B}$ starting with $f^{-} f$ and $f f^{-}$respectively and ending with morphisms that are identities when restricted to $A \mid(X-$ $\left.\operatorname{Fr}^{3 \epsilon} X\right), B \mid\left(X-\operatorname{Fr}^{3 \epsilon} X\right)$ respectively.

Lemma. Suppose $f: A \rightarrow B$ is an $\epsilon$ isomorphism over $p: E \rightarrow X$, and $U \subset X$ is open. Then the restriction $f|U: A| U \rightarrow B \mid U$ is an $\epsilon$ isomorphism over $U$.

Previous versions of this lemma asserted that $f \mid U$ is an "isomorphism over $U^{-3 \epsilon}$ ". This however depends on the situation of $Y$ in $X$ while the lemma above uses $U-\operatorname{Fr}^{3 \epsilon} U$, which is intrinsic to $U$.

Let $f^{-}, h_{A}, h_{B}$ be data showing that $f$ is an $\epsilon$ isomorphism on $X$. We claim the restrictions to $U$ give data showing $f \mid U$ is an $\epsilon$ isomorphism on $U$. Fix a basis element $x \in A$, then the first assertion is that $f(x)=(f \mid U)(x)$ unless $x \in \operatorname{Fr}^{\epsilon} U$. 
If the two morphisms differ on $x$ then there is a path $\gamma$ of $f$ that starts at $x$ and intersects $X-U$. Let $t_{0}$ be the minimum of $\{t \mid \gamma(t) \notin U\}$. Since $U$ is open this point is in the closure of $U$ but not in $U$ itself. It thus gives a point in the metric frontier. Since the path has radius $<\epsilon, x$ is in $\operatorname{Fr}^{\epsilon} U$.

Similarly $f^{-} \mid U$ equals $f^{-}$except within $\epsilon$ of the frontier. Composing we see that $\left(f^{-} \mid U\right)(f \mid U)$ is equal to $f^{-} f$ on $A \mid\left(U-\mathrm{Fr}^{2 \epsilon} U\right)$.

Finally the same argument shows that the homotopy $h_{A} \mid U$ is equal to $h_{A}$ on any path that does not intersect $\operatorname{Fr}^{\epsilon} U$. Therefore on $A \mid\left(U-\operatorname{Fr}^{3 \epsilon} U\right)$ the morphisms, composition, and homotopy are all unchanged. By hypothesis the original homotopy ends with the identity over $X-\operatorname{Fr}^{3 \epsilon} X \supset\left(U-\operatorname{Fr}^{3 \epsilon} U\right)$. This gives the conclusion needed for the lemma.

\subsection{Triangularity.}

4.2.1 Diagonal and triangular morphisms. The main modification in the uncontrolled definition is to relax conditions near the metric frontier.

Suppose $A, B$ are geometric modules over $E \rightarrow X$ and $U$ is a subgroup of the units of $R$ ( in this paper $\{1\}$ or $\{ \pm 1\}$ ).

A geometric morphism $d: A \rightarrow B$ is $\epsilon, U$ diagonal over $X$ if there is at most one path beginning on each basis element of $A$, there is such a path beginning at $x$ if $x \notin \operatorname{Fr}^{\epsilon} X$, the endpoints of these paths are distinct, the coefficients are elements of the subgroup $U$, and the paths all have radius $<\epsilon$.

Compositions of $\epsilon$ diagonal morphisms are $2 \epsilon$ diagonal. More generally the composition of an $\epsilon$ and a $\delta$ diagonal morphism is $\epsilon+\delta$ diagonal.

A geometric morphism is $\epsilon$ triangular if it is an $\epsilon$ morphism and also triangular in the uncontrolled sense. It follows for instance that the composition of two $\epsilon$ triangular morphisms is $\epsilon$ triangular. Additional leverage comes from hypotheses on the partial order rather than modification of "triangularity."

4.2.2 $\epsilon$-bounded partial orders. Suppose $X$ is a metric space and $S \rightarrow X$ is a function. A partial order on $S$ is $\epsilon$ bounded if for each $s \in S$ there is $n$ so that any increasing chain starting with $s$ has length bounded by $n$ and its image in $X$ lies in the $\epsilon$ ball about the image of $s$.

The following is the analog of Lemma 3.2.4:

Lemma. Suppose $f: A \rightarrow B$ is $\epsilon$ triangular with respect to an $\epsilon$ bounded partial 
order on the base of $B$. Then

(1) the diagonal part of $f$ is well-defined independently of the choice of such a partial order;

(2) $f$ has a $3 \epsilon$ triangular inverse;

(3) if $U \subset X$ is open then the restriction to $U$ is $\epsilon$ triangular over $U$;

(4) if $g: B \rightarrow C$ is $\delta$ triangular with respect to the same partial order on $B$ then $g f$ is $\epsilon+\delta$ triangular;

(5) there is a factorization $f=\cdots\left(1+\alpha_{n}\right) \cdots\left(1+\alpha_{1}\right) \operatorname{diag}(f)$ with $\alpha_{i}$ increasing, $\alpha_{i} \alpha_{j}=0$ if $j \leq i$, and starting at any basis element the composition is finite with paths of length $<\epsilon$.

The proof is a straightforward extension of the proof of 3.2.4. For instance if we write $d+u=\left(1+u d^{-1}\right) d$ then the inverse of the first factor is $\sum_{i=0}^{\infty}\left(-u d^{-1}\right)$ and the inverse for the composition is $d \sum_{i=0}^{\infty}\left(-u d^{-1}\right)$. Note the sum makes sense because the partial order is locally bounded. The terms in the sum are arbitrarily long compositions but have paths of radius $<3 \epsilon$. This is because they are compositions of paths of radius $<\epsilon$ joining points that lie within $\epsilon$ of their starting point, since the partial order is $\epsilon$ bounded.

Similarly the factorization is obtained as in 3.2.4. Again we observe the partial order condition shows the factorization exists, but the construction does not depend on the partial order.

4.2.3 Examples. These illustrate the force of the $\epsilon$ hypothesis on the partial order. Let $X$ be the real line, and $A_{i, j}$ the geometric module generated by the integers $i, \ldots, j$. Define $u: A_{i, j} \rightarrow A_{i, j}$ by: if $i \leq k<j$ then $u(k)$ is the segment from $k$ to $k+1$ with coefficient 1 , and $u(j)=0$. Then id $+u$ is triangular with respect to the standard linear order. It has radius $<1+\delta$ for any $\delta>0$. It is an isomorphism. However the inverse has radius $j-i$.

By mapping this example into a space $X$ we get examples of $\epsilon$ morphisms that are isomorphisms and triangular in the uncontrolled sense, but whose inverse has radius arbitrarily large up to the diameter of $X$.

Setting $i=-\infty, j=\infty$ (so $A$ is generated by all the integers, and $u$ is the upward shift) we get $\epsilon$ morphisms triangular in the uncontrolled sense and for which $d(=\mathrm{id})$ is an isomorphism, but $d+u$ is not even an uncontrolled isomorphism. 
4.2.4 Cancellation. Suppose $C$ is an $\epsilon$ chain complex and $\xi$ an $\epsilon$ chain contraction. As in as in 3.1.1 we say $\xi$ cancels the complement of a based subcomplex $\hat{C}$ if there is a based decomposition $C \perp \hat{C}=D \oplus \bar{D}$ such that the $C \rightarrow C \perp \hat{C}$ component of the boundary homomorphism has the form

$$
\left(\begin{array}{ccc}
\hat{\xi} & u & v \\
0 & 0 & \delta \\
0 & 0 & 0
\end{array}\right): \hat{C} \oplus D \oplus \bar{D} \longrightarrow \hat{C} \oplus D \oplus \bar{D}
$$

with $\delta$ a $\pm 1 \epsilon$ triangular morphism.

4.3 Definition of $K_{1}^{l f}(X ; p, R, \epsilon)$. In this section we define a space of $\epsilon$ controlled complexes over $p: E \rightarrow X$, essentially by adding " $\epsilon$ geometric" and a local finiteness hypothesis to everything in definition 3.4.1. Basic naturality properties (those not needing spacial localization) are also given in this section: restrictions in 4.3.2, functional images in 4.3.3, and dependence on the reference map in 4.3.4.

4.3.1 Simplices in $K_{1}^{l f}$. Fix $\epsilon>0$. An $n$-simplex of $K_{1}^{l f}(X ; p, R, \epsilon)$ consists of:

(1) $\epsilon$ chain complexes $C_{i}$ over $p$ for $0 \leq i \leq n$ so that the basis elements have no points of accumulation in the metric completion;

(2) contractions $\xi_{i}$ for $C_{i}$ so that $\left(\xi_{i}\right)^{k}$ has radius $<\epsilon$ for all $k$;

(3) for $i<j$ an $\epsilon$ chain map $c_{i, j}: C_{i} \rightarrow C_{j}$;

(4) there exist $\epsilon$ bounded partial orders on the bases of the $C_{i}$ in each degree so that

i) the chain maps $c_{i, j}$ are $\epsilon$ triangular isomorphisms onto their images in each degree, and if $i<j<k$ then the underlying basis functions for $c_{i, k}$ and $c_{j, k} c_{i, j}$ are equal on elements not taken to 0 by either;

ii) in each degree, basis elements in $C_{j}$ not in the image of $c_{i, j}$ and outside $\operatorname{Fr}^{\epsilon} X$ preceed those in the image of $c_{i, j}$; and

iii) the contraction $\xi_{j}$ cancels the complements of the images of $c_{i, j}$.

As in 3.4 we define faces of $K_{1}$ simplices by omitting one of the complexes. The collection of all such simplices defines a simplicial complex (or a $\Delta$-set), and this (or its geometric realization) is defined to be $K_{1}^{l f}(X ; p, R, \epsilon)$.

4.3.2 Restrictions. Suppose $U \subset X$ is an open set. All the definitions have been arranged so restrictions of $\epsilon$ objects to $U$ give $\epsilon$ objects on $U$. Therefore we have contrived: 
Lemma. If $U \subset X$ is open then restriction defines a natural map

$$
K_{1}^{l f}(X ; p, R, \epsilon) \rightarrow K_{1}^{l f}(U ; p, R, \epsilon)
$$

This is an important part of the structure used to identify the limit as homology.

4.3.3 Functional images. Suppose

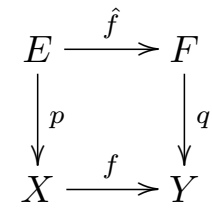

is a commutative diagram with $X, Y$ metric spaces. Applying $(f, \hat{f})$ to geometric gadgets over $p$ gives geometric gadgets over $q$. A size estimate implies it takes controlled gadgets to controlled gadgets:

Lemma. Suppose $f: X \rightarrow Y$ is a proper map, extends to a proper map of completions, and $d\left(x, x^{\prime}\right)<\delta$ implies $d(f(x), f(y))<\epsilon$. Then composition induces a natural map

$$
K_{1}^{l f}(X ; p, R, \delta) \stackrel{f_{*}}{\longrightarrow} K_{1}^{l f}(Y ; q, R, \epsilon)
$$

The properness is needed to see that the frontier of $X$ maps to that of $Y$, and that images of bases do not acquire accumulation points in $Y$. Otherwise this should be clear.

4.3.4 Dependence on the data. Definitions of geometric algebra over $p: E \rightarrow X$ do not use very much of $E$ and $X$. All the paths and homotopies essentially lie in the 2-skeleton, so other data with similar 2-skeleta should have the same algebra and $K$-theory. The next definition and lemma make this precise.

Definition. a commutative diagram

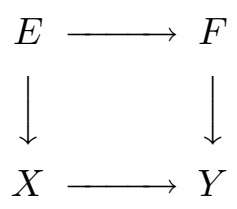


is $(\delta, 1)$-connected if for every relative 2-complex $(K, L)$ and commutative diagram

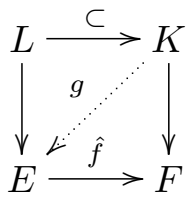

there is a map $g$ (dotted arrow) so that the upper triangle commutes, the lower triangle commutes up to $\delta$, and on the 1-skeleton of $K$ it $\delta$ homotopy commutes $(\delta$ measured in $X)$. Previous versions [Quinn 1,2] did not include the 1-skeleton homotopy condition and we had to reconstruct it using a $(\delta, 1)$-connectedness hypothesis on $X$ itself. Putting the condition in this definition makes the following easier and more general:

Lemma. Suppose $\epsilon, \delta>0$ and $\hat{f}: p \rightarrow q$ is a $(\delta, 1)$-connected map of spaces over an embedding $X \subset Y$. Then there is a map $f^{*}$ as shown that makes the diagram homotopy commute:

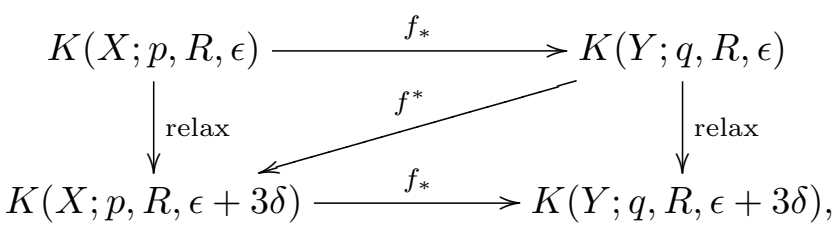

where $K$ is short for $K_{1}^{l f}$.

We have assumed $X$ is a subspace (i.e. $X \rightarrow Y$ is an isometry) to avoid having to spell out metric conditions on the map. Note that $\epsilon$ algebra only uses an $\epsilon$ dense 2-skeleton of $X$. In the limit as $\epsilon \rightarrow 0$ all of $X$ gets used, but 1-connectedness is still sufficient at the $E$ level.

Proof. Begin with a geometric module $A$ over $q$. Apply 1 -connectedness with $K$ the basis of $A$ and $L$ empty. This gives a $\delta$ homotopy of the basis to the image of a factorization through $\hat{f}$. Define $f^{*} A$ to be a module over $p$ obtained this way, then the basis homotopy gives a basis-preserving isomorphism $\hat{f}_{*} f^{*} A \rightarrow A$ of radius $<\delta$.

Now suppose $c: A \rightarrow B$ is a geometric morphism of modules over $q$ with radius $<\epsilon$. Composing with the basis-preserving isomorphisms above gives a 
morphism $\hat{f}_{*} f^{*} A \rightarrow \hat{f}_{*} f^{*} B$. Apply the 1-connectedness hypothesis again, this time with $K$ the paths in this morphism and $L$ the bases of $f^{*} A$ and $f^{*} B$. Use the resulting paths in $E$ and the coefficients in $c$ to define $f^{*} c: f^{*} A \rightarrow f^{*} B$. The size constraint on the lift shows these paths have radius $<\epsilon+\delta$. The homotopy part of 1-connectedness gives $\delta$ homotopies from the $\hat{f}$ images of these paths to the paths in the morphism, and we conclude that $\hat{f}_{*} f^{*} c$ is $\delta$ homotopic to $c$. Moreover since there is a one-to-one correspondence between paths in the two morphisms, $f^{*} c$ is triangular if $c$ is.

Finally we consider relations. We need that if the composition $A \stackrel{a}{\rightarrow} B \stackrel{b}{\rightarrow} C$ is $\epsilon$ homotopic to $c: A \rightarrow C$ then $\left(f^{*} b\right)\left(f^{*} a\right)$ is $\epsilon+3 \delta$ homotopic to $f^{*} c$. First note we get an $\epsilon+2 \delta$ homotopy of images $\left(\hat{f}_{*} f^{*} b\right)\left(\hat{f}_{*} f^{*} a\right)$ and $\hat{f}_{*} f^{*} c$ by composing the homotopies from these to $b a$ and $c$ with the given homotopy from $g a$ to $c$. Apply 1-connectedness with $K$ the domain of these homotopies (a union of copies of $I \times I)$ and $L$ the boundaries. We conclude there are homotopies in $E$ whose images in $F$ are within $\delta$ of the input data. The distance estimate shows these homotopies have radius $<\epsilon+3 \delta$, as required. There is no conclusion about the images of these lifts being homotopic to the input data, but we do not need it: relations require only existence.

The fact that homotopy relations lift shows that if $c$ is an $\epsilon$ isomorphism or triangular isomorphism then $f^{*} c$ is an $\epsilon+3 \delta$ isomorphism or triangular isomorphism. similarly chain complexes lift to chain complexes, chain maps to chain maps, etc. This shows $f^{*}$ defines $K_{1}^{l f}(X ; q, r, \epsilon) \rightarrow K_{1}^{l f}(X ; q, r, \epsilon+3 \delta)$ as required. The basis-preserving maps $\hat{f}_{*} f * A \rightarrow A$ constructed in the first paragraph give the homotopy making the lower triangle in the diagram in the lemma commute. If $B$ is geometric over $p$ then a similar construction gives a basis-preserving isomorphism $B \rightarrow f^{*} \hat{f}_{*} B$, and this gives a homotopy making the upper triangle commute.

This completes the proof of the lemma.

4.4 The limit $K_{1}^{l f}(X ; p, R)$. The main object of the paper is defined in 4.4 .1 as the inverse limit of the $\epsilon$ spaces of the previous section. Functoriality is described in 4.4.2.

4.4.1 Definition of $K_{1}^{l f}$. If $\epsilon>\delta>0$ then relaxing control gives an inclusion $K_{1}^{l f}(X ; p, R, \delta) \subset K_{1}^{l f}(X ; p, R, \epsilon)$. This defines an inverse system as $\epsilon \rightarrow 0$, and 
as indicated in 2.1.4 we define

$$
K_{1}^{l f}(X ; p, R)=\operatorname{holim}_{\epsilon \rightarrow 0} K_{1}^{l f}(X ; p, R, \epsilon)
$$

Homotopy inverse limits (the "holim" in the definition) are discussed in 2.1.4. Using the path model for linearly ordered homotopy limits we can describe these as maps $(0,1] \rightarrow K_{1}^{l f}(X ; p, R, \gamma)$, some $\gamma$, that "converge in $X$ " in the limit $t \rightarrow 0$. More explicitly this means a triangulation of $(0,1]$ (usually infinite near 0 ) and a map so that for every $\epsilon>0$ there is $\delta>0$ so that on $(0, \delta]$ the image lies in $K_{1}^{l f}(X ; p, R, \epsilon)$.

The naturality of the $\epsilon$ versions passes to the limit:

4.4.2 Proposition. $K_{1}^{l f}(X ; p, R)$ has natural restrictions to open sets, and is functorial with respect to morphisms $(p: E \rightarrow X) \longrightarrow(q: F \rightarrow Y)$ over proper uniformly continuous $X \rightarrow Y$. Finally if $X=Y$ and $E \rightarrow F\left(\delta_{i}, 1\right)$-connected over $X$ for all $\delta_{i}>0$ then the induced map on $K_{1}^{l f}$ is a homotopy equivalence.

Note the uniform continuity condition on naturality is too restrictive for the characterization theorem. It is extended to naturality over all proper maps in the next section.

\section{SPACIAL LOCALIZATION AND THE AXIOMS}

Spacial localization refers to factorization of controlled activity, usually homotopies, into pieces constant over various pieces of the control space. For instance the cancellation of inverses is a canonical nullhomotopy of the map that takes a complex to the complex plus its suspension. In 5.1 the controlled version is "localized" to a subset $Y$ in the sense that the homotopy is described as the composition of two homotopies, one that keeps things constant over $Y$ and cancels away from $Y$, and another that finished the cancellation over $Y$. This depends on special properties of triangular isomorphisms so is less formal than the properties developed in $\S 4$. Section 5.2 extends this to a "skew inverse" version: a $K_{1}$ morphism $B \rightarrow A$ determines a cancellation of $A \oplus S B$. Again this can be localized to a subset. Finally these are used in $\S 5.3$ to localize general homotopies.

The constructions of $\S \S 5.1-5.3$ are used to verify the homology axioms of 6.1 for $K$-theory. The homotopy axiom is verified in 5.4 using the simplest case of 
cancellation. Localization of homotopies is used to verify the exactness axiom in 5.4 and independence of the metric in 5.5. The stability theorem 2.3.1 is also proved with these tools.

5.1 Standard inverses. The cancellation of inverses is a canonical nullhomotopy $H:$ id $\oplus S \sim 0$ as maps from $K_{1}$ to itself, see $\S 3.5$. The objective here is to show that the process preserves $\epsilon$ control in an appropriate sense, and to localize it: factor it into a part that cancels the sum away from $Y$ but doesn't change it over $Y$, and a part that finishes the cancellation over $Y$.

5.1.1 Proposition. Suppose $X, p, R$ as usual, $X \supset Y$, and $\epsilon>0$ is fixed. Then there are

(1) a canonical map $T: K_{1}^{l f}(X ; p, R, \epsilon)^{(1)} \rightarrow K_{1}^{l f}(X ; p, R, 7 \epsilon)$, where the superscript (1) indicates the first derived subdivision;

(2) canonical homotopies $H_{1}:$ id $\oplus S \sim T$ and $H_{2}: T \sim 0$; and

(3) a canonical homotopy $G: H_{1} H_{2} \sim H$, where $H$ is the standard cancellation (i.e. $\mathrm{H}_{2}$ for the case $Y$ empty).

These satisfy:

(1) restricted to $Y, H_{1}$ is constant equal to the identity, $H_{2}=H$ and $G$ is constant;

(2) restricted to $X-Y^{20 \epsilon}, H_{1}=H, H_{2}$ is constant equal to 0 , and $G$ is constant;

(3) If $Y^{50 \epsilon}-Y^{-25 \epsilon} \subset U$ and $U$ is open in $X$ then $T, H_{i}$ and $G$ are natural with respect to restriction to $(U, Y \cap U)$; and

(4) on $K_{1}^{l f}(X ; p, R, \delta) H_{i}$ and $G$ have radius $<7 \delta$.

The $Y$-dependent parts of the construction are actually determined by data over $Y^{20 \epsilon}-Y$. A more generous region is used in (3) to avoid ambiguity with a detail of the definition of $K$, namely that structural hypotheses on $\gamma$ objects are allowed to fail within $3 \gamma$ of the metric frontier of the space. This means for $\gamma=7 \epsilon$ on $Y^{50 \epsilon}-Y^{-25 \epsilon}$ there may be some question about what happens outside $Y^{29 \epsilon}-Y^{-4 \epsilon}$, but this is safely away from $Y^{20 \epsilon}-Y$.

5.1.2 Notation and outline of the proof. Fix $X, p, R$ and shorten $K_{1}^{l f}(X ; p, R, \epsilon)$ to $K_{1}(\epsilon)$. The constructions are canonical (after choice of $Y$ and $\epsilon$ ) so need only 
be described for a typical simplex of $K_{1}(\epsilon)$. We write an $n$-simplex as 4-tuple $\left(C, c, \xi, c_{i, j}\right)$, where $C$ is a sequence of graded modules $C_{i}$ for $0 \leq i \leq n, c_{i}$ is a boundary morphism for $C_{i}, \xi_{i}$ is a contraction for the complex $\left(C_{i}, c_{i}\right)$, and $c_{i, j}: C_{i} \rightarrow C_{j}$ are chain maps, all in the controlled geometric senses defined in Section 4 .

In these terms the starting point, $(\mathrm{id} \oplus S)(C)$ is given by

$$
\left(C \oplus S C,\left(\begin{array}{cc}
c & 0 \\
0 & -c
\end{array}\right),\left(\begin{array}{cc}
\xi & 0 \\
0 & -\xi
\end{array}\right),\left(\begin{array}{cc}
c_{i, j} & 0 \\
0 & c_{i, j}
\end{array}\right)\right) .
$$

Recall that the negative signs in the $S C$ component of the boundary and contraction come from the sign conventions of 3.1.3. Namely, the suspension functor changes signs of maps by $(-1)^{\text {degree }}$, and the boundary and contraction have degrees -1 and 1 respectively. There is no sign change in the chain map piece because chain maps have degree 0 .

The construction proceeds in three steps, each of which produces $K_{1}$ morphisms. The final homotopies are obtained by subdividing morphisms as described in 3.4.4. The first two steps standardize structure of objects but do not change underlying graded modules. The third step truncates modules. We show how to factor each step, and assemble the three steps by arranging that factorizations of the pieces can be commuted. The first derived subdivision arises in factoring the truncation step.

5.1.3 Morphism to cones. This is an elaboration of the fact that $\left(\begin{array}{cc}1 & -\xi \\ 0 & 1\end{array}\right)$ gives a chain isomorphism from $C \oplus S C$ to the mapping cone of the identity.

Conjugating the starting data $(*)$ with $\left(\begin{array}{cc}1 & -\xi \\ 0 & 1\end{array}\right)$ gives a morphism to the simplex with data

$$
\left(C \oplus S C,\left(\begin{array}{cc}
c \xi c+c \xi \\
0 & -c
\end{array}\right),\left(\begin{array}{cc}
\xi & 2 \xi^{2} \\
0 & -\xi
\end{array}\right),\left(\begin{array}{cc}
c_{i, j} & c_{i, j} \xi_{i}-\xi_{j} c_{i, j} \\
0 & c_{i, j}
\end{array}\right)\right) .
$$

There is a homotopy of this to

$$
\left(C \oplus S C,\left(\begin{array}{cc}
c & 1 \\
0 & -c
\end{array}\right),\left(\begin{array}{cc}
\xi & 0 \\
0 & -\xi
\end{array}\right),\left(\begin{array}{cc}
c_{i, j} & 0 \\
0 & c_{i, j}
\end{array}\right)\right)
$$

in the following sense:

(1) By the hypothesis that $\xi$ is a contraction there is an $\epsilon$ homotopy of geometric morphisms $\xi c+c \xi \sim 1$. 
(2) The degree-2 homomorphism $\left(\begin{array}{cc}0 & 2 \xi^{3} \\ 0 & 0\end{array}\right)$ is a chain homotopy from $\left(\begin{array}{cc}\xi & 2 \xi^{2} \\ 0 & -\xi\end{array}\right)$ to $\left(\begin{array}{cc}\xi & 0 \\ 0 & -\xi\end{array}\right)$ (after homotopy of geometric morphisms), and

(3) $\left(\begin{array}{cc}0 & \xi_{j} c_{i, j} \xi_{i} \\ 0 & 0\end{array}\right)$ is a chain homotopy from $\left(\begin{array}{cc}c_{i, j} & \xi_{j} c_{i, j}+c_{i, j} \xi_{i} \\ 0 & c_{i, j}\end{array}\right)$ to $\left(\begin{array}{cc}c_{i, j} & 0 \\ 0 & c_{i, j}\end{array}\right)$, again after homotopy of geometric morphisms.

If the original simplex has radius $\leq \delta$ then all of these homotopies have radius less than $5 \delta$. The largest potential excursions come from using the contraction identity to simplify the result of applying the boundary to the $2 \xi^{3}$ term in the homotopy of the contraction.

We claim the morphism $\left(\begin{array}{cc}1 & -\xi \\ 0 & 1\end{array}\right)$ gives a $K_{1}(7 \epsilon)$ morphism from the starting data $(*)$ to $(* *)$. The content of this assertion is that there are $7 \epsilon$-bounded partial orders in which the chain maps and morphism are triangular, and complements of images cancel. These partial orders are obtained from partial orders assumed to exist in the original simplex by a modification of "shuffling the image partial orders", see the proof of 3.5.1. In detail, the basis of $C_{i} \oplus S C i$ in degree $k$ is the union base $\left(C_{i}^{k}\right) \cup \operatorname{base}\left(C_{i}^{k-1}\right)$. On each subset the partial order is the given one. Suppose $s \in \operatorname{base}\left(C_{i}^{k}\right)$ and $t \in \operatorname{base}\left(C_{i}^{k-1}\right)$. If they are comparable then $s>t$. Define them to be comparable if

(1) the distance between them is less than $5 \epsilon$, and

(2) for every $j<i$, if $t \in \operatorname{im}\left(S C_{j}\right)$ then $s \in \operatorname{im}\left(C_{j}\right)$.

An increasing chain in this partial order consists of a chain in $S C$ followed by a chain in $C$. The sub-chains have size bounded by $\epsilon$ and the beginning of the second is within $5 \epsilon$ of the end of the first. Thus the maximum size is $7 \epsilon$. The total length is bounded because the lengths of the subchains are. This therefore defines a $7 \epsilon$ bounded partial order. $\left(\begin{array}{cc}1 & -\xi \\ 0 & 1\end{array}\right)$ and the homotopies used to modify the structure are all triangular in this partial order because the maps on the individual summands are, they preserve images (see 3.3), and the inter-summand maps have size $<5 \epsilon$.

We now localize this. Let $U_{1}$ be a subset of $X$ (to be specified later in terms of $Y$ ). Denote by $\rho$ the projection of a geometric module to the submodule supported by $U_{1}$. Denote projection to the complement of $U_{1}$ by $(1-\rho)$. This is a slight abuse of notation since $(1-\rho)$ technically defines a homomorphism 
homotopic to this projection. Factor the basic morphism by

$$
\left(\begin{array}{cc}
1 & -\xi \\
0 & 1
\end{array}\right)=\left(\begin{array}{cc}
1 & -\xi(1-\rho) \\
0 & 1
\end{array}\right)\left(\begin{array}{cc}
1 & -\xi(\rho) \\
0 & 1
\end{array}\right)
$$

We extend this to a factorization of the whole $K_{1}$ morphism. Conjugating by the first (right) factor takes the starting data to

$$
\left(C \oplus S C,\left(\begin{array}{cc}
c \xi \rho c+c \xi \rho \\
0 & -c
\end{array}\right),\left(\begin{array}{cc}
\xi \rho \rho \xi+\xi^{2} \rho \\
0 & -\xi
\end{array}\right),\left(\begin{array}{cc}
c_{i, j} & c_{i, j} \xi_{i} \rho-\xi_{j} \rho c_{i, j} \\
0 & c_{i, j}
\end{array}\right)\right) .
$$

Modify the contraction and chain maps by homotopies $\left(\begin{array}{cc}0 & 2(\xi)^{3} \rho \\ 0 & 0\end{array}\right)$ and $\left(\begin{array}{cc}0 & \xi_{j} c_{i, j} \xi_{i} \rho \\ 0 & 0\end{array}\right)$ respectively. Finally note that the $\epsilon$ homotopy of homomorphisms $\xi c+c \xi \sim 1$ provides a homotopy of $\xi \rho c+c \xi \rho$ to a morphism that is 1 over $U_{1}^{-\epsilon}$ and is constant over $X-U^{\epsilon}$ where $\xi \rho c+c \xi \rho=0$. Putting these together gives a simplex structure on the modules $C \oplus S C$ that agrees with the cone structure over $U_{1}^{-5 \epsilon}$ and the sum structure over $X-U_{1}^{5 \epsilon}$. The homomorphism $\left(\begin{array}{cc}1 & -\xi(\rho) \\ 0 & 1\end{array}\right)$ provides a $K_{1}$ morphism from the sum structure to this, and is the identity over $X-U_{1}^{5 \epsilon}$.

This defines the first factor. The second factor is obtained similarly: consider $\left(\begin{array}{cc}1 & -\xi(1-\rho) \\ 0 & 1\end{array}\right)$ as a map from the object just constructed to the object with structure obtained by conjugating. Modify this by the rest of the chain homotopies, namely $\left(\begin{array}{cc}0 & 2(\xi)^{3}(1-\rho) \\ 0 & 0\end{array}\right)$ and $\left(\begin{array}{cc}0 & \xi_{j} c_{i, j} \xi_{i}(1-\rho) \\ 0 & 0\end{array}\right)$. The result is homotopic to the cone structure. Note this construction gives the identity over $U_{1}^{-5 \epsilon}$.

5.1.4 Morphism to trivial complexes. This step is an elaboration of the fact that $\left(\begin{array}{ll}1 & 0 \\ c & 1\end{array}\right)$ is a chain isomorphism of complexes

$$
\left(C \oplus S C,\left(\begin{array}{cc}
c & 1 \\
0 & -c
\end{array}\right)\right) \rightarrow\left(C \oplus S C,\left(\begin{array}{ll}
0 & 1 \\
0 & 0
\end{array}\right)\right) .
$$

This is also used in the trivialization of cancellable complexes in 3.3.3.

Conjugating the output of the first step, $(* *)$, by $\left(\begin{array}{ll}1 & 0 \\ c & 1\end{array}\right)$ gives

$$
\left(C \oplus S C,\left(\begin{array}{cc}
0 & 1 \\
c^{2} & 0
\end{array}\right),\left(\begin{array}{cc}
\xi & 0 \\
c \xi+\xi c-\xi
\end{array}\right),\left(\begin{array}{cc}
c_{i, j} & 0 \\
c c_{i, j}-c_{i, j} & c_{i, j}
\end{array}\right)\right)
$$

This is homotopic to

$$
(* * *) \quad\left(C \oplus S C,\left(\begin{array}{ll}
0 & 1 \\
0 & 0
\end{array}\right),\left(\begin{array}{cc}
0 & 0 \\
1 & 0
\end{array}\right),\left(\begin{array}{cc}
d_{i, j} & 0 \\
0 & d_{i, j}
\end{array}\right)\right)
$$


where $d_{i, j}$ is the diagonal part of $c_{i, j}$. To see this first note the chain and contraction identities and $c^{2} \sim 0$ give geometric homotopies to

$$
\left(C \oplus S C,\left(\begin{array}{ll}
0 & 1 \\
0 & 0
\end{array}\right),\left(\begin{array}{cc}
\xi & 0 \\
1 & -\xi
\end{array}\right),\left(\begin{array}{cc}
c_{i, j} & 0 \\
0 & c_{i, j}
\end{array}\right)\right) .
$$

The degree 2 homomorphism $\left(\begin{array}{ll}0 & 0 \\ \xi & 0\end{array}\right)$ provides a homotopy from this contraction to $\left(\begin{array}{ll}0 & 0 \\ 1 & 0\end{array}\right)$. Finally if the diagonal-plus-increasing decomposition of $c_{i, j}$ is $d_{i, j}+u_{i, j}$ then $\left(\begin{array}{cc}0 & 0 \\ -u_{i, j} & 0\end{array}\right)$ gives a chain homotopy from the chain map to $\left(\begin{array}{cc}d_{i, j} & 0 \\ 0 & d_{i, j}\end{array}\right)$.

This makes sense because the diagonal-plus-increasing decomposition is determined by the given data, and in particular does not depend on a particular partial order.

As in the previous step this defines a $K_{1}$ morphism of $K_{1}$ simplices. Again appropriate partial orders are obtained by shuffling image partial orders and making points comparable only if they are within $5 \epsilon$, but this time $S C$ comes after $C$ in each sub-piece to reflect the fact that $\left(\begin{array}{ll}1 & 0 \\ c & 1\end{array}\right)$ is lower triangular.

This morphism is factored in the same way as in the previous step. Let $U_{2}$ be a subset of $X$ and let $\rho$ denote projection to $U_{2}$. Factor the morphism by

$$
\left(\begin{array}{ll}
1 & 0 \\
c & 1
\end{array}\right)=\left(\begin{array}{cc}
1 & 0 \\
c(1-\rho) & 1
\end{array}\right)\left(\begin{array}{cc}
1 & 0 \\
c \rho & 1
\end{array}\right) \text {. }
$$

Write the homotopies of the contraction and chain maps as

$$
\begin{aligned}
\left(\begin{array}{ll}
0 & 0 \\
\xi & 0
\end{array}\right) & =\left(\begin{array}{cc}
0 & 0 \\
\xi(1-\rho) & 0
\end{array}\right)+\left(\begin{array}{cc}
0 & 0 \\
\xi \rho & 0
\end{array}\right) \\
\left(\begin{array}{cc}
0 & 0 \\
-u_{i, j} & 0
\end{array}\right) & =\left(\begin{array}{cc}
0 & 0 \\
-u_{i, j}(1-\rho) & 0
\end{array}\right)+\left(\begin{array}{cc}
0 & 0 \\
-u_{i, j} \rho & 0
\end{array}\right)
\end{aligned}
$$

Define a $K_{1}$ morphism from the cone structure by conjugating by the right factor, changing the contraction by the right summand, and applying homomorphism homotopies over $U_{2}^{-3 \epsilon}$. The image of this morphism has the cone structure over $X-U_{2}^{3 \epsilon}$ and the trivial structure over $U_{2}^{3 \epsilon}$.

Finally, again as in the previous step, the remainder of the morphism and homotopy give the second factor in the localization.

5.1.5 Factoring trivial complexes. The contraction of a trivial complex (i.e. of the form $(* * *))$ clearly cancels the whole complex, so the inclusion of the 0 
complex is a morphism. The final step is to factor this using the fact that trivial complexes have lots of subcomplexes whose complements cancel.

A single complex of the form $\left(C \oplus S C,\left(\begin{array}{ll}0 & 1 \\ 0 & 0\end{array}\right),\left(\begin{array}{ll}0 & 0 \\ 1 & 0\end{array}\right)\right)$ is a large sum of 2dimensional subcompexes each located over a point. Thus if $U_{3}$ is a subset of $X$ the restriction to $U_{3}$ is a subcomplex of the same form and the contraction cancels the complement so the inclusion into the whole complex is a morphism. A complication arises with simplices of complexes. The chain maps in the form $(* * *)$ are diagonal so they take one 2-dimensional summand to another, but they may have nonzero radius. Thus the image of something in $U_{3}$ may not lie in $U_{3}$. Restricting both the range and domain to $U_{3}$ may give a chain map that is not injective, so cannot be a $K_{1}$ morphism. We deal with this by subdividing: restrict both the range and domain of a chain map, but introduce a new vertex between them. The new vertex will be the subcomplex of the range obtained by adding the restriction of the range and the image of the restriction of the domain. Both vertex restrictions restrictions inject into this.

The first derived subdivision of a simplicial complex is the complex with $n$ simplices monotone sequences of length $n+1$ of subsimpices of a simplex of the original. Faces are defined by omission, and degeneracies by duplication of an element in the sequence.

Regard an $n$-simplex $\left(C, c, \xi, c_{i, j}\right)$ of $K_{1}(\epsilon)$ as the image of a map $\Delta^{n} \rightarrow K_{1}(\epsilon)$. We describe how to get a map on the first derived subdivision. A vertex of the subdivision is a subsimplex $\tau$ (monotone sequence of length 1 ). Take this to $\left(C_{i}, c_{i}, \xi_{i}\right)$, where $i$ is the largest vertex in $\tau$. Edges should go to chain maps. If $(\sigma, \tau)$ is a monotone sequence with largest vertices $i, j$ then $i \leq j$. Define the chain map $c_{\sigma, \tau}$ to be $c_{i, j}$, where we understand this to be the identity if $i=j$.

We now define the restriction of the subdivision of a "trivial" simplex. Take a vertex $\tau$ to the sum of the images in its largest vertex, of the restrictions to $U_{3}$ of all vertex complexes. Edges go to the evident restrictions and inclusions. Since all the complexes are trivial, the contractions cancel complements of all images, and this defines a simplicial map of $\left(\Delta^{n}\right)^{(1)} \rightarrow K_{1}$. Further, inclusions define a $K_{1}$ morphism of this to the unrestricted subdivision. This is our factorization of the inclusion of 0 into the original trivial simplex. The inclusion is the identity over $U_{3}$ and 0 outside $U_{3}^{\gamma}$, where $\gamma$ is the radius of the trivial simplex. The trivial 
simplices produced by earlier constructions have radius $<4 \epsilon$.

5.1.6 Proof of Proposition 5.1.1. Denote the morphisms described above by

$$
(*) \stackrel{F}{\longrightarrow}(* *) \stackrel{G}{\longrightarrow}(* * *) \stackrel{H}{\longleftarrow} 0 .
$$

Let $Y$ be the subset specified in the statement of the proposition, and let $U_{1}=$ $Y^{5 \epsilon}$. Then the construction of 5.1.3 factors $F$ as $F_{2} F_{1}$ with $F_{1}$ constant on $Y$ and $F_{2}$ constant over $X-Y^{10 \epsilon}$. Next let $U_{2}=Y^{13 \epsilon}$. Then the construction of 5.1.4 factors $G$ as $G_{2} G_{1}$ with $G_{1}$ constant on $Y^{10 \epsilon}$ and $G_{2}$ constant on $X-Y^{16 \epsilon}$. Finally let $U_{3}=Y^{16 \epsilon}$, then 5.1.5 factors $H$ as $H_{1} H_{2}$ with $H_{1}$ constant over $Y^{16 \epsilon}$ and zero outside $Y^{20 \epsilon}$.

Next we observe that two morphisms commute if one is constant where the other is nontrivial. Thus we can rearrange the sequence of morphisms to

$$
(*) \stackrel{F_{1}}{\longrightarrow} \stackrel{G_{1}}{\longrightarrow} \underset{H_{1}}{\longleftarrow} T \stackrel{F_{2}}{\longrightarrow} \stackrel{G_{2}}{\longrightarrow} \underset{H_{2}}{\longleftarrow} 0
$$

where $T_{0}$ is the result of the first three operations.

All of these morphisms have size $<7 \epsilon$ (the limiting factor being the bound on the partial orders in the first step). The intermediate functions therefore define maps into $K_{1}(7 \epsilon)$. Subdividing the morphisms gives homotopies $H_{1}, H_{2}$ as required for the Proposition. Note that the difference between the composition (in the concatenation sense) of these homotopies and the standard nullhomotopy comes from rearranging commuting $K_{1}$ morphisms. "Subdividing" the resulting commutative diagrams of morphisms gives the canonical homotopy $G$ between these two homotopies.

The other assertions in the Proposition are supposed to be easily seen from the form of the construction.

5.2 Skew inverses. In this section we use a $K_{1}$-morphism $f: B \rightarrow A$ to give a cancellation of $A \oplus S B$. The standard cancellation of inverses corresponds to the identity map $A=A$. As in the standard case this cancellation can be localized.

5.2.1 Proposition. Suppose $X, p, R$ as usual, $X \supset Y$, and $\epsilon$ is fixed. Suppose $M$ is a simplicial set, $A, B: M \rightarrow K_{1}^{l f}(X ; p, R, \epsilon)$ are simplicial and $f: B \rightarrow A$ is an $\epsilon K_{1}$ morphism (see 3.4.3). Then there are

(1) a canonical map $T: M^{(1)} \rightarrow K_{1}^{l f}(X ; p, R, 9 \epsilon)$, where the superscript (1) indicates the first derived subdivision; 
(2) canonical homotopies $H_{1}: A \oplus S B \sim T$ and $H_{2}: T \sim 0$; and

(3) a canonical homotopy $G: H_{1} H_{2} \sim H$, where $H$ is the standard cancellation (i.e. $\mathrm{H}_{2}$ for the case $Y$ empty).

These satisfy:

(1) restricted to $Y, H_{1}$ is constant equal to the identity, $H_{2}=H$, and $G$ is constant;

(2) restricted to $X-Y^{30 \epsilon}, H_{1}=H, H_{2}$ is constant equal to 0 , and $G$ is constant;

(3) $T, H_{i}$ and $G$ are natural with respect to simplicial maps of $M$ and restriction to $U, U \cap Y$, provided $Y^{60 \epsilon}-Y^{-40 \epsilon} \subset U$; and

(4) if on a subcomplex $W \subset M, A, B, f$ have radius $<\delta$, then $T, H_{i}, G$ have radius $<9 \delta$.

As in 5.1 a generous margin is used in (3) to avoid conflicts with details of the definition. Conclusion (4) can be thought of as a naturality with respect to scale. The original $\epsilon$ is used to choose subsets used in the construction, but otherwise does not enter, so radius of the output depends only on radius of the input. In fact the $\delta$ in (4) does not even have to be measured with the same metric, so (4) gives a naturality with respect to metric.

Again we shorten the notation for $K$ space to $K_{1}(\epsilon)$, and restrict attention to a single simplex of $M$ because the construction is canonical. The image of the simplex under $A$ is denoted $\left(A_{i}, a_{i}, \alpha_{i}, a_{i, j}\right)$ as usual, and simlarly for $B$. We begin by describing the full construction $A \oplus S B \sim 0$, then show how to localize it. Finally most of the construction (including estimates) follows the standard case, and we focus on new features.

5.2.2 Improvement of the image of $f$. Since $f_{i}: B_{i} \rightarrow A_{i}$ is a $K_{1}$ morphism the contractions in $A$ cancel the complement of the image. According to 3.3.4 there is a canonical triangular endomorphism $h_{i}$ of the graded module $A_{i}$ that is the identity on the image of $f$ and conjugating by $h_{i}$ changes the boundary maps so the result decomposes (as a chain complex) as the sum of the image of $f$ and its based complement. Moreover the contractions can be changed by homotopy, without disturbing the cancellation properties, to also decompose as sums.

After this modification the chain maps can be improved too. Decomposing $A_{i}$ and $A_{j}$ as image of $f$ plus based complement, the fact that $a_{i, j}$ carries the 
image of $f_{i}$ into the image of $f_{j}$ means it has the form $\left(\begin{array}{ll}x & y \\ 0 & z\end{array}\right)$. Since the boundary homomorphisms in the two complexes are diagonal it follows that $\left(\begin{array}{ll}x & 0 \\ 0 & z\end{array}\right)$ (i.e. omit the upper right term) is also a chain map. The order hypotheses on cancellations implies the difference is increasing with respect to any admissible partial order. Finally recall that diagrams of chain maps in $K_{1}$ simplices are only required to commute up to increasing homomorphisms. Putting these together shows that omitting the off-diagonal terms from the $a_{i, j}$ gives another $K_{1}$ simplex, and the identity maps on the $A_{i}$ from the original simplex to this one is a $K_{1}$ morphism.

The first step in the main construction is the $K_{1}$ morphism described above, from $A$ to the modification in which the image of $B$ splits. We proceed assuming this splitting condition.

Define $p_{i}: A_{i} \rightarrow B_{i}$ to be the based projection of $A_{i}$ to the image of $f$, composed with the inverse of $f$. Thus $p f$ is the identity of $B$, and $f p$ is the projection of $A$ to the image of $f$. After the modifications in the first step $p$ is a chain map, $f p \alpha=\alpha f p$, and $f p a_{i, j}=a_{i, j} f p$, where we have omitted evident subscripts on $f$ and $p$.

5.2.3 Morphism to cones. The sum $A \oplus S B$ has boundary maps, contractions and chain maps

$$
\left(A \oplus S B,\left(\begin{array}{cc}
a & 0 \\
0 & -b
\end{array}\right),\left(\begin{array}{cc}
\alpha & 0 \\
0 & -\beta
\end{array}\right),\left(\begin{array}{cc}
a_{i, j} & 0 \\
0 & b_{i, j}
\end{array}\right)\right) .
$$

The graded endomorphism $\left(\begin{array}{cc}1 & -f \beta \\ 0 & 1\end{array}\right)$ defines a chain map to the structure conjugated by the endomorphism, namely

$$
\left(A \oplus S B,\left(\begin{array}{cc}
a & a f \beta+f \beta b \\
0 & -b
\end{array}\right),\left(\begin{array}{cc}
\alpha & \alpha f \beta+f \beta^{2} \\
0 & -\beta
\end{array}\right),\left(\begin{array}{cc}
a_{i, j} & a_{i, j} f \beta+f \beta b_{i, j} \\
0 & b_{i, j}
\end{array}\right)\right) .
$$

Use the identity af $\beta+f \beta b \sim f$ in the boundary homomorphism. The degree-2 map

$$
\left(\begin{array}{cc}
f(\beta-p \alpha f) \beta p & \alpha f \beta^{2} \\
\beta p & \beta^{2}
\end{array}\right)
$$

gives a homotopy from the given contraction to $\left(\begin{array}{cc}\alpha(1-f p) & 0 \\ p & 0\end{array}\right)$. This takes us to

$$
\left(A \oplus S B,\left(\begin{array}{cc}
a & f \\
0 & -b
\end{array}\right),\left(\begin{array}{cc}
\alpha(1-f p) & 0 \\
p & 0
\end{array}\right),\left(\begin{array}{cc}
a_{i, j} & a_{i, j} f \beta+f \beta b_{i, j} \\
0 & b_{i, j}
\end{array}\right)\right) .
$$

At this point the contraction already gives a cancellation of the entire complex. We elaborate on this to make it easier to localize. 
5.2.4 Morphism to trivial complexes. Apply the endomorphism $\left(\begin{array}{cc}1 & 0 \\ b p & 1\end{array}\right)$ to get a morphism to a new simplex,

$$
(* * *) \quad\left(A \oplus S B,\left(\begin{array}{cc}
a(1-f p) & f \\
0 & 0
\end{array}\right),\left(\begin{array}{cc}
\alpha(1-f p) & 0 \\
p & 0
\end{array}\right),\left(\begin{array}{c}
* * \\
0 *
\end{array}\right)\right) .
$$

The triviality of the lower-left entry in the chain map is a consequence of the first step, where it was arranged that $a_{i, j} f=f p a_{i, j} f$. We will not need to track the other entries explicitly.

The simplex $(* * *)$ is based isomorphic to one of the form

$$
\left(D \oplus S D,\left(\begin{array}{ll}
0 & d \\
0 & 0
\end{array}\right),\left(\begin{array}{cc}
0 & 0 \\
d^{-1} & 0
\end{array}\right),\left(\begin{array}{l}
* 0 \\
0
\end{array}\right)\right) .
$$

where $d$ is a triangular endomorphism of the based graded module $D$. The $1-f p$ summand of $A$ has this structure by the first step. The $f p$ summand of $A$, plus $S B$, has this structure after the last step. Combining these gives the structure for the whole complex.

We simplify this further. Conjugate by $\left(\begin{array}{ll}d & 0 \\ 0 & 1\end{array}\right)$ to get a morphism to a complex of the form

$$
\left(D \oplus S D,\left(\begin{array}{ll}
0 & 1 \\
0 & 0
\end{array}\right),\left(\begin{array}{ll}
0 & 0 \\
1 & 0
\end{array}\right),\left(\begin{array}{cc}
d_{i, j} & 0 \\
0 & d_{i, j}
\end{array}\right)\right) .
$$

The final modification is to replace the $d_{i, j}$ terms in the chain maps by their diagonal maps. This is still a simplex, and the conjugation map from $(4 *)$ to this diagonalized version is still a $K_{1}$ morphism because morphisms are only required to commute modulo increasing homomorphisms.

Finally we complete the process $A \oplus S B \sim 0$ using the $K_{1}$ morphism from 0 to the complex $(5 *)$.

5.2.5 Localization. As explained in 5.1.6 it is sufficient to show how to factor the morphisms. Morphisms of the form $\left(\begin{array}{ll}1 & * \\ 0 & 1\end{array}\right)$ or alternatively of the form $1+u$ with $u^{2}=0$, can be factored over $Y$ simply by decomposing $u$ as a sum of a piece 0 over $Y$ and one 0 over $X-Y$ (see 5.1.3). Similarly modifications of contractions by homotopy can be factored by decomposing the homotopy as a sum.

The end of the argument is also easy to localize. The final complex is $(5 *)$, after adjustment to have diagonal chain maps. This decomposes as a huge sum of 2-dimensional complexes, and the cancellation is localized after subdivision as 
in 5.1.5. This explains how to localize all the steps except the morphism from $(* * *)$ to $(4 *)$ defined by $\left(\begin{array}{ll}d & 0 \\ 0 & 1\end{array}\right)$. This step does not arise in the standard-inverse cancellation because in that case $d=1$. In general we do this by factoring $d$ as $d_{2} d_{1}$ with $d_{1}=1$ over $Y$ and $d_{2}=1$ over $X-Y^{4 \epsilon}$. It is sufficient to do this when $\operatorname{diag}(d)=1$, so $d=1+u$ with $u$ increasing. Set $d_{1}=1+u \mid Y$, and $d_{2}=$ $(1+u)(1+u \mid Y)^{-1}$. The inverse is $3 \epsilon$ triangular, and over $Z=X-Y^{3 \epsilon}$ the inverse of the restriction is the restriction of the inverse: $(1+u \mid Y)^{-1}\left|Z=(1+u)^{-1}\right| Z$. Thus the composition defining $d_{2}$ reduces to 1 over a slightly smaller set.

As in 5.1.6 factoring the morphisms into commuting pieces gives a homotopy of the standard (unlocalized) homotopy to the composition of two partial homotopies with the properties claimed in Proposition 5.2.1.

5.3 Localizing homotopies. A homotopy into a $K$ space is factored into two homotopies, one that changes things away from $Y$ and one that changes things near $Y$. The thing appearing halfway through (between the two homotopies) is a spliced version of the things at the beginning and end.

5.3.1 Lemma. Suppose $M$ is a simplicial complex, $A, B: M \rightarrow K_{1}^{l f}(X ; p, R, \epsilon)$ are simplicial, and $H: A \sim B$ is a homotopy. Then there are

(1) a subdivision $M^{\prime}$ and simpicial map $C: M^{\prime} \rightarrow K_{1}(9 \epsilon)$;

(2) homotopies $H_{1}: A \sim C$ whose restriction to $Y$ is constant, and $H_{2}: C \sim$ $B$ whose restriction to $X-Y^{-60 \epsilon}$ is constant; and

(3) a homotopy $H_{1} H_{2} \sim H$.

The map and homotopies constructed have the property that if $W \subset M$ is a subcomplex and the restrictions of $A, B$ and $H$ to $W$ are in $K_{1}(\delta)$ then the output map and homotopies are in $K_{1}(9 \delta)$.

This is proved in $\S \S 5.3 .2-5.3 .4$ by describing the homotopy $A \sim B$ as obtained from a sequence of $K_{1}$ morphisms, then changing them to a "zigzag" pattern to which 5.2 applies.

The final conclusion holds even if the $\delta$ size condition uses a different metric than the one used for the $\epsilon$ conditions; see the remark after 5.2. This is used to show metric independence in $\S 5.6$, though it could be avoided with more elaborate estimates in a single metric.

The final conclusion also shows that the construction passes to homotopy 
inverse limits, though it still depends on an initial choice of $\epsilon$. Explicitly, we can regard a map of $M$ into the homotopy inverse limit as a map $M \times[n, \infty) \rightarrow$ $K_{1}(1 / n)$ that for $k>n$ takes $M \times[k, \infty)$ into $K_{1}(1 / k)$. Applying the Lemma to homotopies of such maps factors them into pieces that still have size going to 0 in the $[1 / n, \infty)$ coordinate. This gives:

Corollary. Suppose $H$ is a homotopy between maps $A, B: M \rightarrow K_{1}^{l f}(X ; p, R)$, $Y \subset X$ and $\epsilon>0$. Then $H$ is homotopic to a composition of $H_{1}: A \sim C$ and $H_{2}: C \sim B$ with the restriction of $H_{1}$ to $Y$ constant, and the restriction of $H_{2}$ to $X-Y^{60 \epsilon}$ constant.

5.3.2 Zigzag homotopies. Suppose $A \leftarrow B \rightarrow C$ are $K_{1}$ morphisms. The "zigzag homotopy" from $A$ to $C$ is obtained by composing

(1) the homotopy from $A$ to $A \oplus S B \oplus C$ obtained by adding $A$ to the skew cancellation homotopy of 5.2 for $S B \oplus C$ using the second morphism, and

(2) the homotopy from $A \oplus S B \oplus C$ to $C$ obtained by adding $C$ to the skew cancellation homotopy for $A \oplus S B$.

The name comes from the picture:

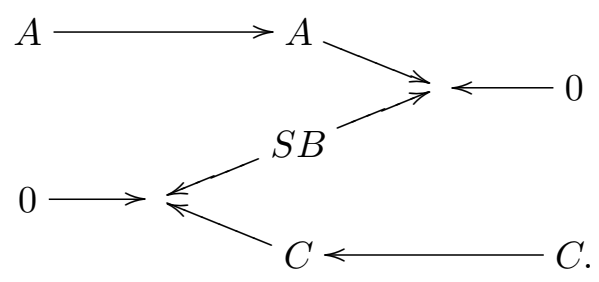

Variations on the following will be used in several places:

Lemma. Suppose $A \leftarrow B \rightarrow C$ are $K_{1}$ morphisms. Then the homotopy from $A$ to $C$ obtained by triangulating morphisms (see 3.4.4) is homotopic to the zigzag homotopy described above.

Proof. First, it is sufficient to show this for $A=B=C$. The diagram

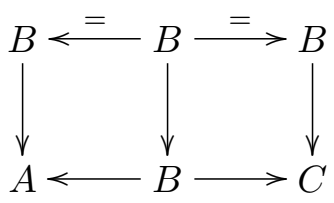


gives a homotopy between the lower homotopy $A \sim C$ and the composition of the upper (identity) homotopy $B \sim B$ with the edge homotopies $A \sim B$ and $B \sim C$. Further this diagram induces a diagram relating the morphisms used to define the zigzag homotopies. This similarly relates the two zigzag homotopies. Therefore a homotopy in the special case composes with these to give a homotopy in the general case.

Next we describe an intermediate step in the special case. Define the "eye" homotopy beginning the same way as the zigzag: by adding $B$ to the cancellation homtopy $0 \sim S B \oplus B$. But then finish by canceling the same pair rather than switching copies of $B$. The name comes from the picture:

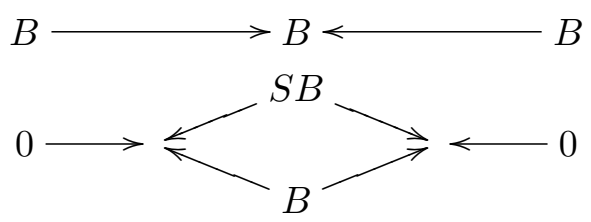

First note that this is homotopic to the triangulation homotopy (omit the eye): introducing and then removing the $S B \oplus B$ pair in exactly the same way is the composition of a homotopy $0 \sim S B \oplus B$ and its reverse, so is homotopic to the constant homotopy. on the other hand there is a basis and structure preserving isomorphism between the eye and the zigzag given by the identity on the left half and the basis bijection that interchanges the $B$ terms on the right half. This isomorphism triangulates to give a homotopy between the zigzag and the eye. Combining these observations gives a homotopy from the zigzag to the constant homotopy. This completes the Lemma.

5.3.3 The bounded case of Lemma 5.3.1. Suppose that there is a subdivision $M^{\prime}$ and a triangulation $I^{\prime}$ of $[0,1]$ so $H$ is simpicial with respect to the standard triangulation of the product $M^{\prime} \times I^{\prime}$. This is essentially a boundedness hypothesis on the number of vertices needed in the triangulation of $I$ : in general for infinite $M$ there may not be such a bound, and we explain what to do about that in 5.3.4.

When $H$ is simplicial on the product then restrictions to $M^{\prime} \times\left\{v_{i}\right\}$, where $v_{i}$ are vertices of $I^{\prime}$, gives a sequence of maps $M^{\prime} \rightarrow K_{1}(\epsilon)$. The families of chain maps associated to vertices in $M^{\prime}$ times edges in $I^{\prime}$ fit together to give morphisms 
between these maps. Triangulations of these morphisms gives a simpicial map on the same triangulation as $H$. This is not quite $H$ : in some simplices some structure maps $a_{i, j}$ are replaced by compositions $a_{k, j} a_{i, k}$, but the identity on vertices defines a morphism between this new map and $H$. They are therefore homotopic.

Denote the sequence of maps and morphisms by

$$
A_{1} \stackrel{f_{1}}{\longleftarrow} B_{1} \stackrel{g_{1}}{\longrightarrow} A_{2} \stackrel{f_{2}}{\longleftarrow} B_{2} \stackrel{g_{2}}{\longrightarrow} \cdots \longrightarrow A_{n} \stackrel{f_{n}}{\stackrel{g_{n}}{\longrightarrow}} B_{n+1}
$$

where $A_{1}$ is the subdivision of $A$ and $A_{n+1}$ is the subdivision of $B$.

The next step is a variation on the zigzag homotopy of 5.3.2. First add to $A_{1}$ the skew-inverse cancellations of pairs $S B_{i} \oplus A_{i+1}$ defined using the morphisms $g_{i}$. This gives a homotopy $A_{1} \sim A_{1} \oplus \Sigma_{i=1}^{n}\left(S B_{i} \oplus A_{i+1}\right)$. Next reassociate the sum to $\sum_{i=1}^{n-1}\left(A_{i} \oplus S B_{i}\right) \oplus A_{n}$. Canceling the indicated pairs using the morphisms $f_{i}$ gives a homotopy $\sum_{i=1}^{n}\left(A_{i} \oplus S B_{i}\right) \oplus A_{n} \sim A_{n}$. Joining these two homotopies gives a zigzag homotopy $A_{1} \sim A_{n}$. As in Lemma 5.3.2 this homotopy is homotopic to the original. It has a lot more kinks than 5.3.2, but it can be seen as a composition of a lot of simple zigzags or the proof of 5.3 .2 can easily be generalized.

This zigzag homotopy can be localized to give the statement of Lemma 5.3.1. Specifically the localized version of 5.2 splits the homotopy $A_{1} \sim A_{1} \oplus \sum_{i=1}^{n}\left(S B_{i} \oplus\right.$ $\left.A_{i+1}\right)$ into a homotopy constant over $Y$ and one constant over $X-Y^{-30 \epsilon}$. Similarly split the homotopy $\sum_{i=1}^{n}\left(A_{i} \oplus S B_{i}\right) \oplus A_{n} \sim A_{n}$ into a piece constant over $Y^{30 \epsilon}$ and one constant over $X-Y^{60 \epsilon}$. The middle pieces in the composition of these four homotopies commute (each is constant where the other is not). Interchanging them gives the desired factorization.

5.3.4 The general case. By subdivision and straightforward manipulations we can arrange $M^{\prime}$ to be a union of closed subcomplexes $N_{i}$ with

(1) $N_{i} \cap N_{j}$ is empty unless $i, j$ are equal or differ by one;

(2) $N_{i} \cap N_{i+1}$ is collared in each larger complex (e.g. like the boundary of a regular neighborhood);

(3) restricted to $N_{i} \times I, H$ is simplicial on the standard triangulation of $N$ times a triangulation $I_{i}$ of $I$, and the triangulation $I_{i+1}$ is a subdivision (i.e. obtained by adding vertices) of $I_{i}$. 
As in 5.4.2 the vertices of $I_{i}$ give maps $N_{i} \rightarrow K_{1}(\epsilon)$, and the edges give morphisms between these maps. On $N_{i} \cap N_{i+1}$ the longer sequence is obtained by inserting identity morphisms into the shorter.

We can use the bounded-length construction to produce morphisms, homotopies etc. on each $N_{i}$. These are patched together using the method of the zigzag homotopy lemma. We outline the argument.

Construct an intermediate object by modifying the $i+1$ procedure: introduce the alternating sum as before, but then reassociate differently. On the terms in the $i$ sequence associate as in the $i$ sequence. Leave the new (identity) terms alone. Now cancel the paired terms to get a sequence of morphisms.

There is a basis and structure preserving isomorphism that matches up the associations used in the original $i+1$ and modified $i$ cancellations. This extends to a commutative diagram between the two output sequences of morphisms. This gives a homotopy between the $i+1$ sequence and the intermediate one.

On the other hand the intermediate sequence is the sum of the $i$ sequence and a lot of terms obtained by introducing and then canceling in the same way, identity morphisms. On the homotopy level this corresponds to composing a homotopy with its inverse, so the result is canonically homotopic to the constant homotopy.

The conclusion is that there is a canonical homotopy between the homotopies on $N_{i} \cap N_{i+1}$ produced by the $i$ and $i+1$ constructions. Define maps on the union by using this homotopy in a collar on one side.

5.4 The homotopy axiom. The homotopy axiom for controlled $K$-theory is the assertion that that the inclusion $X \times\{0\} \subset X \times I$ induces a homotopy equivalence of $K_{1}^{l f}$ spaces. We reduce this to the more primitive statement 5.4.1, then prove that using cancellations. The proof is similar to that of 5.3, but here we are producing homotopies rather than modifying them.

First note projection $X \times I \rightarrow X$ also defines a map of $K$ spaces, and the composition $X \times\{0\} \rightarrow X \times I \rightarrow X$ is the identity. Thus projection provides a homotopy inverse if we can show the other composition induces a map homotopic to the identity. The other map is the endomorphism of $K_{1}^{l f}(X \times I ; p \times I, R)$ defined by projecting objects to $X \times\{0\}$.

The second observation is that the $K$ spaces involved here are homotopy 
inverse limits as $\epsilon \rightarrow 0$, of $\epsilon$ spaces (see 4.4). Thus it is sufficient to do an $\epsilon$ version for each $\epsilon$ and show they are compatible up to homotopy. The statement we need is:

5.4.1 Proposition. Suppose $X, p, R$ are as usual and $\epsilon>0$, then there is a standard homotopy between the relax-control inclusion

$$
K_{1}^{l f}(X \times I ; p \times I, R, \epsilon) \longrightarrow K_{1}^{l f}(X \times I ; p \times I, R, 8 \epsilon)
$$

and the map induced by projection to $X \times\{0\}$, followed by inclusion. If $\epsilon>\delta$ then there is a homotopy between the $\delta$ homotopy and the composition of $K_{1}(\delta) \rightarrow$ $K_{1}(\epsilon)$ and the $\epsilon$ homotopy.

The proof uses only the unlocalized standard-inverse version of cancellation. The constructions are canonical so it is sufficient to describe the effect on a single simplex $\left(C, c, \xi, c_{i, j}\right)$ of $K_{1}^{l f}(X \times I ; p \times I, R, \epsilon)$.

Suppose $\epsilon=1 / n$, and choose maps $p_{i}, 0 \leq i \leq n$ that push $X \times I$ toward the 0 end, so that

(1) $p_{n}$ is the identity;

(2) $p_{0}$ is the projection to $X \times\{1\}$; and

(3) the distance from $p_{i}$ to $p_{i+1}$ is less than $\epsilon$.

For instance $p_{i}(x, t)=(x, i \epsilon t)$. In these terms the object is to define a homotopy from $p_{n}(C)$ to $p_{0}(C)$.

The first step is to use 5.1 to get a $7 \epsilon$ homotopy

$$
p_{n}(C) \sim\left(\sum_{i=0}^{n-1} p_{i}(C) \oplus S p_{i}(C)\right) \oplus p_{n}(C) .
$$

Reassociate this to

$$
p_{0}(C) \oplus\left(\sum_{i=0}^{n-1} S p_{i}(C) \oplus p_{i+1}(C)\right) .
$$

Next use $d\left(p_{i}, p_{i+1}\right)<\epsilon$ to change the $p_{i}(C)$ terms to $p_{i+1}(C)$ terms. More formally we can think of this as an $\epsilon$ morphism of $K_{1}$ objects, so it subdivides to give a homotopy. The final step uses 5.1 backwards to cancel the resulting pairs $S p_{i+1}(C) \oplus p_{i+1}(C)$, leaving $p_{0}(C)$. This gives a "zigzag" homotopy in the sense of 5.3 from $p_{n}(C)$ to $p_{0}(C)$. 
Note that on $K_{1}(\delta)$ the cancellations gives $7 \delta$ homotopies, but moving $p_{i}$ to $p_{i+1}$ is still an $\epsilon$ move. The size of the final homotopy is therefore limited by the number of intermediate pieces used, not the size of the input.

Now we must relate the homotopies obtained at different levels. Suppose $k \delta=\epsilon$. The prescription for $\delta$ calls for $k$ times as many shifted copies of $C$ as for $\epsilon$, and we can think of the $\epsilon$ process as involving the copies with index a multiple of $k$. This identifies the $\delta$ homotopy as obtained by putting extra zigzags in the $\epsilon$ homotopy, and therefore homotopic by Lemma 5.3.2. We go through the argument to clarify where the size estimates enter.

As an intermediate step in the comparison we introduce the additional copies in the $\epsilon$ process as well, but then cancel them back out without rearranging them. On the homotopy level this corresponds to composing a homotopy and its inverse, so this modified process gives a homotopy homotopic to the $\epsilon$ homotopy.

The modified $\epsilon$ process starts out introducing the same $p(C) \oplus S p(C)$ pairs as the $\delta$ process, but they are grouped differently for the second cancellation. The differences separate into blocks of length $k+1$. Expicitly, in a sequence

$$
\left(p_{k i}(C) \oplus S p_{k i}(C)\right) \oplus\left(p_{k i+1}(C) \oplus S p_{k i+1}(C)\right) \cdots\left(p_{k(i+1)}(C) \oplus S p_{k(i+1)}(C)\right)
$$

the $\delta$ process reassociates linearly, while the $\epsilon$ process associates $S p_{k i}(C)$ with $p_{k(i+1)}(C)$ and leaves the terms in between as they are. Note there is a permutation of the $S p_{*}(C)$ terms that takes the associate of each $p_{j}(C)$ in one association to its associate in the other. We can think of this as giving a basis-preserving endormorphism of the sum that preserves all $K_{1}$ structure. The cancellation construction is natural with respect to basis-preserving isomorphisms, so this isomorphism extends to a commutative diagram of morphisms between the morphisms used to define the cancellations. Triangulating this commutative diagram gives a homotopy between the homotopies.

Finally since the permutation preserves the blocks of length $k+1$ and the shifted copies in such a block differ by less than $\epsilon$, it follows that the based isomorphisms and therefore the homotopies obtained from them do not increase radius more than $\epsilon$. This means the $\epsilon$ and $\delta$ homotopies are homotopic as maps into $K_{1}^{l f}(X \times I ; p \times I, R, 8 \epsilon)$. This is the coherence claimed in 5.4.1, so the proof is complete. 
5.5 The exactness axiom. The exactness axiom 6.1(3) for $K$-theory is the assertion that given a morphism in the category, i.e. a commutative diagram

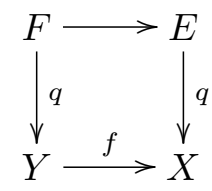

with $f$ proper, the induced sequence

$K_{1}^{l f}(Y \times\{1\} ; q, R) \rightarrow K_{1}^{l f}\left(Y \times I \cup_{f} X ; q \times I \cup p, R\right) \rightarrow K_{1}^{l f}\left(Y \times I \cup_{f} X ; q \times[0,1) \cup p, R\right)$ is a homotopy fibration. The notation $Y \times I \cup_{f} X$ indicates the mapping cylinder, with the $Y \times 0$ end identified with its image in $X$.

In 5.5.1 we describe a homotopy lifting property that implies a sequence is a homotopy fibration. Lemma 5.5.2 is a $K_{1}$ statement that follows from the homotopy localization lemma 5.3.1, and that together with the homotopy invariance proved in 5.4 shows that the lifting property is satisfied.

5.5.1 A homotopy lifting property. A sequence

$$
F \stackrel{r}{\longrightarrow} E \stackrel{p}{\longrightarrow} B
$$

with $r$ and inclusion and $p r=*$ is a homotopy fibration if it has the following property: given $M \rightarrow E$ and a homotopy $p f \sim *$ then there are

(1) a map $\hat{f}: M \rightarrow F$;

(2) a homotopy $\hat{h}: f \sim r \hat{f}$; and

(3) a homotopy between $h$ and $p \hat{h}$

such that if $W \subset M$ has $f(W) \subset r(F)$ and $h \mid W=*$ then $\hat{f}|W=f| W$ and the homotopy of (3) is constant on $W$.

This is not a general definition, but it implies the general definition and fits the need. Convert $p$ into a Hurewicz fibration (e.g. using the path space construction), then $p r=*$ defines a map from $F$ to the fiber. The lifting property applied with $M$ the homotopy fiber gives a map in the other direction. The special properties of the lift show it is a homotopy equivalence (in fact a deformation retraction).

The next statement is an approximation to this lifting property for the spaces of the axiom. 
5.5.2 Lemma. Suppose $f: M \rightarrow K_{1}^{l f}\left(Y \times I \cup_{f} X ; q \times I \cup p, R\right)$ is a map, and $h: p f \sim 0$ is a homotopy, where $p$ is the restriction to $K_{1}^{l f}\left(Y \times I \cup_{f} X ; q \times\right.$ $[0,1) \cup p, R)$. Then $h$ is homotopic by a homotopy constant over the complement of $Y \times[1 / 4,3 / 4]$ to a composition $\bar{h}_{1} \bar{h}_{2}$ with $\bar{h}_{1}$ constant over $Y \times[3 / 4,1]$ and $\bar{h}_{2}$ constant over $Y \times[0,1 / 4] \cup_{f} X$.

This follows from the homotopy localization lemma, more specifically from the Corollary of 5.3.1, where the $Y$ in the Corollary is replaced by $Y \times[3 / 4,1]$ and $\epsilon$ is chosen so $60 \epsilon<1 / 2$.

This Lemma implies the lifting property as follows: let $\bar{f}$ be the result of the homotopy $\bar{h}_{1}$. Since $\bar{h}_{2}$ is constant over $Y \times[0,1 / 4] \cup_{f} X$ and the end result is $0, \bar{f}$ is 0 over this space. Thus composing $\bar{f}$ with the projection to $Y \times\{1\}$ gives a map $\hat{f}: M \rightarrow K_{1}^{l f}(Y \times\{1\} ; q, R)$. The homotopy axiom gives a homotopy between the projection and the inclusion of $Y \times[0,1 / 4] \cup_{f} X$. Composing this homotopy with $\bar{f}$ gives a homotopy $\hat{f} \sim \bar{f}$. Composing this with the homotopy $\bar{h}_{1}$ gives a homotopy $\hat{h}: f \sim \hat{f}$. Finally the homotopy $\bar{h}_{2}$ gives the homotopy-of-homotopies (3) in the lifting property. Notice that none of this changes something that starts out over $Y \times\{1\}$, so $f, h$ are unchanged on the subset $W \subset M$ that maps into $K_{1}^{l f}(Y \times\{1\} ; q, R)$. This verifies the final condition in the lifting property, and completes the proof that the sequence is a homotopy fibration.

5.6 Metric invariance. The last ingredient needed for the axioms of $\S 6$ is independence of the metric. General principles (4.4.2) only give functorality with respect to uniformly continuous maps of metric spaces, while the axioms require functoriality with respect to all proper maps. We show:

5.6.1 Proposition. Suppose $X$ is locally compact and $d_{1} \leq d_{2}$ are metrics on $X$. Then the map

$$
K_{1}^{l f}\left(\left(X, d_{2}\right) ; p, R\right) \rightarrow K_{1}^{l f}\left(\left(X, d_{1}\right) ; p, R\right)
$$

induced by the identity map is a homotopy equivalence.

Note the condition $d_{1} \leq d_{2}$ implies the identity is uniformly continuous, so it does induce a map (in fact an inclusion) of $K$ spaces. Also this is not a version of the stability theorem 2.3.1: here we get different control by rearranging the data at hand; there we get better control. 
This relates to general functoriality as follows: suppose there is a morphism (including reference spaces) over a proper map $f: X \rightarrow Y$ of metric spaces. Define a new metric on $X$ by $d_{2}(x, y)=\max \left(d_{X}(x, y), d_{Y}(f(x), f(y))\right)$. The maps

$$
\left(X, d_{X}\right) \stackrel{\text { id }}{\longleftarrow}\left(X, d_{2}\right) \stackrel{f}{\longrightarrow}\left(Y, d_{Y}\right)
$$

are then uniformly continuous and so induce maps on controlled $K$. According to the Proposition the first map induces a homotopy equivalence, and the "metric independent" induced map is supposed to be the composition of the second with a homotopy inverse. But since homotopy inverses are not well-defined these "induced map" are not well-defined, let alone functorial.

The technical fix for this is to change the definition. Define " $\epsilon$ control" with respect to functions $\epsilon: X \rightarrow(0, \infty)$ (see 2.1.3 and [Quinn 2]), and define $K_{1}^{l f}$ to be the homotopy inverse limit over all such functions, not just the constants. This inverse system is much larger, so for instance the path model for the homotopy inverse limit does not apply. However the result is metric-independent and fully functorial.

In this context a version of Proposition 5.6.1 asserts that the map of the constant-control version into the function-control version is a homotopy equivalence. This means the only need for the function-control version is to provide full functoriality: all of the real mathematics (axiom verifications, etc.) can be done in the constant-control version. Accordingly we have focused on constant control in this paper.

The following is a single-metric statement in which non-constant control is obtained from constant control. Constant control with respect to a second metric follows easily from non-constant control with respect to the first. To keep the notation consistent with 5.6.1 the single metric is denoted " $d_{2}$ ".

5.6.2 Lemma. Suppose $Y_{i} i \geq 1$ are increasing compact subspaces of a metric space $X$ as above, $\delta_{j}$ is a positive decreasing sequence, and $Y_{i}^{60 \delta_{i}} \subset Y_{i+1}$. Suppose $f: M \times[m, \infty) \rightarrow K_{1}^{l f}\left(X ; p, R, \delta_{1}\right)$ is a map so that the restriction to $M \times[k, \infty)$ maps into $K_{1}(1 / k)$. Then there is a homotopy $H: f \sim \hat{f}$ in $K_{1}\left(9 \delta_{1}\right)$ so that

(1) $H$ maps $M \times[k, \infty) \times I$ into $K_{1}(9 / k)$;

(2) images of $\hat{f}(M \times[k, \infty))$ have radius $<9 \delta_{i+k}$ over $X-Y_{i}$; and 
(3) if $d_{1}$ is another metric on $X$ and $W \subset M$ has images of $f(W \times[k, \infty))$ of $d_{1}$ radius $<\rho$ then images of $H(W \times[k, \infty) \times I)$ have $d_{1}$ radius $<9 \rho$.

5.6.3 Proof of 5.6.1 from Lemma 5.6.2. Suppose $Y_{i} \subset X$ an increasing sequence of compact subspaces with $X=\cup Y_{i}$. Then compactness implies that there are numbers $\delta_{i, n}>0$ so that for any $n$ and $x, y \in X$, if $x, y \notin Y_{i}$ implies $d_{2}(x, y)<\delta_{i, n}($ all $i)$ then $d_{1}(x, y)<\frac{1}{n}$. Take $\delta_{k}=\min \left\{\delta_{i, j} \mid i+j=k\right\} / 9$, and reduce further if necessary so the conditions of the lemma are also satisfied: it is monotone decreasing and $Y_{i}^{60 \delta_{i}} \subset Y_{i+1}$.

Use the path model for the homotopy inverse limit to identify $K_{1}^{l f}\left(\left(X, d_{2}\right) ; p, R\right)$ as a space of maps $[1, \infty) \rightarrow K_{1}^{l f}\left(\left(X, d_{2}\right) ; p, R, \epsilon\right)$ so that the radius of the image of $[k, \infty)$ goes to 0 as $k$ goes to $\infty$. Let $M$ be the subset that satisfies the estimate of the lemma: radius $<1 / k$ on $M \times[k, \infty)$, and similarly $M_{9}$ is the subset with radius $<9 / k$ on the same subset. It is easily seen that the inclusion of $M$ and $M_{9}$ into the whole path space are homotopy equivalences. Let $W$ be the subspace of $M$ on which the $d_{1}$ radii also go to 0 , and $W_{9}$ the corresponding subset of $M_{9}$. Then $W, W_{9}$ are homotopy equivalent to $K_{1}^{l f}\left(\left(X, d_{1}\right) ; p, R\right)$. The objective is to show that the inclusion $W \subset M$ is a homotopy equivalence.

Let $H$ be a homotopy as provided by the lemma, applied to the "evaluation map" $M \times[m, \infty) \rightarrow K_{1}^{l f}\left(X ; p, R, \delta_{1}\right)$. Conclusion (1) of the lemma shows this defines a homotopy in $M_{9}$ beginning with the inclusion $M \subset M_{9}$ and ending with a map $\hat{f}$. Conclusion (2) and the choice of $Y_{i}, \delta_{i, j}$ shows that $\hat{f}$ has image lying in $W$. Finally conclusion (3) shows that points in $W$ stay inside $W_{9}$ during the homotopy. These together with the facts that $M \subset M_{9}$ and $W \subset W_{9}$ are homotopy equivalences show that $\hat{f}$ gives a homotopy inverse for the inclusion.

5.6.4 Proof of Lemma 5.6.2. The starting data is

$$
f: M \times[m, \infty) \rightarrow K_{1}^{l f}\left(X ; p, R, \delta_{1}\right)
$$

so that the restriction to $M \times[k, \infty)$ maps into $K_{1}(1 / k)$. Choose $n_{i}$ so that $1 / n_{i}<\delta_{i}$. Denote by $f_{i}$ the restriction of $f$ to $M \times\{i\}$.

The objective is to define a homotopy $H: M \times[m, \infty) \times[0,1] \rightarrow K_{1}\left(9 \delta_{1}\right)$. We first describe $H$ on slices $M \times\{k\} \times[0,1]$, then describe how to fill in between these slices. 
Suppose $H$ is defined on $M \times\{k\} \times\left[0,1-\frac{1}{r}\right]$, and on $M \times\{k\} \times\left\{1-\frac{1}{r}\right\}$ the restriction over $X-Y_{r}$ is equal to $f_{n_{k+r}}$. $f$ on $M \times\left[n_{k+r}, n_{k+r+1}\right]$ gives a homotopy of radius $<\frac{1}{n_{k+r}}<\delta_{r}$ from $f_{n_{k+r}}$ to $f_{n_{k+r+1}}$. Use 5.3 to factor this into a homotopy $G_{1}$ constant over $Y_{r}$ and $G_{2}$ constant over $X-Y_{r}^{60 \delta_{r}}$. $G_{1}$ also extends by the constant homotopy over $Y_{r}$ to define a homotopy starting with $H \mid M \times\{k\} \times\left\{1-\frac{1}{r}\right\}$. Use this modified version of $G_{1}$ to define $H$ on $M \times\{k\} \times$ $\left[1-\frac{1}{r}, 1-\frac{1}{r+1}\right]$. Doing this for all $r$ defines $H$ on the open interval $M \times\{k\} \times[0,1)$. This extends continuously to a map on the closed interval since it is eventually constant when restricted to any compact set. Specifically the restriction to $Y_{r}$ is constant (as a function of the last coordinate) on $\left.M \times\{k\} \times\left[1-\frac{1}{r+1}, 1\right)\right]$.

This defines $H$ on slices $M \times\{k\} \times[0,1]$. We check estimates for the final map, on $M \times\{k\} \times\{1\}$. Over $Y_{r+1}-Y_{r}$ this is a "splicing" of $f_{n_{k+r}}$ and $f_{n_{k+r+1}}$, obtained as the result of the first piece of a factoring of the homotopy between them $\left(G_{1}\right.$ above). It therefore has radius bounded by 9 times the radius of the homotopy, $\frac{1}{n_{k+r}}$ in this instance. Since these estimates decrease with $r$ we see that over $X-Y_{r}$ the radius is bounded by $\frac{9}{n_{k+r}}$ as required for the lemma.

With this description of $H$ on slices it should be clear how to fill in between them. We describe it explicitly at the $r^{\text {th }}$ stage of the construction, i.e. how to define $H$ on $M \times[k, k+1] \times\left\{1-\frac{1}{r}\right\}$. Over $X-Y_{r}$ the $k$ end is $f_{n_{k+r}}$ and the $k+1$ end is $f_{n_{k+r+1}}$, and we join them with $f \mid\left[n_{k+r}, n_{k+r+1}\right]$. If $s \leq r$ then over $Y_{s}-Y_{s-1}$ the $k$ end is the result of the first factor $G_{1}$ in a localization factorization $G_{1} G_{2}$ of the homotopy $f \mid\left[n_{k+s-1}, n_{k+s}\right]$. The $k+1$ end is the result of a similar factorization $G_{1}^{\prime} G_{2}^{\prime}$ of $f \mid\left[n_{k+s}, n_{k+s+1}\right]$. We fill in between these with $G_{2} G_{1}^{\prime}$. These descriptions over $Y_{s}-Y_{s-1}$ fit together to define a homotopy over all of $X$. This defines $H$ on intervals $M \times[k, k+1] \times\left\{1-\frac{1}{r}\right\}$, and therefore on boundaries of rectangles $M \times[k, k+1] \times\left[1-\frac{1}{r}, 1-\frac{1}{r+1}\right]$. But the two paths on this boundary from the $k, 1-\frac{1}{r}$ corner to the $k+1,1-\frac{1}{r+1}$ corner are the same, so the rectangle is easily filled in.

This completes the construction of the homotopy $H$. The size estimate is verified during the proof. Other properties follow from properties of homotopy localization 5.3.

\section{Homology}

This section gives the Characterization Theorem 6.1 needed for the controlled 
assembly isomorphism theorem 2.2.1. This is a sharpened version of Theorem 8.5 of [Quinn 2], and more details are given. Some of the terms used in the statement are defined and developed in 6.2. Section 6.3 combines restriction and induced maps in a single functor structure. 6.4 derives the traditional form of excision. The spectrum structure on $J$ is defined in 6.5 , and spectral cosheaves constructed in 6.6. Homology with coefficients in these is defined in 6.7, and assembly maps constructed in 6.8. The theorem is proved in 6.9. Finally 6.11 provides an "iterated homology identity" that gives a Leray-Serre type spectral sequence for homology of the domain of a morphism.

6.1 Characterization theorem. Suppose $\mathcal{C}$ is a category of maps over locally compact spaces and proper morphisms, and suppose $J: \mathcal{C} \rightarrow$ (pointed $\mathrm{CW})$ is a functor satisfying the following axioms (see notes for explanations):

(1) (Homotopy) if $(X, p)$ is an object then the inclusion-induced map

$$
J(X \times\{1\}, p) \rightarrow J(X \times I, p \times \text { id })
$$

is a homotopy equivalence;

(2) (Restriction) if $(X, p)$ is a object and $U \subset X$ is open then there is a natural restriction map $J(X ; p) \rightarrow J(U, p \mid U)$ that takes $J(X-U ; p \mid(X-$ $U)$ ) to the basepoint;

(3) (Exactness) if $f:(Y, q) \rightarrow(X, p)$ is a morphism in $\mathcal{C}$ then

$J(Y \times\{1\}, q) \stackrel{\text { incl }}{\longrightarrow} J\left(Y \times[0,1] \cup_{f} X, q \times \operatorname{id} \cup p\right) \stackrel{\text { rest }}{\longrightarrow} J\left(Y \times[0,1) \cup_{f} X, q \times \operatorname{id} \cup p\right)$ is a homotopy fibration sequence;

(4) (Unions) if $X$ is a disjoint union of open sets $U_{\alpha}$ then the product of the restrictions

$$
J(X, p) \rightarrow \prod_{\alpha} J\left(U_{\alpha}, p \mid U_{\alpha}\right)
$$

is a homotopy equivalence.

Then $J$ has a natural (possibly non-connective) $\Omega$-spectrum structure denoted $\mathbb{J}$. If $p: E \rightarrow X$ is an object of $\mathcal{C}$ with $X$ a locally compact $A N R$ and $p$ a stratified system of fibrations then there is a spectral cosheaf $\mathbb{J}(p) \rightarrow X$ defined by applying $\mathbb{J}$ "fiberwise", and the assembly map

$$
\mathbb{H}^{l f}(X ; \mathbb{J}(p)) \rightarrow \mathbb{J}(X, p)
$$


is an equivalence of spectra.

\subsubsection{Notes.}

(1) In the application here the category $\mathcal{C}$ has objects $E \rightarrow X$ with $X$ locally compact and metric, and morphisms are commutative diagrams with maps on the locally compact spaces required to be proper.

In other applications if it is hard to get naturality on this category a smaller one might be used. What we need of $\mathcal{C}$ is that it is closed under mapping cylinders, passing to open subsets of the target space, and taking products with finite $\mathrm{CW}$ complexes, and that it contains the maps with $X$ a locally compact $\mathrm{CW}$ complex and $E$ a stratified system of fibrations over a filtration of $X$ by subcomplexes.

(2) The space $Y \times[0,1] \cup_{f} X$ in (3) is the mapping cylinder of $f$ defined by the relation $(y, 0) \sim f(y)$ on the disjoint union. The maps are obtained by including and then deleting the outer end of the mapping cylinder.

(3) In (1) note that $X \times I$ is the mapping cylinder of the identity map. Thus if (3) holds, (1) is equivalent to " $J(X \times[0,1), p)$ is contractible". However (1) as stated seems to be more basic and slightly easier to verify in the application.

(4) The union axiom (4) gives new information only in the infinite case, since finite unions follow from the other axioms. Note the map is to the product with the product topology, so in the infinite case homotopy groups are products, not sums, of homotopy of the factors.

6.2 Extended naturality. Restriction and naturality with respect to proper maps can conveniently be combined into a single structure. Let $\mathcal{C}^{+}$denote the category of maps $p: E \rightarrow X$ of pointed spaces with $X$ compact and restriction to the complement of the basepoint gives an object in $\mathcal{C}$. Denote the basepoint by " $\infty$ ", then explicitly this means $p^{-1}(X-\infty) \rightarrow(X-\infty)$ is an object in $\mathcal{C}$.

There is a functor $\mathcal{C} \rightarrow \mathcal{C}^{+}$, taking $E \rightarrow X$ to $E^{+} \rightarrow X^{+}$where $X^{+}$is the 1-point compactification and $E^{+}$is $E \cup \infty$ with neighborhoods of $\infty$ given by inverse images of complements of compact sets in $X$.

6.2.1 Lemma. the functor $J$ extends to $\mathcal{C}^{+}$

$J$ is defined on objects simply by applying the original functor to the comple- 
ment of the basepoint. For morphisms note that if $f: X \rightarrow Y$ is a basepointpreserving map of compact spaces then we get

$$
X-\infty \stackrel{\text { open subset }}{\longleftarrow} f^{-1}(Y-\infty) \stackrel{\text { proper }}{\longrightarrow} Y-\infty
$$

and similarly for reference maps over these. The restriction and induced maps give

$$
J(X ; p) \rightarrow J\left(f^{-1} Y ; p \mid f^{-1} Y\right) \rightarrow J(Y ; q)
$$

and the composition of these is the desired induced map.

6.2.2 Caution. One-point compactification does not make wild behavior of $X$ at infinity go away, but just packs it into a neighborhood of the basepoint. Consequently the basepoint must be treated with care.

For example if we begin with $X$ a locally compact CW complex with filtration by skeleta and 1-point compactify it, the result is almost never a CW complex; the inclusion of the basepoint is not a cofibration; and filtering $X^{+}$by adding the basepoint as a new stratum does not give a filtration even dominated by a CW filtration. Compactification is a useful trick in simplifying naturality, and useful in defining locally finite homology in 6.7 .3 , but does not avoid any of the real work needed to deal with noncompact spaces.

6.3 Excision. Excision relates locally finite homology of a closed subspace to homology of its complement. However this is only expected for cofibered subspaces, even for ordinary homology.

6.3.1 Cofibrations. We recall that a subspace $Y \subset X$ is a cofibration if $Y \times I \cup$ $X \times\{0\}$ is a retract of $X \times I$. Explicitly this is a map $X \times I \rightarrow Y \times I \cup X \times\{0\}$ that is the identity on the subspace. To say it gives a cofibration in a category of maps, as below, means there should be a retraction that lifts to a retraction of of reference maps. This is a more categorical version of the older term " $p$ neighborhood deformation retract" [Quinn 2].

6.3.2 Lemma. If $(Y, q) \rightarrow(X, p)$ is a cofibration in $\mathcal{C}$ then the sequence

$$
J(Y, q) \rightarrow J(X, p) \rightarrow J(X-Y, p)
$$

is a homotopy fibration. 
The first map in the sequence is induced by the inclusion, the second by restriction to the complement. The definition of cofibration provides a morphism $X \times I \rightarrow X \times\{0\} \cup Y \times I$ extending the identity. Such a map can be spread over two variables to give a homotopy of itself to the identity. Thus $X \times\{0\} \cup Y \times I$ is a deformation retract of $X \times I$ in the category.

Consider the diagram, in which reference maps are suppressed:

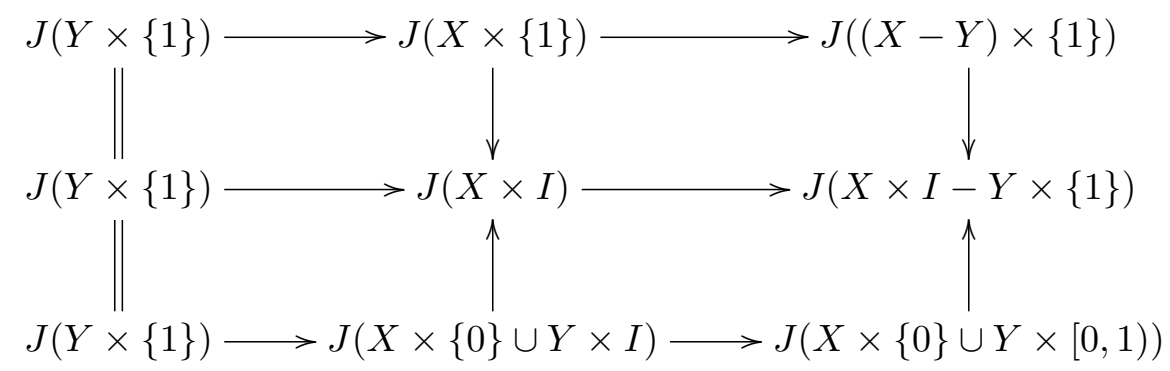

The bottom row is a homotopy fibration according to the exactness axiom. The top row is the excision sequence. The lemma will therefore follow if we show the vertical maps are equivalences.

The left verticals are identities, so equivalences. The upper middle is an equivalence by the homotopy axiom. The lower middle is an equivalence because it is induced by an inclusion that is a deformation retract in the category (the cofibration hypothesis). Showing the right verticals are equivalences is simplified by using extended functoriality. Taking 1-point compactifications and dividing out $Y \times\{1\}$ shows the lower right to induced by the inclusion

$$
\left(X^{+} \times\{0\} \cup Y^{+} \times I\right) /(\infty \times I \cup Y \times\{1\}) \subset X^{+} \times I /(\infty \times I \cup Y \times\{1\}) .
$$

But the deformation retraction of $X \times I$ passes to the quotient to give a deformation retraction for this inclusion, so it induces an equivalence. Finally for the upper right, the standard deformation retraction of $X \times I$ to $X \times\{1\}$ gives a deformation retraction of $X^{+} \times I /(\infty \times I \cup Y \times\{1\})$ to $X^{+} \times\{1\} /(\infty \times I \cup Y \times\{1\})$. Therefore this also is an equivalence.

6.4 Spectrum structure. According to excision (6.3.2) the sequence

$$
J(X \times\{0\}, p) \rightarrow J(X \times[0,1), p) \rightarrow J(X \times(0,1))
$$


is a homotopy fibration sequence. But it follows from the homotopy axiom that the middle space is contractible. The homotopy fiber of the point map is the loop space, so we get a homotopy equivalence $\Omega(J(X \times R)) \rightarrow J(X)$. This equivalence is natural up to essentially canonical homotopy.

Recall that an $\Omega$-spectrum is a sequence of spaces $J_{n}$ with homotopy equivalences $\Omega J_{n} \rightarrow J_{n+1}$. We therefore make $J(X, p)$ into a spectrum with $J_{n}(X, p)=$ $J\left(X \times R^{n}, p\right)$ if $n \geq 0$, and for negative $n J_{n}(X, p)=\Omega^{-n} J(X, p)$. Denote this spectrum by $\mathbb{J}(X, p)$.

Lemma. This construction extends $J$ to a functor $\mathbb{J}: \mathcal{C}^{+} \rightarrow \Omega$ spectra.

There are minor homotopy issues with the structure maps not quite being canonical. Fixes for this do not illuminate anything so are not included.

6.5 Homotopy stratified maps. These are slightly more general than "stratified systems of fibrations". They occur frequently in applications and come up in the next section.

6.5.1 Definition. A map $p: E \rightarrow X$ is homotopy stratified if there is a closed filtration $X^{0} \subset \cdots \subset X^{n} \subset \ldots$ so that

(1) the restriction to each stratum, $p^{-1}\left(X^{j}-X^{j-1}\right) \rightarrow\left(X^{j}-X^{j-1}\right)$, is an approximate fibration, and

(2) the inclusions $X^{j} \rightarrow X$ are approximate cofibrations in the category of maps.

An approximate fibration is a map with an approximate lifting property, see [Quinn 1]. An approximate cofibration is defined by reversing the maps. For the purposes here the following characterization could be used as a definition of approximate fibration:

6.5.2 Lemma. A map is homotopy stratified if and only if the stratified homotopy link in the mapping cylinder is a stratified system of fibrations.

Let $\operatorname{cyl}(p)$ denote the mapping cylinder of $p: E \rightarrow F$. The stratified homotopy link is the space of maps $[0,1] \rightarrow \operatorname{cyl}(p)$ so that 0 maps to $X,(0,1]$ maps into the complement of $X$ in the inverse image of the stratum containing the image of 0 . The proof in the unstratified case is given in [Quinn 5, §2.7], and the general 
case follows directly from this. For stratified homotopy links see also [Hughes, 1] and [Quinn 8].

Evaluation gives a canonical "approximate morphism" holink $(\operatorname{cyl}(p)) \times(0,1] \rightarrow$ $E$ that is in the approximate sense a fiber homotopy equivalence. In these terms an approximate fibration is canonically approximately fiber homotopic to a genuine fibration, (the homotopy link) and we systematically use this to avoid working on the homotopy level with approximate things.

6.6 Functorial approximation. When working with cohomology one constructs a sheaf easily with no hypotheses: take an open set $U$ to $J(U)$, and inclusions to restriction morphisms. The cosheaf construction involves applying $J$ to point inverses rather than open sets. Locating appropriate "point inverses" seems to require the stratification hypotheses and some work. In this section we show that the coefficient maps we work with can be approximated by realizations of functors. This will make the passage to spectra easy: compose with the functor and realize. $\S 6.6 .1$ describes stratified maps obtained by realizing functors. 6.6 .2 gives a functor version of pullbacks over simplicial complexes, and 6.6.3 gives approximations of locally compact ANR filtered sets by simplicial complexes.

6.6.1 Realization of space-valued functors. Suppose $K$ is a simplicial complex, and consider $K$ as a category with objects the simplices and morphisms the inclusions $j_{i}: \partial_{i} \sigma \rightarrow \sigma$. Realization gives CW complex $|K|$.

Realization of complexes extends in a standard way to functors. Let $\hat{E}: K \rightarrow$ [spaces] be a contravariant functor. This means we get a space $\hat{E}(\sigma)$ for every simplex $\sigma \in K$, and if $\tau \subset \sigma$ then a map $\hat{E}(\sigma) \rightarrow \hat{E}(\tau)$. The realization is:

$$
|\hat{E}|=\coprod_{n}\left(\coprod_{\sigma^{n}} \hat{E}(\sigma) \times \Delta^{n}\right) / \sim
$$

where $\sim$ is given by: if $t \in \partial_{i} \Delta^{n}$ then $\left(x, j_{i}(t)\right) \in \hat{E}(\sigma) \times \Delta^{n}$ is identified with $\left(\hat{E}\left(j_{i}\right)(x), t\right) \in \hat{E}\left(\partial_{i} \sigma\right) \times \Delta^{n-1}$.

In these terms $|K|$ is the realization of the functor taking each simplex to a point. The natural transformation from an arbitrary functor $\hat{E}$ to the point functor gives a map of realizations $|\hat{E}| \rightarrow|K|$. The following lemma describes when this gives a homotopy stratified map: 
Lemma. Suppose $K$ is a simplicial set and $\hat{E}$ is a functor from $K$ to spaces. The map of realizations $|\hat{E}| \rightarrow|K|$ is homotopy stratified over a filtration of $|K|$ by subcomplexes if for any simplices $\tau \subset \sigma$ both in the same stratum of $K$ the map $\hat{E}(\sigma) \rightarrow \hat{E}(\tau)$ is a homotopy equivalence.

We often improve the output to be a stratified system of fibrations by applying stratified homotopy links, 6.5.2.

The point to be verified is that the restriction to a stratum is an approximate fibration. We can describe $|\hat{E}|$ as built up of iterated mapping cylinders, and the projection to $K$ is projection to iterated mapping cylinders of point maps. The structure on $K$ corresponds to describing a simplices $\Delta^{n}$ as the mapping cylinder of a map $S^{n-1} \rightarrow \partial \Delta^{n}$ union the mapping cylinder of $S^{n-1}$ to a point. The lemma now follows from the fact that iterated mapping cylinders of homotopy equivalences give approximate fibrations, c.f. [Hatcher 1].

The next result shows stratified systems of fibrations over simplicial sets are equivalent in a suitable sense to realizations.

6.6.2 Lemma (Functorialization). Suppose $p: E \rightarrow|K|$ is a stratified system of fibrations over a filtration of $|K|$ by subcomplexes. Then there is a functor $\hat{E}: K \rightarrow$ spaces so that the realization is homotopy stratified over the given filtration and there is a fiber homotopy equivalence of stratified systems of fibrations

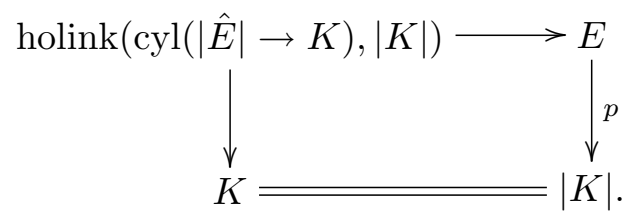

Recall that the homotopy link is the stratified system of fibrations canonically associated to the realization, which itself is only homotopy stratified.

Proof. We want a contravariant functor, and to get contravariance we use open stars. The open star of a simplex $\tau$ is the union of interiors of all simplices that contain $\tau$. Inclusions give this the structure of a contravariant functor of $\tau$ : if $\tau \subset \sigma$ then openstar $(\tau) \supset$ openstar $(\sigma)$. Define $\hat{E}(\sigma)$ to be the restriction of $E$ to openstar $(\sigma)$. This inherits a contravariant functor structure from the stars. The natural transformation to the point functor gives $|\hat{E}| \rightarrow|K|$ as above. The 
inverse image of a point $x$ is the total space of the pullback of $E$ over the open star of the simplex $\tau$ that contains $x$ in its interior.

There is a natural inclusion $E \subset|\hat{E}|$ defined by taking a point $e$ over $t \in \operatorname{int}(\sigma)$ to $(\{\sigma\}, e, t) \in\{\sigma\} \times(E \mid$ openstar $(\sigma)) \times \Delta^{n}$. This is a fiber map in the sense that it commutes with projections to $|K|$. Next we verify it is homotopy equivalences on fibers. Over a point $x$ in the interior of $\tau$ the map of fibers is the inclusion $E|x \subset E|$ openstar $(\tau)$. There is a (radial) deformation retraction of the open star to $x$ that preserves simplices, and therefore the stratification, until the last moment. Since $E$ is a fibration over strata this is covered by a deformation retraction of $E \mid$ openstar $(\tau)$ to $E \mid x$. This is a homotopy inverse for the inclusion, so the inclusion is a homotopy equivalence.

Now restrict to a stratum in the filtration of $K$. Over this $E$ is a fibration. The equivalence of point inverses just above and Lemma 6.6.1 show that $|\hat{E}|$ is an approximate fibration over this stratum. Thus the map $E \rightarrow|\hat{E}|$ is an approximate fiber homotopy equivalence. Applying the homotopy link construction to the approximate fibration turns it into a genuine fibration. A stratified homotopy equivalence of stratified systems of fibrations has a fiber homotopy inverse, and this provides the map of the lemma.

The final result of the section provides a bridge to the previous lemma from the general case.

6.6.3 Lemma (Simplicial approximation). Suppose $p: E \rightarrow X$ is a stratified system of fibrations over a locally compact metric ANR. Then for every $\epsilon>0$ there is a locally compact polyhedron $K$, a stratified systems of fibrations $q: F \rightarrow$ $K$ with simplicial stratification and a commutative diagram

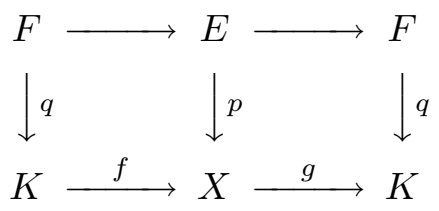

so that

(1) $f$ is proper and stratum-preserving and the fiber map over it induces homotopy equivalence of fibers;

(2) $g$ is filtration-preserving, and 
(3) the composition gf taking $p$ to itself is filtration-preserving fiber homotopic to the identity, by a homotopy of radius $<\epsilon$.

Note $g$ and the homotopy are not stratum-preserving. This can be arranged if $X$ is homotopy stratified, [Quinn 5]. The construction is sketched since it differs from previous versions only in that $X$ is not assumed finite dimensional, and the only application of this is to shorten theorem statements by two words.

Choose neighborhoods of the spaces $X_{n}$ in the filtration of $X$ for which there are almost-strict deformation retractions to $X_{n}$ covered by homotopies of $p$, as specified in the definition of stratified system of fibrations. Let $I^{\infty}$ denote the (compact) Hilbert cube, and choose a proper embedding $X \rightarrow[0, \infty) \times I^{P} \infty$. Since $X$ is an ANR there is a neighborhood that retracts to $X, r: U \rightarrow X$, and this is proper. We can choose $U$ and neighborhoods $U_{n}$ of $X_{n}$ so that

(1) there is a non-decreasing sequence $k_{j}$ so that $U$ and each $U_{n}$ intersected with $[0, j] \times I^{P} \infty$ is of the form $V \times I^{\infty-k_{j}}$ where $V$ is a closed codimension-0 submanifold of $[0, j] \times I^{k_{j}}$;

(2) $r$ takes $U_{n}$ into the neighborhood that deforms to $X_{n}$, and $U-U_{n}$ into $X-X_{n} ;$ and

(3) all the deformations, etc. are small compared to $\epsilon$.

Define a stratified system of fibrations over $U$ with filtration $U_{n}$ by pulling back $p$. Applying the neighborhood deformations in $X$ gives a small homotopy of $r$ to a stratum-preserving map covered by a map of systems of fibrations. This is the map $f$ of the Lemma. The composition of $f$ with the inclusion of $X$ is no longer the identity, but it has a small filtration-preserving homotopy to the identity.

The last step is to omit $I$ factors from $U$ to get a locally finite complex. Choose a monotone nonnegative sequence $i_{j}$ and consider the space $W=\cup_{n}(U \cap[j, j+$ 1] $\left.\times I^{k_{j}+i_{j}}\right)$. $W$ and each $W \cap U_{n}$ are unions of compact smooth manifolds so can be triangulated, giving $W$ the structure of a locally compact polyhedron with PL filtration. The restriction $r: W \rightarrow X$ is still proper, stratum-preserving, etc. There is an essentially canonical homotopy of $U$ into $W$ that is nearly the identity on $W$. In particular this homotopy takes the inclusion $X \rightarrow U$ to a map $q: X \rightarrow W$. The retraction to $X$ and this homotopy combine to give a homotopy of the composition $r q: X \rightarrow X$ to the identity. Since the $X_{n}$ are contained in the interiors of the $U_{n}$, if the homotopy is small enough this 
continues to be true during the homotopy. In other words $q$ and the homotopy are filtration-preserving. To complete the construction we observe that by choosing the sequence $i_{j}$ large enough we can arrange the homotopy of $U$ into $W$ to be arbitrarily small.

6.7 Spectral cosheaves and homology. In this section we review spaces and spectra over a space $X$, and homology of these. The discussion is the same as that in $\S 8$ of [Quinn 2] with a bit more detail and basepoints.

6.7.1 Spectra over $X$. A space over a space with basepoint $(X, *)$ is a map $p: E \rightarrow X$ so that $p^{-1}(*)$ is a single point (by definition the basepoint of $E$ ). A morphism over a map $X \rightarrow Y$ is a commutative diagram

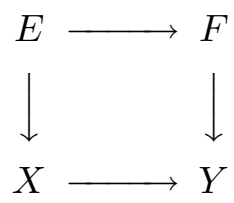

The map is required to preserve basepoints, but not complements of basepoints.

The usual notions of topology extend to spaces over other spaces, the only modifications being occasionally dividing out inverse images of basepoints [James 1]. A based space over $X$ is a space over $X$ with a section: $X \stackrel{s}{\rightarrow} E \stackrel{p}{\rightarrow} X$. If $\left(W, w_{0}\right)$ is a space with basepoint (not over anything) and $E$ is based over $X$ then the smash product over $X, E \wedge_{X} W$, is $E \times W / \sim$ where $\sim$ is the equivalence relation $\left(e, w_{0}\right) \sim(s p(e), w)$ for any $e \in E, w \in W$, and $(*, w) \sim *$. This defines another based space over $X$. For instance $E \wedge_{X} S^{1} \rightarrow X$ has point inverses suspensions of point inverses of $E \rightarrow X$. A spectrum over $X$ is a sequence of based spaces $E_{n}$ over $X$ and based morphisms $E_{n} \wedge_{X} S^{1} \rightarrow E_{n+1}$ over the identity of $X$.

The simplest example of a spectrum over $X$ is the product of $X$ and an ordinary spectrum. The ones we work with are elaborations of this:

6.7.2 Lemma. Suppose $K$ is a simplicial set and $\mathbb{E}: K \rightarrow$ spectra is a contravariant functor. Then realizations of the component spaces and maps of $\mathbb{E}$ define a spectrum over $|K|$.

In each degree $n \mathbb{E}$ has a space-with-basepoint valued functor $\mathbb{E}_{n}$ defined on $K$. Realizations of these provide a sequence of spaces over $|K|$. Structure maps 
in $\mathbb{E}$ provide natural transformations $\mathbb{E}_{n} \wedge S^{1} \rightarrow \mathbb{E}_{n+1}$. These induce morphisms of realizations $\left|\mathbb{E}_{n}\right| \wedge_{|K|} S^{1} \rightarrow\left|\mathbb{E}_{n+1}\right|$. These objects and morphisms constitute a spectrum over $|K|$.

6.7.3 Definition. A spectrum $\mathbb{E} \rightarrow X$ is a spectral cosheaf over $X$ if there is a filtration so that each $E_{n} \rightarrow X$ is a stratified system of fibrations over this filtration.

Most of the cosheaves we work with come from functors and a sharpened form of Lemma 6.7.2. In practice we mostly use 6.7.2, go directly to homology, and use properties of functors rather than properties of cosheaves. The exception is the iterated homology identity in 6.10 , where a spectral cosheaf is part of the input data.

6.7.4 Lemma. Suppose $K$ is a simplicial set with a filtration by subcomplexes, $\mathbb{E}: K \rightarrow$ spectra is a contravariant functor, and if $\sigma \subset \tau$ are in the same stratum then $\mathbb{E}(\tau) \rightarrow \mathbb{E}(\sigma)$ is an equivalence of spectra. Then realizations of the component spaces and maps of $\mathbb{E}$ give an "approximate" spectral cosheaf over $|K|$. Applying stratified homotopy links to mapping cylinders gives a spectral cosheaf.

Here "approximate" means the $E_{n} \rightarrow K$ are homotopy stratified (approximate fibrations over strata). This follows from Lemma 6.6.1, and the improvement using homotopy links comes from 6.5.2.

6.7.5 Homology. Suppose $X$ is a space with basepoint and $\mathbb{E}$ is a spectrum over $X$. The homology is the space defined by the direct limit

$$
H(X, * ; \mathbb{E})=\lim _{n \rightarrow \infty} \Omega^{n}\left(\mathbb{E}_{n} / X\right) .
$$

Some details: $\mathbb{E}_{n} / X$ is the space obtained by dividing out the image of the "basepoint" section $X \rightarrow \mathbb{E}_{n}$. Dividing by the section in a smash product over $X$ gives the ordinary smash product, so the structure map of the spectrum gives

$$
\left(\mathbb{E}_{n} / X\right) \wedge S^{1} \rightarrow \mathbb{E}_{n+1} / X
$$

which is just the structure of an ordinary spectrum (i.e. over a point). We now turn this into an $\Omega$-spectrum in the stantard manner: take the adjoint of the structure map and apply $\Omega^{n}$ to get

$$
\Omega^{n}\left(\mathbb{E}_{n} / X\right) \rightarrow \Omega^{n+1}\left(\mathbb{E}_{n+1} / X\right) .
$$


Finally take the direct limit of this system.

The homology space is in a natural way an $\Omega$-spectrum, denoted $\mathbb{H}(X ; \mathbb{E})$. Explicitly the $k^{\text {th }}$ space in the spectrum structure is obtained by changing the number of applications of $\Omega$ in the direct system:

$$
\mathbb{H}(X, * ; \mathbb{E})_{k}=\lim _{n \rightarrow \infty} \Omega^{n-k}\left(\mathbb{E}_{n} / X\right) .
$$

The homology groups are the homotopy groups of this spectrum: $H_{j}(X, * ; \mathbb{E})=$ $\pi_{j} \mathbb{H}(X, * ; \mathbb{E})$. Note that the homology and spectrum indexings go in opposite directions: $\pi_{j}\left(\mathbb{H}(X, * ; \mathbb{E})_{k}\right)=\pi_{j-k}\left(\mathbb{H}(X, * ; \mathbb{E})_{0}\right)=H_{j-k}(X, * ; \mathbb{E})$.

6.7.6 Locally finite homology. Locally finite homology is simply reduced homology of the 1-point compactification. If $\mathbb{E} \rightarrow X$ is a spectrum over a locally compact space then define a spectrum $\mathbb{E}^{+}$over $X^{+}$simply by adding a point to each $\mathbb{E}_{n}$ over $\infty$ and defining neighborhoods of the point to be inverse images of complements of compact sets in $X$. We then define

$$
\mathbb{H}^{l f}(X ; \mathbb{E})=\mathbb{H}\left(X^{+}, \infty ; \mathbb{E}^{+}\right) .
$$

See, however, the caution about 1-point compactifications in 6.3.2.

6.8 Functors and assembly. Suppose $J$ is a functor satisfying the hypotheses of the Characterization theorem, and let $\mathbb{J}: \mathcal{C}^{+} \rightarrow$ spectra be the spectrum-valued enhancement described in 6.5. In this section we construct the assembly map used in the theorem.

6.8.1 $\mathbb{J}$ homology. First suppose $K$ is a simplicial complex and $\hat{E}: K \rightarrow$ spaces is a contravariant functor. Think of a space as a map from the space to a point, and compose this $\mathbb{J}$ to get a functor $K \rightarrow$ spectra. More explicitly this means the functor $\sigma \mapsto \mathbb{J}(\mathrm{pt}, \hat{E}(\sigma) \rightarrow \mathrm{pt})$. Realization defines a spectrum over $|K|$. Denote this $|K|$-spectrum by $\mathbb{J}(\hat{p})$, where $\hat{p}$ is the projection $|\hat{E}| \rightarrow|K|$, or by $\mathbb{J}\left(\hat{p}^{-1}(\#)\right)$, where \# indicates this is to be thought of as a function of points in $|K|$. Neither notation is completely successful, so we usually spell out what they are supposed to mean in each context.

Homology spectra of these spectra are denoted $\mathbb{H}(|K| ; \mathbb{J}(p))$. In the locally compact case $\mathbb{H}^{l f}(|K| ; \mathbb{J}(p))=\mathbb{H}\left(|K|^{+}, * ; \mathbb{J}(p)^{+}\right)$. Finally, homology of general homotopy stratified $E \rightarrow X$ is defined to be the direct limit of homology of functor pullbacks over complexes (6.6.2), locally compact mapping properly to $X$ in the locally finite case. 
6.8.2 Proposition. Suppose $\mathbb{J}$ is a homotopy-invariant functor from spaces to spectra. Then the locally finite homology functor $J(p: E \rightarrow X)=\mathbb{H}^{l f}(X ; \mathbb{\mathbb { J }}(p))$ satisfies the homology axioms 6.1(1)-(4).

Note we are not using the full structure of $J$ of 6.1 , but just the "trivial base" case $E \rightarrow$ pt thought of as a functor of the space $E$. We will not give the proof here because it is long and technical and differs from the standard development of generalized homology [Whitehead 1] only in predictable ways. Perhaps the most subtle point is the union axiom (4) and the use of the full product topology. This is where the topology of the 1-point compactification enters crucially. If something is an infinite union then in the 1-point compactification most of the pieces are very close to the basepoint. This makes it possible for something compact (e.g. a sphere) to map nontrivially into all of them at once.

6.8.3 Assembly. In this section we assume $\mathbb{J}$ satisfies the hypotheses of the Characterization Theorem and from this construct the comparison map used in the theorem:

Lemma. There is a functorial map of spectra

$$
\mathbb{H}^{l f}(X ; \mathbb{J}(p)) \stackrel{A_{X}}{\longrightarrow} \mathbb{J}(X ; p)
$$

defined for $p: E \rightarrow X$ homotopically stratified over a locally compact ANR, and $A_{X}$ is the identity when $X$ is a point.

The "identity" in the last part of the statement refers to the equivalence

$$
\mathbb{H}(\mathrm{pt}, \mathbb{J}(E \rightarrow \mathrm{pt})) \simeq \mathbb{J}(E \rightarrow \mathrm{pt})
$$

We go through a series of reductions to get to the core construction. First it is sufficient to consider the realization of a functor over a locally compact complex, $p:|\hat{E}| \rightarrow|K|$ because the homology is the direct limit of homologies of these.

Second, homology is defined using loop spaces of quotients $\Omega^{n}\left(\mathbb{J}_{n}(p)^{+} /|K|\right)$, so it is sufficient to define maps on the quotients $\mathbb{J}_{n}(p)^{+} /|K| \rightarrow \mathbb{J}_{n}(|K|, p)$ and then take loops on these. Equivalently we define maps $\mathbb{J}_{n}(p) \rightarrow \mathbb{J}_{n}(|K|, p)$ that preserve "basepoints" in the sense that $|K|$ is taken to the basepoint of $\mathbb{J}_{n}(|K|, p)$.

Finally, $\mathbb{J}_{n}(p)$ is defined by realizing a functor and the right side is natural so it is sufficient to construct maps on pieces of the realization that fit together. For 
this we use the dual cones of the triangulation of $K$. Recall the dual cone $C_{\sigma}$ of a simplex $\sigma$ is the union of closed simplices in the first barycentric subdivision that intersect $\sigma$ but not $\partial \sigma . C_{\sigma}$ intersects $\sigma$ in the barycenter; it is the cone, with the barycenter as cone point, on the link of $\sigma$. Note $C_{\sigma}$ is contained in the open star of $\sigma$. To describe the data used in the construction of $A$ we need some notation.

Denote the projection by $q: \mathbb{J}_{n}(p) \rightarrow|K|$. Over a cone $C_{\sigma}$

$$
q^{-1}\left(C_{\sigma}\right)=\cup_{\alpha \in C_{\sigma}} \mathbb{J}_{n}(E \mid \text { openstar }(\bar{\alpha})) \times \alpha / \simeq .
$$

The union is over simplices in the subdivision lying in the cone, and if $\alpha$ is such a simplex then $\bar{\alpha}$ denotes the smallest simplex in the original triangulation containing $\alpha$. We have also used the abbreviated notation $\mathbb{J}_{n}(E \mid$ openstar $(\tau))$ for $\mathbb{J}_{n}(E \mid \operatorname{openstar}(\tau) \rightarrow \mathrm{pt})$. Since all the $\bar{\alpha}$ in the union contain $\sigma$ there are inclusions openstar $(\bar{\alpha}) \subset$ openstar $(\sigma)$. Projecting $q^{-1}\left(C_{\sigma}\right)$ to the $\mathbb{J}_{n}$ factors and composing with the inclusion defines a map $q^{-1}\left(C_{\sigma}\right) \rightarrow \mathbb{J}_{n}(E \mid$ openstar $(\sigma))$.

Denote the projection by $\hat{p}:|E| \rightarrow|K|$. The inverse image of a cone is a quotient of a union of products $E \mid \operatorname{openstar}(\bar{\alpha}) \times \alpha$ as above. Projecting to the $E$ factor gives a commutative diagram

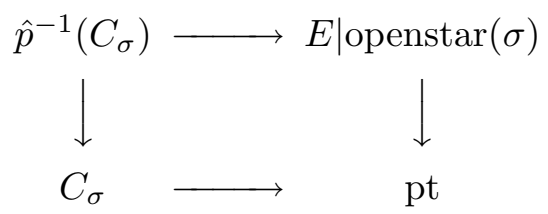

Applying $\mathbb{J}_{n}$ gives a morphism $\mathbb{J}_{n}\left(\hat{p}^{-1}\left(C_{\sigma}\right) \rightarrow C_{\sigma}\right) \longrightarrow \mathbb{J}_{n}(E \mid$ openstar $(\sigma))$.

In these terms the fragments of the assembly map are maps $A_{\sigma}$ and homotopies giving a homotopy-commutative diagram

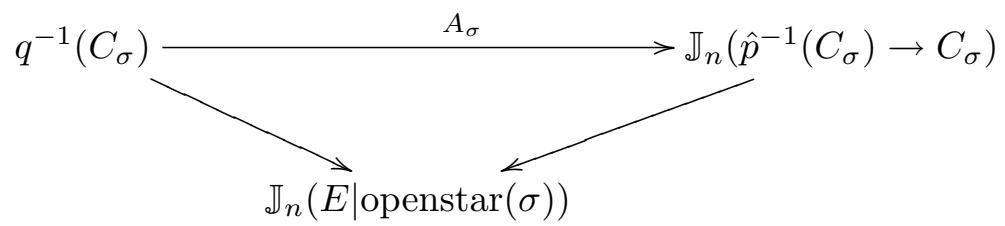

and these should be natural with respect to inclusions of cones.

These maps are constructed by induction on the dimension of the cone (so downward with respect to dimension of simplices, starting with maximal simplices). 0-dimensional cones have $A$ the identity. Suppose these are defined for 
cones of dimension $<k$ and let $\sigma$ be a simplex with $C_{\sigma}$ of dimension $k$. The data for the cones in $\partial C_{\sigma}$ fit together to give a map $A$ and a homotopy of the compositions into the lower right of the diagram

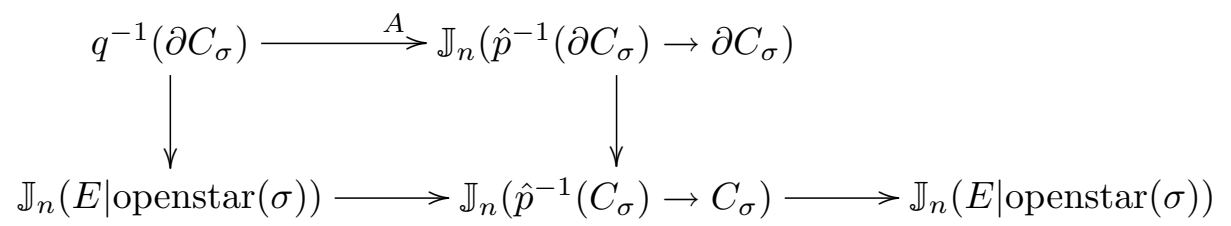

On the bottom row the first map includes $E \mid$ openstar $(\sigma) \rightarrow$ pt over the cone point, and the second map is induced by the projection of $\hat{p}^{-1}\left(C_{\sigma}\right)$ to $E \mid \operatorname{openstar}(\sigma)$. Both of these lower maps are homotopy equivalences by the homotopy axiom and the fact that the radial deformation retraction of the cone to the cone point is covered by a deformation of $\hat{p}^{-1}\left(C_{\sigma}\right)$. Thus the homotopy of compositions into the lower right space lifts to a homotopy of compostions into $\mathbb{J}_{n}\left(\hat{p}^{-1}\left(C_{\sigma}\right) \rightarrow C_{\sigma}\right)$. Now observe that $q^{-1}\left(C_{\sigma}\right)$ is the mapping cylinder of the left vertical map. The lifted homotopy defines a map on this mapping cylinder. This map is $A_{\sigma}$, and the homotopy in the lower right space gives the homotopy needed to complete the data.

In this fashion $A$ can be defined on all the $k$-dimensional cones, and by induction on the whole spectral functor realization. Note this $A$ is not completely canonical: there are choices in lifting the homotopy in the last step. However any two lifts are homotopic, any two homotopies between lifts are themselves homotopic, etc. This means for any practical purpose it can be considered natural and canonical.

6.9 Proof of the Characterization Theorem. This follows the traditional (Eilenberg-Steenrod) proof of uniqueness for ordinary homology, working up from points using excision and homotopy invariance.

Step 1. Theorem 6.1 holds for projections $F \times R^{n} \rightarrow R^{n}$.

Let $R_{+}^{n}$ denote the upper half space and $p$ the projection $F \times R_{+}^{n} \rightarrow R_{+}^{n}$. The inclusion $R^{n-1} \subset R_{+}^{n}$ gives excision sequences and a homotopy commutative 
diagram

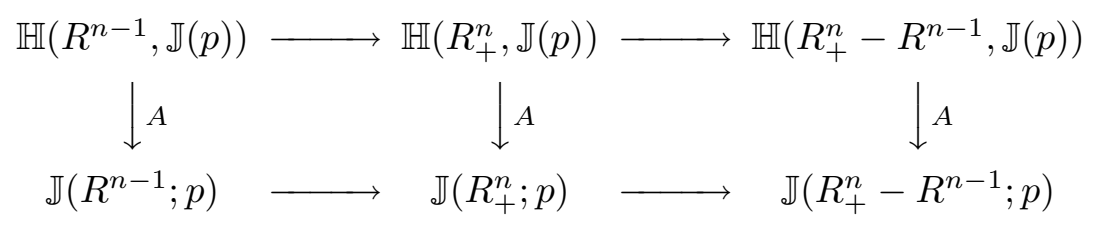

By excision the rows are homotopy fibrations. Thus if any two of the vertical maps are equivalences the third is also. The one-point compactification of the half space is contractible, so by homotopy invariance both functors applied to it give contractible spectra. Thus the center vertical map is trivially an equivalence. The space on the right, $R_{+}^{n}-R^{n-1}$, is homeomorphic to $R^{n}$. By construction the assembly map is an equivalence for a projection to a point $\left(=R^{0}\right)$, and this is the left vertical when $n=1$. The fibration property implies the right vertical, over $R^{1}$, is an equivalence. This argument shows inductively that assembly is an equivalence for all $n$.

Step 2. Theorem 6.1 holds for $p$ homotopy stratified over a finite complex filtered by subcomplexes.

Suppose $K$ is such a finite complex and let $K_{i}$ denote the $i$-skeleton. Then the inclusions $K_{i} \subset K_{i+1}$ give excision fibration sequences

$$
\mathbb{H}\left(K_{i} ;(p)\right) \rightarrow \mathbb{H}\left(K_{i+1} ;(p)\right) \rightarrow \mathbb{H}^{l f}\left(K_{i+1}-K_{i} ;(p)\right)
$$

and similarly for $\mathbb{J} . K_{i+1}-K_{i}$ is a (finite) union of copies of $R^{i+1}$ over which $p$ is constant, so Step 1 shows the assembly map is an equivalence for these terms. This applies to the right-hand term in all the fibration sequences, and the lefthand term when $i=0$. We can now proceed by induction as above. In a finite number of steps $i$ reaches the dimension of the complex so we conclude 6.1 holds for $K$.

Step 3. Theorem 6.1 holds for locally finite complexes and $p$ homotopy stratified over a filteration by subcomplexes.

For this we decompose a locally finite $|K|$ in a nice way. Define the closed star of $V \subset|K|$ to be the union of all closed simplices that intersect $V$. Note that if $V$ is closed then it is contained in the interior of its closed star.

Let $V_{0}$ consist of a vertex in each component of $|K|$, and inductively define $V_{n}$ to be the closed star of $V_{n-1}$. Define $A$ to be the union over $n$ of the closures of 
$V_{2 n}-V_{2 n-1}$. Define $B$ similarly as the union of closures of $V_{2 n+1}-V_{2 n}$. Note each of these pieces is a finite complex, and the unions are disjoint unions. We get:

(1) $|K|=A \cup B$;

(2) the components of $A$ and $B$ are finite subcomplexes of $|K|$

Since the theorem is true for finite complexes it is true for disjoint unions of finite complexes by the union axiom (for both $J$ and homology). Thus it is true for $A$, $B$, and $A \cap B$. Use excision twice, first with $A \cap B \subset B$ to conclude the theorem holds for $B-A \cap B=A \cup B-A$, and then with $A \subset A \cup B$ to conclude it holds for $A \cup B$.

Step 4. Theorem 6.1 holds in general.

Suppose $p: E \rightarrow X$ is homotopy stratified over the locally compact ANR $X$. According to the simplicial approximation and functor pullback lemmas 6.6.3 and 6.6.2 $p$ is a proper retract of the realization of a functor over the realization of a (locally finite) simplicial complex mapping properly to $X$. Since retracts of isomorphisms are isomorphisms the ANR case follows from the locally finite complex case.

This completes the proof of the Characterization Theorem.

6.10 The iterated homology identity. This is an equivalence of spectra so that application of the Atiyah-Hirzebruch type spectral sequence to its homotopy groups gives a Leray-Serre type spectral sequence for homology of a map.

This can be seen as a push-forward operation in the category of "spectra over spaces", see [May-Sigurdsson]. Given a spectrum over $X$ and a map $X \rightarrow Y$ define the push-forward to be the spectrum over $Y$ which over a point is the homology spectrum of the point-inverse in $X$. The homology of $X$ itself is then just the push-forward to a point. In these terms the iterated homology identity is functoriality of the push-forward. Given maps $X \rightarrow Y \rightarrow$ pt and a spectrum over $X$, pushing forward first to $Y$ and then to the point gives the same result as pushing forward directly to the point. This point of view leads to a much faster proof, but at the expense of an excursion into spectra over spaces and relating these to the functor-oriented constructions used here.

The compact-support version is stated in 6.10.1, the stratification hypothesis and its locally compact analog are discussed in 6.10.2, and the locally compact 
version of the theorem is given as 6.10.3. The remainder of the section contains proofs.

6.10.1 Theorem. (Iterated homology identity) Suppose $\mathbb{E} \stackrel{p}{\rightarrow} X \stackrel{f}{\rightarrow} Y$ satisfy:

(1) $X$ and $Y$ are filtered ANRs;

(2) $f$ is an "approximate stratified system of stratified fibrations" (see below) over the filtration of $Y$;

(3) $p$ is a spectral cosheaf over the filtration of $X$.

Then applying $\mathbb{H}(-; \mathbb{E})$ to point inverses in $X$ gives a spectral cosheaf $\mathbb{H}(f ; \mathbb{E})) \rightarrow$ $Y$, and there is a natural equivalence of spectra

$$
\mathbb{H}(Y, \mathbb{H}(f ; \mathbb{E}))) \stackrel{\simeq}{\longrightarrow} \mathbb{H}(X ; \mathbb{E})
$$

We discuss the stratification hypothesis.

6.10.2 Definition. A stratified fibration is a map $U \rightarrow V$ with a filtration on the total space that satisfies a stratum-preserving version of the lifting property for fibrations, see [Hughes 1, 2]. It follows that on each stratum the restriction $\left(U_{i}-U_{i-1}\right) \rightarrow V$ is a fibration, and in the case at hand this is nearly sufficient. We now extend this over strata in the base: $f: X \rightarrow Y$ is a stratified system of stratified fibrations if the restriction of the filtration of $X$ to the inverse of each stratum in $Y, f^{-1}\left(Y_{i}-Y_{i-1}\right) \rightarrow\left(Y_{i}-Y_{i-1}\right)$ is a stratified fibration, and $f$ satisfies a stratified version of the cofibration condition for the filtration of $Y$.

This is a lot of data. However it often will come naturally from the setting or can be arranged by general principles. Examples are:

(1) If $X$ and $Y$ are homotopy stratified sets then the projection $X \times Y \rightarrow Y$ is a stratified system of stratified fibrations.

(2) Suppose a finite group $G$ acts in a homotopy stratified way on a manifold $M$, suppose $H \subset G$ is a subgroup, and let $M^{(H)}$ denote the set of points fixed by some conjugate of $H . M^{(H)} / G$ is a homotopy stratified space with the orbit type filtration [Quinn 5]. The other strata of $M / G$ generally will contain $M^{(H)} / G$ in their closure. The stratified homotopy link [Hughes 1,2] provides a model for these strata in a neighborhood. It is a stratified system of stratified fibrations over $M^{(H)} / G$. 
(3) More generally if $Y \subset X$ is a pure subset (closed union of components of strata) in a homotopy stratified space then the stratified homotopy link is a stratified system of stratified fibrations over $Y$.

(4) If $K \rightarrow L$ is a proper PL map of polyhedra then there is a subdivison and a filtration of $K$ by subcomplexes so that $K \rightarrow L$ becomes a stratified system of stratified fibrations over the filtration of $L$ by skeleta.

(5) Generic smooth proper maps are stratified systems of fibrations with filtrations defined by stratifications of jet bundles and coincidence conditions.

We note that the map $f$ in the theorem is only assumed to be an approximate stratified system of stratified fibrations. This is important in geometric applications because the ones encountered are almost never genuine. The approximate versions of these definitions are spelled out in [Hughes 1, 2], but we can dodge the issue by using the characterization: a map is an approximate stratified system of stratified fibrations if the stratified homotopy link in the mapping cylinder is a genuine stratified system of stratified fibrations.

6.10.3 Locally finite iterated homology. The same result holds for locally finite homology if the map is proper:

Proposition. Suppose $E \rightarrow X \rightarrow Y$ are as in 6.10.1, $X, Y$ are locally compact and $f$ is proper. Then there is an equivalence

$$
\left.\mathbb{H}^{l f}(Y, \mathbb{H}(f ; \mathbb{E}))\right) \stackrel{\simeq}{\longrightarrow} \mathbb{H}^{l f}(X ; \mathbb{E}) .
$$

Note the point inverses of $f$ are compact so we do not have to specify locally finite or compact supports for the homology used in the cosheaf $\mathbb{H}(f ; \mathbb{E})$. There is also a version for nonproper maps and locally finite homology in the cosheaf. This requires a stratified proper homotopy local triviality hypothesis on the fibers of $f$ over strata in $Y$.

6.10.4 The map of spectra. We begin with the structure map of the spectrum over $X$ :

$$
S^{k} \wedge_{X} E_{n} \rightarrow E_{n+k} .
$$

A space over $X$ with a section has a partial quotient that gives a space over $Y$ with a section: given $X \rightarrow F \rightarrow X$ define $F / \simeq$ by identifying each of the images 
$f^{-1}(y) \rightarrow F$ to a point. Taking $y$ to this point defines the section $Y \rightarrow F / \simeq$. Applying this to the structure map gives spaces over $Y$ :

$$
S^{k} \wedge_{Y}\left(E_{n} / \simeq\right)=\left(S^{k} \wedge_{X} E_{n}\right) / \simeq \longrightarrow E_{n+k} / \simeq .
$$

This map is adjoint to a map to the loop space over $Y$ :

$$
E_{n} / \simeq \rightarrow \Omega_{Y}^{k}\left(E_{n+k} / \simeq\right) .
$$

Take the (homotopy) direct limit $k \rightarrow \infty$ and the right side becomes homology, by definition. This gives a map of spectra over $Y$

$$
E_{n} / \simeq \rightarrow \mathbb{H}(f ; \mathbb{E})_{n},
$$

To get the associated map of homology divide out $Y$ and apply $\Omega^{n}$ :

$$
\Omega^{n}\left(E_{n} / X\right)=\Omega^{n}\left(E_{n} / \simeq / Y\right) \rightarrow \Omega^{n}\left(\mathbb{H}(f ; \mathbb{E})_{n} / Y\right)
$$

Taking the limit $n \rightarrow \infty$ gives $\mathbb{H}(X ; \mathbb{E})$ on the left and $\left.\mathbb{H}\left(Y ; \mathbb{H}(f ; \mathbb{E})_{n} / Y\right)\right)$ on the right. This is the map.

We see that the theorem amounts to rearranging the direct limits in taking homology.

6.10.5 The proof. First note that the classical (constant-coefficient) case follows from the Characterization Theorem. Suppose $\mathbb{E}$ is a spectrum. Define a functor on maps $U \rightarrow Y$ by taking the $0^{\text {th }}$ space of the homology of $U$ and ignoring $Y: \mathbb{H}(U ; \mathbb{E})_{0}$. This satisfies the axioms. The theorem reconstructs the spectrum structure and when $U \rightarrow Y$ is homotopy stratified provides an assembly equivalence

$$
\mathbb{H}\left(Y ; \mathbb{H}\left(p^{-1}(\#) ; \mathbb{E}\right)\right) \stackrel{\simeq}{\longrightarrow} \mathbb{H}(U ; \mathbb{E}) .
$$

We now sketch the proof in the general case. First, by comparing excision fibrations and using the cofibration condition on the filtration of $Y$, reduce to the case where $Y$ has one stratum and $X \rightarrow Y$ is a stratified fibration.

Next by using ANR and compact support properties reduce to the case $Y$ a finite complex.

Again by comparing excision fibrations and working by induction on the number of cells we reduce to the relative case $Y=\left(D^{k}, S^{k-1}\right)$. Over a disk a stratified fibration is stratified homotopy equivalent to a product, so the disk case is a product $\mathbb{E} \times D^{n} \rightarrow W \times D^{n} \rightarrow D^{n}$, where $\mathbb{E} \rightarrow W$ is a spectral cosheaf.

Further excisions reduce this to the case $n=0$ where the equivalence is trivial. 


\section{REFERENCES}

Anderson, Douglas R., Munkholm, Hans Jørgen

1. Geometric modules and Quinn homology theory, K-Theory 7 (1993), 443-475, MR 95f:55003. Aravinda, C. S., Farrell, F. T.; Roushon, S. K.

1. Algebraic K-theory of pure braid groups, Asian J. Math. 4 (2000), 337-343, MR 2002a:19002. Bartels, Arthur; Farrell, Tom; Jones, Lowell; Reich, Holger

1. A foliated squeezing theorem for geometric modules, High-dimensional manifold topology, World Sci. Publ., River Edge, NJ,, 2003, pp. 1-21, MR2048714.

Bartels, A.; Reich, H.

1. On the Farrell-Jones Conjecture for higher algebraic K-theory, arXiv:math.AT/0308030.

Berkove, E.; Farrell, F. T.; Juan-Pineda, D.; Pearson, K.

1. The Farrell-Jones isomorphism conjecture for finite covolume hyperbolic actions and the algebraic K-theory of Bianchi groups, Trans. Amer. Math. Soc. 352 (2000), 5689- 5702, MR 2001b:57052.

Davis, James F.; Lück, Wolfgang

1. Spaces over a category and assembly maps in isomorphism conjectures in $K$ - and L-theory, $K$-Theory 15 (1998), 201- 252, MR 99m:55004.

Farrell, F. T., Jones, L. E.

1. Isomorphism conjectures in algebraic K-theory, J. Amer. Math. Soc. 6 (1993), 249- 297, MR 93h:57032.

2. Stable pseudoisotopy spaces of compact non-positively curved manifolds, J. Differential Geom. 34 (1991), 769-834, MR 93b:57020.

Hughes, Bruce

1. Stratified path spaces and fibrations, Proc. Roy. Soc. Edinburgh Sect. A 129 (1999), 351384, MR 2000d:57041.

2. Products and adjunctions of manifold stratified spaces, Topology Appl. 124 (2002), 47-67, MR 1926134.

3. The approximate tubular neighborhood theorem, Ann. of Math. 156 (2002), 867-889, MR 2004b:57031.

Igusa, Kiyoshi

1. Higher Franz-Reidemeister torsion, AMS/IP Studies in Advanced Mathematics 31 (2002), American Mathematical Society.

Igusa, Kiyoshi; and Klein, John

1. The Borel regulator map on pictures. I, II, K-Theory 7 (1993), American Mathematical Society, 201-267..

May, J. P.; Sigurdsson, J.

1. Mathematical Surveys and Monographs 132 (2006), American Mathematical Society, MR2271789.

Pedersen, Erik Kjær

1. Controlled algebraic K-theory, a survey, AMS Contemp. Math. 258 (2000), 351-368, MR 2001i:57034.

Pedersen, Erik Kjær; Yamasaki, Masayuki

1. Stability in controlled L-theory, preprint arXiv:math.GT/04022128.

Quinn, Frank

1. Ends of maps. I, Ann. of Math. (2) 110 (1979), 275-331, MR 82k:57009.

2. Ends of maps. II, Invent. Math. 68 (1982), 353-424, MR 84j:57011.

3. Ends of maps. III. Dimensions 4 and 5, J. Differential Geom. 17, 503-521, MR 84j:57012.

4. Ends of maps. IV. Controlled pseudoisotopy, Amer. J. Math. 108, 1139-1161, MR 88j:57014.

5. Homotopically stratified sets, J. Amer. Math. Soc. 1 (1988), 441-499, MR 89g:57050.

6. Geometric algebra, Springer Lecture Notes in Math. 1126 (1985), 182-198, MR 86m:57023. 
7. Algebraic K-theory of poly-(finite or cyclic) groups, Bull. Amer. Math. Soc. (N.S.) 12 (1985), 221-226, MR 86e:18015.

8. Hyperelementary assembly for K-theory over virtually abelian groups, arXiv:math.KT/0509294 preprint 2005.

Roushon, S. K.

1. K-theory of virtually poly-surface groups, Algebr. Geom. Topol. 3 (2003), 103- 116. Suslin, A. A.

1. On the equivalence of $K$-theories, Comm. Algebra 9 (1981), 1559-1566, MR 82m:18008.

Volodin, I. A.

1. Algebraic K-theory, Uspehi Mat. Nauk 27 (1972), 207-208., MR0393191.

Wagoner, J.

1. Equivalence of algebraic K-theories, J. Pure Appl. Algebra 11 (1977/78), 245-269., MR0472796.

\section{Frank Quinn}

Math, Virginia Tech

Blacksburg, VA 24061-0123

USA

E-mail: quinn@math.vt.ed 